J. Inst. Math. Jussieu (2023), 22(1), 71-144

doi:10.1017/S1474748021000074 C The Author(s), 2021. Published by Cambridge University

Press. This is an Open Access article, distributed under the terms of the Creative Commons

Attribution licence (https://creativecommons.org/licenses/by/4.0/), which permits unrestricted re-use, distribution and reproduction, provided the original work is properly cited.

\title{
RECIPROCITY SHEAVES AND THEIR RAMIFICATION FILTRATIONS
}

\author{
KAY RÜLLING (D) ${ }^{1}$ AND SHUJI SAITO ${ }^{2}$ \\ ${ }^{1}$ Bergische Universität Wuppertal, Gaußstr 20, 42119 Wuppertal, Germany and \\ Technische Universität München, Boltzmannstr. 3, 85748 Garching, Germany \\ (ruelling@uni-wuppertal.de) \\ ${ }^{2}$ Graduate School of Mathematical Sciences, University of Tokyo, 3-8-1 Komaba, Tokyo, \\ 153-8914, Japan \\ (sshuji@msb.biglobe.ne.jp)
}

(Received 24 April 2020; revised 3 February 2021; accepted 3 February 2021; first published online 19 March 2021)

\begin{abstract}
We define a motivic conductor for any presheaf with transfers $F$ using the categorical framework developed for the theory of motives with modulus by Kahn, Miyazaki, Saito and Yamazaki. If $F$ is a reciprocity sheaf, this conductor yields an increasing and exhaustive filtration on $F(L)$, where $L$ is any henselian discrete valuation field of geometric type over the perfect ground field. We show that if $F$ is a smooth group scheme, then the motivic conductor extends the Rosenlicht-Serre conductor; if $F$ assigns to $X$ the group of finite characters on the abelianised étale fundamental group of $X$, then the motivic conductor agrees with the Artin conductor defined by Kato and Matsuda; and if $F$ assigns to $X$ the group of integrable rank 1 connections (in characteristic 0 ), then it agrees with the irregularity. We also show that this machinery gives rise to a conductor for torsors under finite flat group schemes over the base field, which we believe to be new. We introduce a general notion of conductors on presheaves with transfers and show that on a reciprocity sheaf, the motivic conductor is minimal and any conductor which is defined only for henselian discrete valuation fields of geometric type with perfect residue field can be uniquely extended to all such fields without any restriction on the residue field. For example, the Kato-Matsuda Artin conductor is characterised as the canonical extension of the classical Artin conductor defined in the case of a perfect residue field.
\end{abstract}

Keywords and phrases: Reciprocity sheaf, conductor, ramification

2020 Mathematics subject classification: 14C25, 14F42, $19 \mathrm{~F} 15$

1 Introduction

1.1 Conventions .................................................. 76

$\begin{array}{ll}\text { Part 1. The general theory } & \mathbf{7 7}\end{array}$

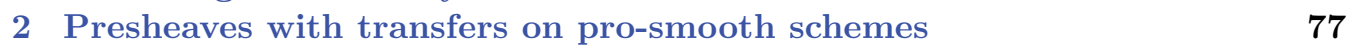

3 Review of reciprocity sheaves $\quad 80$

4 Conductors for presheaves with transfers $\quad \mathbf{8 4}$

4.1 Semicontinuous conductors .................................. 93

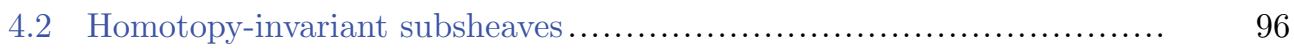

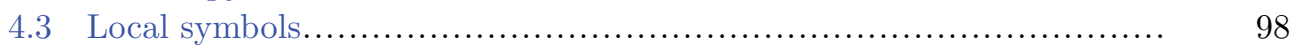


$\begin{array}{ll}\text { Part 2. Applications } & 103\end{array}$

5 Algebraic groups and the local symbol $\quad 103$

6 Differential forms and irregularity of rank 1 connections 105

6.1 Kähler differentials ............................................. 105

6.2 Rank 1 connections and irregularity .............................. 109

7 Witt vectors of finite length $\quad 112$

8 Lisse sheaves of rank 1 and the Artin conductor $\quad \mathbf{1 2 9}$

8.1 The case of finite monodromy .................................. 129

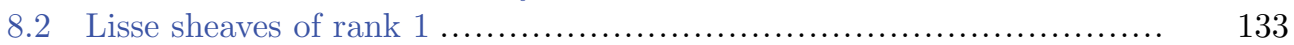

9 Torsors under finite group schemes over a perfect field 135

$\begin{array}{lr}\text { References } & 142\end{array}$

\section{Introduction}

Fix a perfect field $k$ and let $\mathbf{S m}$ be the category of separated smooth $k$-schemes. Let Cor be the category of finite correspondences: it has the same objects as $\mathbf{S m}$, and morphisms in Cor are finite correspondences (see Section 2.1 for a precise definition). Let PST be the category of additive presheaves of abelian groups on Cor, called presheaves with transfers. In this paper we give a construction which associates to each $F \in$ PST a collection of functions

$$
c^{F}=\left\{c_{L}^{F}: F(L) \rightarrow \mathbb{N} \cup\{\infty\}\right\}_{L \in \Phi},
$$

where $\mathbb{N}$ is the set of nonnegative integers, $\Phi$ is the collection of henselian discrete valuation fields which are the fraction fields of the henselisation $\mathcal{O}_{X, x}^{h}$ of $X \in \mathbf{S m}$ at points $x$ of codimension 1 in $X$ and

$$
F(L)=\underset{V}{\lim } F\left(V-D_{x}\right),
$$

where $V \rightarrow X$ ranges over étale neighbourhoods of $x$ and $D_{x}$ is the closure of $x$ in $V$. We call $c^{F}$ the motivic conductor for $F$. Our main aim is to convince the reader that our construction deserves such pretentious terminology. Indeed, it gives a unified way to understand different conductors, such as the Artin conductor of a character of the abelian fundamental group $\pi_{1}^{\mathrm{ab}}(X)$ with $X \in \mathbf{S m}$ along a boundary of $X$, the RosenlichtSerre conductor of a morphism from a curve to a commutative algebraic $k$-group and the irregularity of a line bundle with connections on $X \in \mathbf{S m}$ along a boundary of $X$. It also gives rise to a new conductor for $G$-torsors with $G$ a finite flat $k$-group scheme. The latter conductor specialises to the classical Artin conductor when $G$ is constant.

Our construction of the motivic conductors is rather simple once we have the new categorical framework introduced in [24, 25] at our disposal (see equation (1.0.1)). The main aim of those works is to develop a theory of motives with modulus generalising Voevodsky's theory of motives in order to capture non- $\mathbf{A}^{1}$-invariant phenomena and objects. The basic principle is that the category Cor should be replaced by the larger

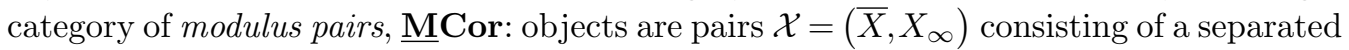
$k$-scheme of finite type $\bar{X}$ and an effective (possibly empty) Cartier divisor $X_{\infty}$ on it such that the complement $\bar{X} \backslash X_{\infty}$ is smooth. Morphisms are given by finite correspondences 
between the smooth complements satisfying certain admissibility conditions (see Section 3

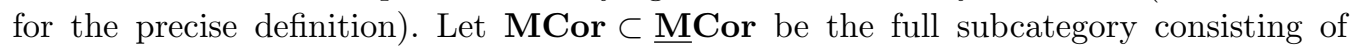
objects $\left(\bar{X}, X_{\infty}\right)$ with $\bar{X}$ proper over $k$. We then define MPST (resp., MPST) as the category of additive presheaves of abelian groups on MCor (resp., MCor). We have a functor

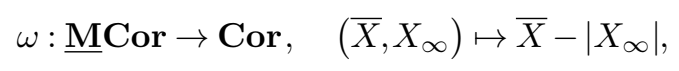

and two pairs of adjunctions

$$
\operatorname{MPST} \underset{\tau_{!}}{\stackrel{\tau^{*}}{\longrightarrow}} \text { MPST, MPST } \underset{\underline{\omega_{!}}}{\stackrel{\omega^{*}}{\longrightarrow}} \text { PST, }
$$

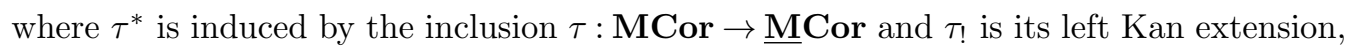
and $\omega^{*}$ is induced by $\omega$ and $\omega_{1}$ is its left Kan extension (see Section 3.3 for more concrete descriptions of these functors). A basic notion is $\bar{\square}$-invariance, where $\bar{\square}=\left(\mathbf{P}^{1}, \infty\right) \in$ MCor $-F \in$ MPST is called $\bar{\square}$-invariant if $F(\mathcal{X}) \simeq F(\mathcal{X} \otimes \bar{\square}$ ) for all $\mathcal{X} \in$ MCor (see Section 3.1 for the tensor product $\otimes$ in $\mathbf{M C o r}$ ). It is an analogue of the $\mathbf{A}^{1}$-invariance ${ }^{1}$ exploited by Voevodsky in his theory of motives. We write CI for the full subcategory of MPST consisting of $\bar{\square}$-invariant objects. We know [27, Lemma 2.1.7] that the inclusion CI $\rightarrow$ MPST admits a right adjoint $h_{\bar{\square}}^{0}$ which associates to $F \in$ MPST the maximal $\square$-invariant subobject of $F$. We define the functor

$$
\omega^{\mathrm{CI}}: \text { PST } \stackrel{\omega^{*}}{\longrightarrow} \text { MPST } \stackrel{h_{\square}^{0}}{\longrightarrow} \text { CI, }
$$

and write $\tilde{F}=\tau ! \omega^{\mathbf{C I}} F \in \underline{\mathbf{M P S T}}$, for $F \in \mathbf{P S T}$. Then the motivic conductor $c^{F}$ for $F \in$ PST is defined by

$$
c_{L}^{F}(a)=\min \left\{n \mid a \in \tilde{F}\left(\mathcal{O}_{L}, \mathfrak{m}_{L}^{-n}\right)\right\}, \quad \text { for } a \in F(L) .
$$

Here, for $G \in \underline{\text { MPST, } L=\operatorname{Frac}}\left(\mathcal{O}_{X, x}^{h}\right) \in \Phi$ and $n \in \mathbb{Z}_{\geq 1}$, we put

$$
G\left(\mathcal{O}_{L}, \mathfrak{m}_{L}^{-n}\right)=\underset{V}{\lim } G\left(V, n D_{x}\right),
$$

where $V \rightarrow X$ ranges over étale neighbourhoods of $x$ and $D_{x}$ is the closure of $x$ in $V$ and $n D_{x}$ is its $n$th thickening in $V$. By convention,

$$
G\left(\mathcal{O}_{L}, \mathfrak{m}_{L}^{-n}\right)=G\left(\mathcal{O}_{L}\right)=\underset{V}{\lim } G(V), \quad \text { for } n=0 .
$$

For $G=\tilde{F}$ there are natural inclusions $\tilde{F}\left(\mathcal{O}_{L}, \mathfrak{m}_{L}^{-n}\right) \hookrightarrow F(L)$, which are used to define equation (1.0.1). It turns out that $\left\{\tilde{F}\left(\mathcal{O}_{L}, \mathfrak{m}_{L}^{-n}\right)\right\}_{n \in \mathbb{Z}_{\geq 0}}$ induces an increasing filtration on $F(L)$ which is exhaustive if $F \in \mathbf{R S C}$. Here RSC is the full subcategory of PST consisting of the objects belonging to the essential image of $\mathbf{C I}$ under $\omega_{!}$. Objects of RSC are called reciprocity presheaves and play a key role in this note. We know (see [27, Cor 2.3.4]) that RSC contains all $\mathbf{A}^{1}$-invariant objects in PST. Moreover, it contains

\footnotetext{
${ }^{1}$ Recall that $F \in \mathbf{P S T}$ is $\mathbf{A}^{1}$-invariant if $F(X) \simeq F\left(X \times \mathbf{A}^{1}\right)$ for all $X \in \mathbf{S m}$.
} 
many interesting objects $F$ which are not $\mathbf{A}^{1}$-invariant. In this paper we consider in particular the following examples (where $X$ runs over objects of $\mathbf{S m}$ ):

(i) $F(X)=\operatorname{Hom}_{\mathbf{S m}}(X, \Gamma)$, where $\Gamma$ is a smooth commutative algebraic $k$-group which may have nontrivial unipotent part (for example, $\Gamma=\mathbf{G}_{a}$ ).

(ii) $F(X)=H_{\text {ét }}^{1}(X, \mathbb{Q} / \mathbb{Z})=\operatorname{Hom}_{\text {cont }}\left(\pi_{1}(X)^{\mathrm{ab}}, \mathbb{Q} / \mathbb{Z}\right)$.

(iii) $F(X)=\operatorname{Conn}^{1}(X)$ (resp., Conn $\left._{\text {int }}^{1}(X)\right)$ the group of isomorphism classes of (resp., integrable) rank 1 connections on $X$. Here we assume $\operatorname{ch}(k)=0$.

(iv) $F(X)=H_{\mathrm{fppf}}^{1}(X, \Gamma)$, where $\Gamma$ is a finite flat $k$-group.

We prove the following (see Theorems 5.2, 7.20, 8.8 and 6.11 for the precise statements):

\section{Theorem 1.}

(1) In case (i), $c_{L}^{F}$ agrees with the Rosenlicht-Serre conductor [4'7] if $L$ has perfect residue field. If $\operatorname{ch}(k)=p$ is positive and $F=W_{n}$ is the group scheme of p-typical Witt vectors of length $n$, then $c_{L}^{F}$ agrees with a conductor defined by Kato and Russell in [32] for any $L$.

(2) In case (ii), $c_{L}^{F}$ agrees with the Artin conductor Art $_{L}$ of Kato and Matsuda (see Section 7.1). ${ }^{2}$

(3) In case (iii), $c^{F}$ agrees with the irregularity of connections.

As far as we know, the motivic conductor $c^{F}$ in case (iv) is new, and we give an explicit description only in the case where the infinitesimal unipotent part of $G$ is $\alpha_{p}$, where $p=\operatorname{ch}(k)$ (see Theorem 9.12).

An amusing application of the motivic conductor $c^{F}$ is to give an explicit description of the maximal $\mathbf{A}^{1}$-invariant part of $F$ : let $\mathbf{H I} \subset \mathbf{P S T}$ be the full subcategory of $\mathbf{A}^{1}$ invariant objects. The inclusion $\mathbf{H I} \rightarrow \mathbf{P S T}$ admits a right adjoint $h_{\mathbf{A}^{1}}^{0}$ which associates to $F \in \mathbf{P S T}$ the maximal $\mathbf{A}^{1}$-invariant subobject of $F$ (see Section 4.30 for an explicit description of $\left.h_{\mathbf{A}^{1}}^{0}\right)$. Let $\mathbf{N S T} \subset \mathbf{P S T}$ be the full subcategory of Nisnevich sheaves - that is, those objects $F \in \mathbf{P S T}$ whose restrictions to $\mathbf{S m} \subset \mathbf{C o r}$ are sheaves with respect to the Nisnevich topology.

Theorem 2. For $F \in \mathbf{R S C} \cap \mathbf{N S T}$ and $X \in \mathbf{S m}$, we have

$$
h_{\mathbf{A}^{1}}^{0}(F)(X)=\bigcap_{\rho}\left\{a \in F(X) \mid c^{F}\left(\rho^{*} a\right) \leq 1\right\},
$$

where $\rho$ ranges over all morphisms $\operatorname{Spec} L \rightarrow X$ with $L \in \Phi$.

When $F=H_{\text {ét }}^{1}\left(-, \mathbb{Q} / \mathbb{Z}\right.$ ) from case (ii) (resp., $F=$ Conn $_{\text {int }}^{1}$ from case (iii)), Theorem 2 asserts that the maximal $\mathbf{A}^{1}$-invariant part of $F$ is precisely the subsheaf of tame characters (resp., regular singular connections).

\footnotetext{
${ }^{2}$ It coincides with the classical Artin conductor if $L$ has perfect residue field.
} 
In what follows, we fix $F \in \mathbf{R S C} \cap \mathbf{N S T}$ and introduce a class of collections of functions

$$
c=\left\{c_{L}: F(L) \rightarrow \mathbb{N}\right\}_{L \in \Phi}
$$

which may be called conductors for $F$. Let $\operatorname{Func}_{\Phi}(F, \mathbb{N})$ be the partially ordered set consisting of collections of functions with partial order given by $c \leq c^{\prime}$, if $c_{L}(a) \leq c_{L}^{\prime}(a)$ for all $L \in \Phi$ and $a \in F(L)$. Let $\mathbf{C I}(F)$ be the partially ordered set consisting of subobjects $G$ of $\omega^{\mathbf{C I}} F$ such that the induced maps $\omega_{!} G \rightarrow \omega_{!} \omega^{\mathbf{C I}} F$ are isomorphisms and with partial order given by inclusion. Then every $G \in \mathbf{C I}(F)$ gives rise to an exhaustive increasing filtration $\left\{\tau_{!} G\left(\mathcal{O}_{L}, \mathfrak{m}_{L}^{-n}\right)\right\}_{n \geq 0}$ on $F(L)$, and we define $c^{G} \in \operatorname{Func}_{\Phi}(F, \mathbb{N})$ by

$$
c_{L}^{G}(a)=\min \left\{n \mid a \in \tau_{!} G\left(\mathcal{O}_{L}, \mathfrak{m}_{L}^{-n}\right)\right\}, \quad \text { for } a \in F(L) .
$$

By definition, the motivic conductor $c^{F}$ of $F$ is $c^{\omega^{\mathbf{C I}} F}$ and $c^{F} \leq c^{G}$, for all $G \in \mathbf{C I}(F)$. Now a question is whether there is a simple characterisation of the poset $\left\{c^{G} \mid G \in \mathbf{C I}(F)\right\}$

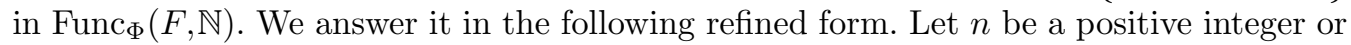
$\infty$. Let $\Phi_{\leq n} \subset \Phi$ be the collection of such $L$ that $\operatorname{trdeg}_{k}(L) \leq n$. (Note that in positive characteristic, $\Phi_{\leq 1}$ consists precisely of those $L \in \Phi$ that have a perfect residue field.) Let Func $_{\Phi}(F, \mathbb{N})_{\leq n}$ be the poset consiting of collections of functions

$$
c=\left\{c_{L}: F(L) \rightarrow \mathbb{N}\right\}_{L \in \Phi_{\leq n}}
$$

with partial order defined in the same manner as $\operatorname{Func}_{\Phi}(F, \mathbb{N})$. There is an obvious restriction functor

$$
\operatorname{Func}_{\Phi}(F, \mathbb{N}) \rightarrow \operatorname{Func}_{\Phi}(F, \mathbb{N})_{\leq n}, \quad c \mapsto c^{\leq n}
$$

We then introduce the six axioms $(c 1)-(c 6)$ for $\operatorname{Func}_{\Phi}(F, \mathbb{N})_{\leq n}$ (compare Definitions 4.3 and 4.22) and call those elements satisfying the axioms semicontinuous conductors of level $n$. Let $\operatorname{Cond}(F)_{\leq n}^{s c}$ be the subposet of $\operatorname{Func}_{\Phi}(F, \mathbb{N})_{\leq n}$ consisting of such objects. Write $\operatorname{Cond}(F)^{s c}$ for $\operatorname{Cond}(F)_{\leq n}^{s c}$ with $n=\infty .{ }^{3}$ For example, for $F=H_{\text {ét }}^{1}(-, \mathbb{Q} / \mathbb{Z})$ from case ii, the classical Artin conductor $\left\{\operatorname{Art}_{L}\right\}_{L \in \Phi_{\leq 1}}$ is an element of $\operatorname{Cond}(F)_{\leq 1}^{s c}$ and the Kato-Matsuda conductor $\left\{\operatorname{Art}_{L}\right\}_{L \in \Phi}$ is an element of $\operatorname{Cond}(F)^{s c}$. We show the following (see Theorem 4.25):

\section{Theorem 3.}

(1) $c^{G} \in \operatorname{Cond}(F)^{s c}$ for every $G \in \mathbf{C I}(F)$.

(2) There exists an order-reversing map

$$
\operatorname{Cond}(F)_{\leq n}^{s c} \rightarrow \mathbf{C I}(F), \quad c \mapsto \hat{F}_{c}
$$

such that $c=\left(c^{\hat{F}_{c}}\right)^{\leq n}$. For $\mathcal{X}=\left(\bar{X}, X_{\infty}\right) \in \operatorname{MCor}$ with $X=\bar{X}-\left|X_{\infty}\right|$, we have

$$
\hat{F}_{c}(\mathcal{X})=\left\{a \in F(X) \mid c_{\bar{X}}(a) \leq X_{\infty}\right\}
$$

${ }^{3}$ There is one axiom, (c4), which is not preserved by functor (1.0.2). So it does not induce $\operatorname{Cond}(F)^{s c} \rightarrow \operatorname{Cond}(F)_{\leq n}^{s c}$. 
where $c_{\bar{X}}(a) \leq X_{\infty}$ means that for any $L \in \Phi_{\leq n}$ and any morphism $\rho: \operatorname{Spec} \mathcal{O}_{L} \rightarrow \bar{X}$ such that $\rho(\operatorname{Spec} L) \in X, c_{L}\left(\rho^{*} a\right)$ is not more than the multiplicity of the pullback of $X_{\infty}$ along $\rho$.

As a consequence, we obtain the following (see Theorem 4.25(4)):

Corollary 1. There exists a unique map

$$
\operatorname{Cond}(F)_{\leq n}^{s c} \rightarrow \operatorname{Cond}(F)^{s c}, \quad c \mapsto c^{\infty},
$$

such that $\hat{F}_{c}=\hat{F}_{c^{\infty}}$ and $c=\left(c^{\infty}\right) \leq n$.

We call $c^{\infty}$ the canonical extension of $c$. For example, the Kato-Matsuda Artin conductor is the canonical extension of the classical Artin conductor. We say $F$ has level $n$ if $\left(c^{F}\right)^{\leq n} \in \operatorname{Cond}(F)_{\leq n}^{s c}$; in this case, $c^{F}$ is the canonical extension of $\left(c^{F}\right)^{\leq n}$, by Theorem 4.25(5). We show that $F=H_{\text {ét }}^{1}(-, \mathbb{Q} / \mathbb{Z})$ in case ii is of level 1 (see Theorem 8.8), $F=$ Conn $^{1}$ (resp., $F=$ Conn $_{\text {int }}^{1}$ ) from case (iii) is of level 2 (resp., 1) - see Theorem 6.11 - and $F=H_{\text {fppf }}^{1}(-, \Gamma)$ from case (iv) is of level 1 if the infinitesimal unipotent part of $\Gamma$ is trivial and is of level 2 otherwise (see Theorem 9.12).

In Section 2 we explain how to extend a presheaf with transfers to the category of regular schemes over $k$ which are pro-smooth; this is well known, and we include it only for lack of reference. In Section 3 we recall the necessary constructions and results from the theory of motives with modulus as developed in [24, 25, 27, 26, 45]. Then we introduce in Section 4 the notion of (semicontinuous) conductors and prove Theorems 3 and 2. We close the section with a discussion of the relation between the motivic conductor of a reciprocity sheaf and certain vanishing properties of its associated symbol. This is needed in order to prove in the later sections that a certain conductor is equal to the motivic one, the main point being Corollary 4.40. In the second part we consider various conductors which are mostly classical and show that they are motivic in our sense. Kähler differentials and rank 1 connections are considered in Section 6 , where $\operatorname{ch}(k)=0$. In the following sections we assume $\operatorname{ch}(k)=p>0$. In Section 7 we show that one of the conductors defined by Kato and Russell for $W_{n}$ is motivic. We use this in Section 8 to show that the KatoMatsuda conductor for characters is motivic, which yields also a description of the motivic conductor for lisse $\overline{\mathbb{Q}}_{\ell}$-sheaves of rank 1 . Finally, in Section 9 we define and investigate a conductor for torsors under finite flat $k$-groups, which we believe to be new. The general pattern of these computations is always the same: first we show that the collection $c=\left\{c_{L}\right\}$ defined in the various situations defines a semicontinuous conductor (of a certain level) in the sense of Definitions 4.3 and 4.14, then we do a symbol computation to show that this conductor is actually motivic. Note, however, that the actual computations in the various cases differ quite a bit.

\subsection{Conventions}

We work over a perfect field $k$. If $K / k$ is a field extension, then by a $K$-scheme we will always mean a scheme which is separated and of finite type over $K$. In contrast, the phrase scheme over $K$ refers to any scheme morphism $X \rightarrow \operatorname{Spec} K$. By a smooth $K$-scheme we 
mean a $K$-scheme which is smooth over $K$. We denote by $\mathbf{S m}_{K}$ the category of such schemes and set $\mathbf{S m}=\mathbf{S m}_{k}$. For $k$-schemes $X$ and $Y$ we write $X \times Y$ instead of $X \times{ }_{k} Y$. For any scheme $X$ we denote by $X^{(i)}$ the set of $i$-codimensional points of $X$.

\section{Part 1. The general theory}

\section{Presheaves with transfers on pro-smooth schemes}

The material in this section is well known; we give some details for lack of reference.

2.1. Denote by Cor the category of finite correspondences of Suslin and Voevodsky. Recall that the objects are the smooth $k$-schemes, and morphisms are given by correspondences - that is, $\operatorname{Cor}(X, Y)$ is the free abelian group generated by prime correspondences, the integral closed subschemes $V \subset X \times Y$ which are finite and surjective over a connected component of $X$. Given two prime correspondences $V \in \operatorname{Cor}(X, Y)$ and $W \in \operatorname{Cor}(Y, Z)$, their composition is given by the intersection product (see, for example, $[46, \mathrm{~V}, \mathrm{C}])$

$$
W \circ V=p_{13 *}\left(p_{12}^{*} V \cdot p_{23}^{*} W\right)
$$

where $p_{i j}$ denotes the projection from $X \times Y \times Z$ to the factor $(i, j)$.

Denote by ProCor the pro-category of Cor - that is, objects are functors $I^{\mathrm{o}} \rightarrow \mathbf{S m}$, $i \mapsto X_{i}$, where $I$ is a filtered essentially small category, and the morphisms between two pro-objects $\left(X_{i}\right)_{i \in I}$ and $\left(Y_{j}\right)_{j \in J}$ are given by

$$
\operatorname{ProCor}\left(\left(X_{i}\right),\left(Y_{j}\right)\right)={\underset{j}{j \in J}}_{i \in I} \underset{i \in I}{\lim } \operatorname{Cor}\left(X_{i}, Y_{j}\right)
$$

Definition 2.2. We define the category Cor $^{\text {pro }}$ as follows: the objects are the Noetherian regular schemes over $k$ of the form

$$
X=\lim _{i \in I} X_{i}
$$

where $\left(X_{i}\right)_{i \in I}$ is a projective system of smooth $k$-schemes indexed by a partially ordered set and with affine transition maps $X_{i} \rightarrow X_{j}, i \geq j$. If $X$ and $Y$ are two objects in Cor $^{\text {pro }}$, then $\operatorname{Cor}^{\text {pro }}(X, Y)=\operatorname{Cor}(X, Y)$ is the free abelian group generated by prime correspondences in the sense of Section 2.1. The composition is defined in the same way as in the case of Cor. (Note that this still makes sense by [46, V, B, 3., Théorème 1].)

\section{Remark 2.3.}

(1) All objects in Cor $^{\text {pro }}$ are separated, Noetherian and regular schemes over $k$. Any affine, Noetherian and regular scheme over $k$ defines an object in Cor ${ }^{\text {pro }}$, by [41, (1.8) Theorem] and [1, Exp I, Proposition 8.1.6].

(2) Note that for $X, Y \in \mathbf{C o r}^{\text {pro }}$, the cartesian product $X \times Y$ does not need to be Noetherian; but if $Y \in \mathbf{S m}$ and $X \in \mathbf{C o r}^{\text {pro }}$, then $X \times Y \in \mathbf{C o r}^{\text {pro }}$.

Lemma 2.4. Let $A$ be a k-algebra which is Noetherian, regular and a directed limit $A=\lim _{i \in I} A_{i}$, where the $A_{i}$ are smooth and of finite type over $k$ and the transition maps $A_{i} \rightarrow \vec{A}_{j}, j \geq i$ are flat. Let $X$ be a regular quasi-projective $A$-scheme. Then $X \in \mathbf{C o r}^{\text {pro }}$. 
Proof. Set $S_{i}=\operatorname{Spec} A_{i}$ and $S=\operatorname{Spec} A=\lim _{i} S_{i}$. Choose an $S$-embedding $X \subset \mathbf{P}_{S}^{n}$. We find an $i_{0}$ and a subscheme $X_{i_{0}} \subset \mathbf{P}_{S_{i_{0}}}^{n}$ such that $X=X_{i_{0}} \times_{S_{i_{0}}} S$. Set $X_{i}:=X_{i_{0}} \times{ }_{S_{i_{0}}} S_{i}$, for $i \geq i_{0}$. Then the transition maps $X_{j} \rightarrow X_{i}, j \geq i \geq i_{0}$, are affine and flat, and hence so is the projection $\tau_{i}: X=\lim _{i} X_{i} \rightarrow X_{i_{0}}$. Since $X$ is regular, there exists an open neighbourhood $U_{i_{0}} \subset X_{i_{0}}$ containing $\tau_{i_{0}}(X)$ which is regular (see [17, Corollaire (6.5.2)]). Since $U_{i_{0}}$ is of finite type over the perfect field $k$, it is even smooth. Set $U_{i}=U_{i_{0}} \times_{S_{i_{0}}} S_{i}$. Then the transition maps $U_{j} \rightarrow U_{i}, j \geq i \geq i_{0}$, are affine and flat, each $U_{i}$ is smooth and we have $X=\lim _{i} U_{i}$; hence $X \in$ Cor $^{\text {pro }}$.

Lemma 2.5. There is (up to isomorphism) a canonical and faithful functor

$$
\text { Cor }^{\text {pro }} \rightarrow \text { ProCor }, \quad \varliminf_{i} X_{i} \mapsto\left(X_{i}\right)
$$

Proof. For any $X \in$ Cor $^{\text {pro }}$ we choose once and for all a projective system $\left(X_{i}\right)_{i \in I}$ as in equation (2.2.1). In particular, $\left(X_{i}\right) \in$ ProCor. Note that if $X=\lim _{j \in J} X_{j}^{\prime}$, then $\left(X_{i}\right) \cong$ $\left(X_{j}^{\prime}\right)$ in ProSm. Take $X=\lim _{i \in I} X_{i}$ and $Y=\lim _{j \in J} Y_{j}$ in Cor ${ }^{\text {pro }}$ and let $V \subset X \times Y$ be a prime correspondence. For any scheme $S$ over $k$, we denote by

$$
\rho_{i}: X \times S \rightarrow X_{i} \times S, \quad \rho_{i^{\prime}, i}: X_{i^{\prime}} \times S \rightarrow X_{i} \times S, \quad i^{\prime} \geq i
$$

and by

$$
\sigma_{j}: Y \times S \rightarrow Y_{j} \times S, \quad \sigma_{j^{\prime}, j}: Y_{j^{\prime}} \times S \rightarrow Y_{j} \times S, \quad j^{\prime} \geq j,
$$

the projection and transition maps of $\left(X_{i} \times S\right)$ and $\left(Y_{j} \times S\right)$, respectively. By assumption, all these maps are affine. For all $j$, the morphism $V \rightarrow X \times Y_{j}$ induced by $\sigma_{j}$ is a morphism of finite-type $X$-schemes. Since $V$ is finite over $X$, its image $\sigma_{j}(V) \subset X \times Y_{j}$ is proper over $X$. Hence $V \rightarrow \sigma_{j}(V)$ is proper and affine, and hence finite. Since $X$ is Noetherian, $\sigma_{j}(V)$ is finite over $X$, and hence we obtain a well-defined correspondence $\sigma_{j *} V \in \operatorname{Cor}\left(X, Y_{j}\right)$ with the property

$$
\sigma_{j *} V=0 \Longleftrightarrow V=0
$$

Furthermore, since $X \times Y_{j}$ is Noetherian, we find an index $i$ (depending on $j$ ) and a correspondence $V_{i, j} \in \mathbf{C o r}\left(X_{i}, Y_{j}\right)$ such that

$$
\sigma_{j *} V=\rho_{i}^{*} V_{i, j}
$$

If we find $i^{\prime}$ and $V_{i^{\prime}, j}^{\prime}$ with $\rho_{i^{\prime}}^{*} V_{i^{\prime}, j}^{\prime}=\sigma_{j *} V$, then clearly $V_{i, j}=V_{i^{\prime}, j}^{\prime}$ in $\lim _{i} \operatorname{Cor}\left(X_{i}, Y_{j}\right)$. Therefore we obtain a well-defined element $V_{j}$

$$
\operatorname{Cor}\left(X_{i}, Y_{j}\right) \rightarrow \underset{i}{\lim } \operatorname{Cor}\left(X_{i}, Y_{j}\right), \quad V_{i, j} \mapsto V_{j} .
$$

By the base-change formula (see equation (2.5.6)), we obtain $\sigma_{j^{\prime}, j *} V_{j^{\prime}}=V_{j}$. We obtain a morphism

$$
\operatorname{Cor}^{\text {pro }}(X, Y) \rightarrow \operatorname{ProCor}\left(\left(X_{i}\right),\left(Y_{j}\right)\right), \quad V \mapsto\left(V_{j}\right)_{j}
$$


It is injective by formula (2.5.1). Finally we have to check that morphism (2.5.2) is compatible with composition. Take $Z=\lim _{l \in L} Z_{l} \in \mathbf{C o r}^{\text {pro. }}$. For any scheme $S$ over $k$, denote by

$$
\tau_{l}: Z \times S \rightarrow Z_{l} \times S
$$

the projection map. Take prime correspondences $V \in \operatorname{Cor}^{\text {pro }}(X, Y)$ and $W \in$ $\operatorname{Cor}^{\operatorname{pro}}(Y, Z)$. For any $l \in L$ we find an index $j(l) \in J$ and a correspondence $W_{j(l), l} \in$ $\operatorname{Cor}\left(Y_{j(l)}, Z_{l}\right)$ such that $\tau_{l *} W=\sigma_{j(l)}^{*} W_{j(l), l}$. For any $j(l)$ we find an index $i(j(l)) \in I$ and a correspondence $V_{i(j(l)), j(l)} \in \operatorname{Cor}\left(X_{i(j(l)), Y_{j(l)}}\right)$ such that $\sigma_{j(l) *} V=\rho_{i(j(l))}^{*} V_{i(j(l)), j(l)}$. Then the compatibility of morphism (2.5.2) will hold if we can show

$$
\tau_{l *}(W \circ V)=\rho_{i(j(l))}^{*}\left(W_{j(l), l} \circ V_{i(j(l)), j(l)}\right), \quad \text { for all } l \in L .
$$

To this end, we recall some well-known formulas. Assume we are given the diagram

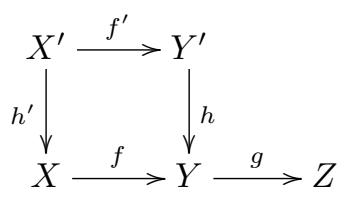

of schemes over $k$ which are in $\mathbf{C o r}^{\text {pro }}$, and assume the square is cartesian and Torindependent. Then for for cycles $\alpha$ on $X, \beta, \beta^{\prime}$ on $Y$, and $\gamma$ on $Z$ the following the following relations hold as soon as both sides of the equation are defined (see [46, V, C]):

$$
\begin{aligned}
f^{*} g^{*} \gamma & =(g \circ f)^{*} \gamma . \\
g_{*} f_{*} \alpha & =(g \circ f)_{*} \alpha . \\
h^{*} f_{*} \alpha & =f_{*}^{\prime} h^{\prime *} \alpha . \\
f^{*}\left(\beta \cdot \beta^{\prime}\right) & =f^{*}(\beta) \cdot f^{*}\left(\beta^{\prime}\right) . \\
f_{*}\left(\alpha \cdot f^{*}(\beta)\right) & =f_{*}(\alpha) \cdot \beta .
\end{aligned}
$$

Using these formulas it is straightforward - but a bit longish - to check that equation (2.5.3) holds. Indeed, since all cycles involved are always finite over some scheme over $k$, it will be clear that the formulas in question are defined; the base-change formula (2.5.6) will be applied only in cases where one of the maps $f$ or $h$ is flat, and hence the Torindependence condition will be automatic. (But note that $h$ might not be flat, so there might appear higher Tors in the computation of $h^{*}$ and $h^{\prime *}$.) This finishes the proof.

2.6. A presheaf with transfers in the sense of Suslin and Voevodsky is a functor $F$ : $\mathbf{C o r}^{\circ} \rightarrow \mathbf{A b}$; they form the category PST. We extend it to a functor $F:$ ProCor $^{\circ} \rightarrow \mathbf{A b}$ by the formula

$$
F\left(\left(X_{i}\right)_{i \in I}\right):=\underset{i}{\lim } F\left(X_{i}\right)
$$


Precomposing $F$ with the functor from Lemma 2.5, we obtain presheaves on Cor ${ }^{\text {pro }}$, which we again denote by $F$,

$$
F:\left(\mathbf{C o r}^{\mathrm{pro}}\right)^{\mathrm{o}} \rightarrow \mathbf{A b}
$$

For $\alpha \in \operatorname{Cor}^{\text {pro }}(X, Y)$, we denote by $\alpha^{*}=F(\alpha): F(Y) \rightarrow F(X)$ the induced map. If $f: X \rightarrow Y$ is a morphism with graph $\Gamma_{f} \subset X \times Y$ between $k$-schemes which are objects in Cor $^{\text {pro }}$, then we set

$$
f^{*}:=\Gamma_{f}^{*}: F(Y) \rightarrow F(X)
$$

if $f$ is a finite morphism and $\Gamma_{f}^{t} \subset Y \times X$ is the transpose of the graph of $f$, we set

$$
f_{*}:=\left(\Gamma_{f}^{t}\right)^{*}: F(X) \rightarrow F(Y) .
$$

\section{Review of reciprocity sheaves}

In this section we collect some definitions, notations and results from [24, 25, 27, 45].

3.1. A modulus pair $\mathcal{X}=\left(\bar{X}, X_{\infty}\right)$ consists of a separated and finite-type $k$-scheme $\bar{X}$ and an effective Cartier divisor $X_{\infty} \geq 0$ such that the open complement $X:=\bar{X} \backslash\left|X_{\infty}\right|$ is smooth. We say $\mathcal{X}$ is a proper modulus pair if $\bar{X}$ is proper over $k$. A basic example is the cube

$$
\bar{\square}:=\left(\mathbf{P}_{k}^{1}, \infty\right)
$$

Let $\mathcal{X}=\left(\bar{X}, X_{\infty}\right)$ and $\mathcal{Y}=\left(\bar{Y}, Y_{\infty}\right)$ be two modulus pairs with corresponding opens $X=\bar{X} \backslash\left|X_{\infty}\right|$ and $Y=\bar{Y} \backslash\left|Y_{\infty}\right|$, respectively. The modulus pair $\mathcal{X} \otimes \mathcal{Y}$ is defined by

$$
\mathcal{X} \otimes \mathcal{Y}:=\left(\bar{X} \times \bar{Y}, X_{\infty} \times \bar{Y}+\bar{X} \times Y_{\infty}\right) .
$$

An admissible prime correspondence from $\mathcal{X}$ to $\mathcal{Y}$ is a prime correspondence $V \in$ $\operatorname{Cor}(X, Y)$ satisfying the condition

$$
X_{\infty \mid \bar{V}^{N}} \geq Y_{\infty \mid \bar{V}^{N}}
$$

where $\bar{V}^{N} \rightarrow \bar{V} \subset \bar{X} \times \bar{Y}$ is the normalisation of the closure of $V$. We denote by $\operatorname{Cor}_{\mathrm{adm}}(\mathcal{X}, \mathcal{Y}) \subset \operatorname{Cor}(X, Y)$ the subgroup generated by admissible correspondences. Assume $\mathcal{X}$ is a proper modulus pair. Recall from [27, Lemma 2.2.2] that the presheaf with transfers $h_{0}(\mathcal{X}) \in \mathbf{P S T}$ is defined by

$$
h_{0}(\mathcal{X})(S)=\operatorname{Coker}\left(\operatorname{Cor}_{\mathrm{adm}}(\bar{\square} \otimes S, \mathcal{X}) \stackrel{i_{0}^{*}-i_{1}^{*}}{\longrightarrow} \operatorname{Cor}(S, X)\right),
$$

where we write $S$ instead of $(S, \emptyset)$ and $i_{\varepsilon}: S \hookrightarrow \mathbf{A}_{S}^{1}$ is the $\varepsilon$-section, $\varepsilon \in\{0,1\}$. We have a natural quotient map $\mathbb{Z}_{\mathrm{tr}}(X) \rightarrow h_{0}(\mathcal{X})$, where $\mathbb{Z}_{\mathrm{tr}}(X)$ is the presheaf with transfers representing $X$ - that is, $\mathbb{Z}_{\operatorname{tr}}(X)(S)=\operatorname{Cor}(S, X)$.

Definition 3.2 ([27, Definition 2.2.4]). Set $F \in \mathbf{P S T}, X \in \mathbf{S m}$ and $a \in F(X)$. We say $a$ has $S C$-modulus (or just modulus) $\mathcal{X}$ if $\mathcal{X}=\left(\bar{X}, X_{\infty}\right)$ is a proper modulus pair with 
$X=\bar{X} \backslash\left|X_{\infty}\right|$ and the Yoneda map $a: \mathbb{Z}_{\mathrm{tr}}(X) \rightarrow F$ factors via

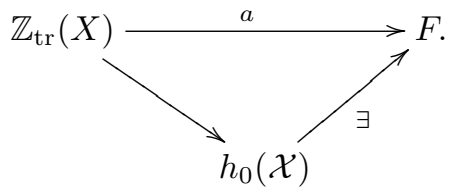

That is, for any $S \in \mathbf{S m}$ and any correspondence $\gamma \in \mathbf{C o r}_{\mathrm{adm}}(\bar{\square} \times S, \mathcal{X}) \subset \mathbf{C o r}\left(\mathbf{A}^{1} \times S, X\right)$ we have $i_{0}^{*} \gamma^{*} a=i_{1}^{*} \gamma^{*} a$.

We say $F$ has $S C$-reciprocity if for all $X \in \mathbf{S m}$ any $a \in F(X)$ has a modulus. We denote by $\mathbf{R S C} \subset$ PST the full subcategory consisting of presheaves with transfers which have SC-reciprocity. Further, we set

\section{$\mathbf{R S C}_{\mathrm{Nis}}=\mathbf{R S C} \cap \mathbf{N S T}$,}

where NST $\subset$ PST is the full subcategory of Nisnevich sheaves with transfers.

3.3. It is shown in [27] that the presheaves in $\mathbf{R S C}$ are in fact induced by presheaves on modulus pairs in the following way: let $\mathcal{X}=\left(\bar{X}, X_{\infty}\right)$ and $\mathcal{Y}=\left(\bar{Y}, Y_{\infty}\right)$ be modulus pairs with corresponding opens $X$ and $Y$, respectively. An admissible correspondence from $\mathcal{X}$ to $\mathcal{Y}$ (see Section 3.1.1) is called left proper if the closure in $\bar{X} \times \bar{Y}$ of all its irreducible components is proper over $\bar{X}$. We denote by $\underline{\operatorname{MCor}}(\mathcal{X}, \mathcal{Y}) \subset \operatorname{Cor}(X, Y)$ the subgroup of all left proper admissible correspondences. This subgroup is stable under composition of correspondences (see [24, Proposition 1.2.3]). Hence we can define the category MCor whose objects are the modulus pairs and with morphisms given by admissible left proper correspondences. We denote by MCor the full subcategory whose objects are the proper

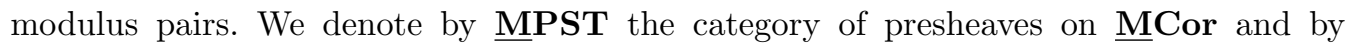
MPST the category of presheaves on MCor. By [24, Proposition 2.2.1, Proposition 2.3.1, Proposition 2.4.1] there are three pairs of adjoint functors $\left(\omega_{!}, \omega^{*}\right),\left(\underline{\omega}_{!}, \underline{\omega}^{*}\right)$ and $\left(\tau_{!}, \tau^{*}\right)$,

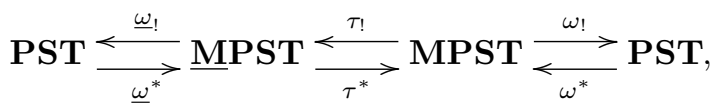

which are given by

$$
\begin{array}{rlrl}
\underline{\omega}^{*} F\left(\bar{X}, X_{\infty}\right) & =F\left(\bar{X} \backslash\left|X_{\infty}\right|\right), & & \underline{\omega}_{!} H(X)=H(X, \emptyset), \\
\omega^{*} F\left(\bar{X}, X_{\infty}\right) & =F\left(\bar{X} \backslash\left|X_{\infty}\right|\right), & \omega_{!} G(X) \cong \lim _{\mathcal{X} \in \overrightarrow{\operatorname{MSm}}(X)} G(\mathcal{X}), \\
\tau^{*} F(\mathcal{X}) & =F(\mathcal{X}), & \tau_{!} G(\mathcal{U}) & \cong \lim _{\mathcal{X} \in \operatorname{Comp}(\mathcal{U})} G(\mathcal{X}),
\end{array}
$$

where $\operatorname{MSm}(X)$ is the subcategory of MCor whose objects are the proper modulus pairs with corresponding opens $X$ and with only those morphism which map to the identity in $\operatorname{Cor}(X, X)$, and $\operatorname{Comp}(\mathcal{U})$ is the category of compactifications of $\mathcal{U}=\left(\bar{U}, U_{\infty}\right)$ - that is, its objects are proper modulus pairs $\mathcal{X}=\left(\bar{X}, \bar{U}_{\infty}+\Sigma\right)$, where $\bar{U}_{\infty}$ and $\Sigma$ are effective Cartier divisors such that $\bar{X} \backslash|\Sigma|=\bar{U}$ and $\bar{U}_{\infty \mid \bar{U}}=U_{\infty}$, and the morphisms are those 
which map to the identity in $\underline{\operatorname{MCor}}(\mathcal{U}, \mathcal{U})$ (see [24, Lemma 2.4.2]). The functors $\omega_{!}, \underline{\omega}_{!}$, $\tau_{!}$are exact and we have $\omega_{!}=\underline{\omega_{!}} \tau_{!}$.

We denote by CI the full subcategory of MPST of cube-invariant objects - that is, those $F \in$ MPST which satisfy the condition that for any proper modulus pair $\mathcal{X}$, the pullback along $\mathcal{X} \otimes \bar{\square} \rightarrow \mathcal{X}$ induces an isomorphism

$$
F(\mathcal{X}) \cong F(\mathcal{X} \otimes \bar{\square})
$$

By [27, Proposition 2.3.7] we have $\omega_{!}(\mathbf{C I})=\mathbf{R S C}$, and there is a fully faithful left exact functor $\omega^{\mathbf{C I}}: \mathbf{R S C} \rightarrow \mathbf{C I}$ given by

$$
\omega^{\mathbf{C I}}(F)\left(\bar{X}, X_{\infty}\right)=\left\{a \in F\left(\bar{X} \backslash X_{\infty}\right) \mid a \text { has modulus }\left(\bar{X}, X_{\infty}\right)\right\} .
$$

We have

$$
\underline{\omega}_{!} \tau ! \omega^{\mathbf{C I}}(F) \cong \omega_{!} \omega^{\mathbf{C I}}(F) \cong F .
$$

3.4. We recall some more definitions and results from [24, 25, 45] related to Nisnevich sheaves.

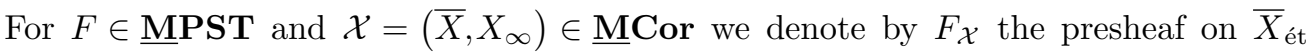
defined by

$$
(U \stackrel{u}{\rightarrow} \bar{X}) \mapsto F_{\mathcal{X}}(U):=F\left(U, u^{*} X_{\infty}\right) .
$$

We denote by MNST the full subcategory of MPST consisting of those $F$ such that

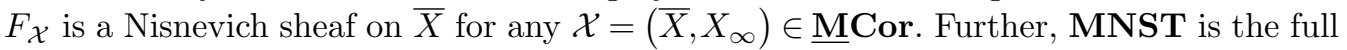

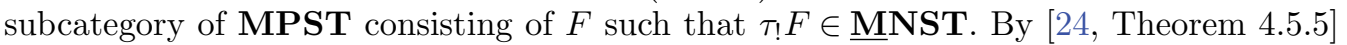
and [25, Theorem 4.2.4], there are exact sheafification functors (that is, left adjoints to the natural inclusions)

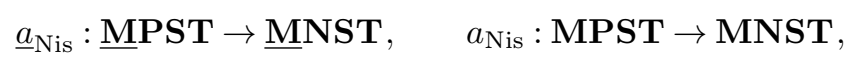

such that

(1) $\left(\underline{a}_{\mathrm{Nis}} F\right)(\mathcal{X})=\underline{\lim }_{\rightarrow: Y} \stackrel{\sim}{\rightarrow} \bar{X} F_{\mathcal{X}, \mathrm{Nis}}\left(Y, f^{*} X_{\infty}\right)$, where $\mathcal{X}=\left(\bar{X}, X_{\infty}\right) \in \underline{\text { MCor }}, F \mathcal{X}$, Nis denotes the Nisnevich sheafification of the presheaf $F_{\mathcal{X}}$ on $\bar{X}_{\text {ét }}$, and the limit is over all proper birational morphisms $f: Y \rightarrow \bar{X}$ which restrict to an isomorphism $Y \backslash\left|f^{*} X_{\infty}\right| \stackrel{\simeq}{\rightarrow} \bar{X} \backslash\left|X_{\infty}\right|$

(2) $\tau_{!}$restricts to an exact functor $\tau_{!}:$MNST $\rightarrow \underline{\text { MNST } \text { and satisfies }}$

$$
\underline{a}_{\mathrm{Nis}} \tau_{!} F=\tau_{!} a_{\mathrm{Nis}} F \quad \text { for all } F \in \text { MPST } .
$$

It follows that $a_{\mathrm{Nis}}=\tau^{*} \underline{a}_{\mathrm{Nis}} \tau_{\text {! }}$ and

$$
\underline{a}_{\mathrm{Nis}\lceil\underline{\mathrm{MNST}}}=\mathrm{id}_{\underline{\mathbf{M N S T}}}, \quad a_{\mathrm{Nis} \uparrow \mathrm{MNST}}=\mathrm{id}_{\mathrm{MNST}} .
$$

By [25, Proposition 6.2.1],

$$
\underline{a}_{\mathrm{Nis}} \underline{\omega}^{*}=\underline{\omega}^{*} a_{\mathrm{Nis}}^{V}, \quad a_{\mathrm{Nis}} \omega^{*}=\omega^{*} a_{\mathrm{Nis}}^{V},
$$


where $a_{\mathrm{Nis}}^{V}: \mathbf{P S T} \rightarrow \mathbf{N S T}$ is Voevodsky's Nisnevich sheafification functor (see [49, Lemma 3.1.6]), and we obtain induced functors

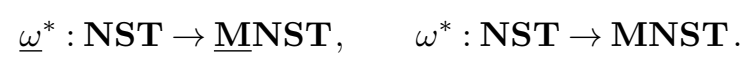

Lemma 3.5. For $F \in \mathbf{R S C}_{\text {Nis }}$, we have $\omega^{\mathbf{C I}} F \subset a_{\mathrm{Nis}} \omega^{\mathbf{C I}} F \subset \omega^{*} F$ in MPST (see Definition 3.2 and equation (3.3.4) for notation). Here the first inclusion is given by the unit of adjunction.

Proof. By definition, $\omega^{\mathbf{C I}} F \subset \omega^{*} F$. We obtain the commutative diagram

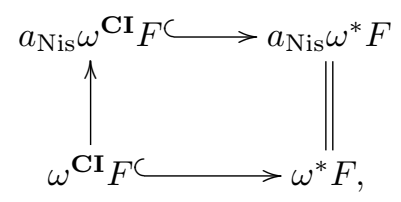

in which the vertical maps are induced by adjunction. The vertical map on the right is an isomorphism, since $\omega^{*} F \in \mathbf{M N S T}$, and the top horizontal map is an inclusion, since $a_{\mathrm{Nis}}$ is exact. This gives the statement.

Remark 3.6. It follows from Corollary 4.16 that the first inclusion in Lemma 3.5 is actually an equality.

3.7. We define the category $\underline{\mathbf{M C o r}}^{\text {pro }}$ as follows: the objects are pairs $\mathcal{X}=\left(\bar{X}, X_{\infty}\right)$, where

(1) $\bar{X}$ is a separated Noetherian scheme over $k$ of the form $\bar{X}=\lim _{i \in I} \bar{X}_{i}$, with $\left(\bar{X}_{i}\right)_{i \in I}$ a projective system of separated finite-type $k$-schemes indexed by a partially ordered set with affine transition maps $\tau_{i, j}: \bar{X}_{i} \rightarrow \bar{X}_{j}, i \geq j$;

(2) $X_{\infty}=\lim _{i \in I} X_{i, \infty}$, with $X_{i, \infty}$ an effective Cartier divisor on $\bar{X}_{i}$, such that $\bar{X}_{i} \backslash$ $\left|X_{i, \infty}\right|$ is smooth for all $i$ and $\tau_{i, j}^{*} X_{j, \infty}=X_{i, \infty}, i \geq j$

(3) $\bar{X} \backslash\left|X_{\infty}\right|$ is regular.

The morphisms are given by the admissible left proper correspondences, which are verbatim defined as in Section 3.3. That the composition of correspondences in Cor $^{\text {pro }}$ induces a well-defined composition in $\underline{\mathbf{M C o r}}^{\text {pro }}$ is shown in the same way as in [24, Proposition 1.2.3].

Lemma 3.8. There is (up to isomorphism) a canonical and faithful functor

$$
\underline{\text { MCor }}{ }^{\text {pro }} \rightarrow \text { ProMCor }, \quad \underset{i}{\lim }\left(\bar{X}_{i}, X_{i, \infty}\right) \mapsto\left(\bar{X}_{i}, X_{i, \infty}\right)_{i} \text {. }
$$

Proof. Let $\mathcal{X}=\left(\bar{X}, X_{\infty}\right), \mathcal{Y}=\left(\bar{Y}, Y_{\infty}\right) \in$ ProMCor. We write $\mathcal{X}=\lim _{i \in I} \mathcal{X}_{i}$ with $\mathcal{X}_{i}=$ $\left(\bar{X}_{i}, X_{i, \infty}\right)$, and similarly $\mathcal{Y}=\lim _{j \in J} \mathcal{Y}_{j}$. Set $X=\bar{X} \backslash\left|X_{\infty}\right|$, and so on. We have to show that the injection $(2.5 .2)$ restricts to

$$
\underline{\operatorname{MCor}}^{\mathrm{pro}}(\mathcal{X}, \mathcal{Y}) \rightarrow \operatorname{Pro\underline {MCor}}\left(\left(\mathcal{X}_{i}\right),\left(\mathcal{Y}_{j}\right)\right) .
$$


To this end, let $V \in \underline{\mathbf{M}} \operatorname{Cor}^{\text {pro }}(\mathcal{X}, \mathcal{Y})$ be a left proper admissible correspondence. For $j \in J$, denote by $\sigma_{j}(V)$ the image of $V$ under the projection $X \times Y \rightarrow X \times Y_{j}$. Then $\sigma_{j}(V)$ is a finite prime correspondence, as was observed in the proof of Lemma 2.5. Let $\bar{V} \subset \bar{X} \times \bar{Y}$ be the closure of $V$. By assumption, $\bar{V}$ is proper over $X$. Since $\bar{X} \times Y_{j}$ is separated and of finite type over $\bar{X}$, the image of $\bar{V}$ in $\bar{X} \times Y_{j}$ is closed and proper over $X$; hence it is equal to the closure $\overline{\sigma_{j}(V)}$ of $\sigma_{j}(V)$. Now [24, Lemma 1.2.1] yields

$$
X_{\infty}{\overline{\sigma_{j}(V)}}^{N} \geq Y_{j, \infty}{\overline{\sigma_{j}(V)}}^{N},
$$

with the notation from formula (3.1.2). As in the proof of Lemma 2.5, we find an index $i_{0} \in I$ and a finite correspondence $V_{i_{0}, j} \subset X_{i_{0}} \times Y_{j}$ which pulls back to $\sigma_{j}(V)$. We can also assume (after possibly enlarging $i_{0}$ ) that the closure $\bar{V}_{i_{0}, j} \subset \bar{X}_{i_{0}} \times \bar{Y}_{j}$ of $V_{i_{0}, j}$ pulls back to $\overline{\sigma_{j}(V)}$. We obtain the cartesian diagram

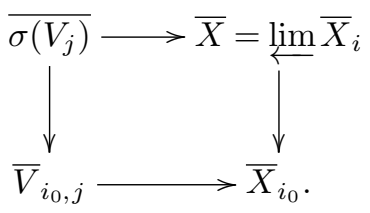

Since the upper horizontal arrow is proper, the lower horizontal arrow becomes proper after possibly enlarging $i_{0}$ (see $[18$, Théorème (8.10.5), (xii)]). Hence, by our construction and formula (3.8.2), the scheme $V_{i_{0}, j}=\bar{V}_{i_{0}, j} \cap\left(X_{i_{0}} \times Y_{j}\right)$ is a left proper admissible correspondence from $\mathcal{X}_{i_{0}}$ to $\mathcal{Y}_{j}$ and gives a well-defined element

$$
V_{j} \in \underset{i \in I}{\lim } \operatorname{ProM} \operatorname{Cor}\left(\mathcal{X}_{i}, \mathcal{Y}_{j}\right)
$$

This shows that morphism (2.5.2) restricts to formula (3.8.1).

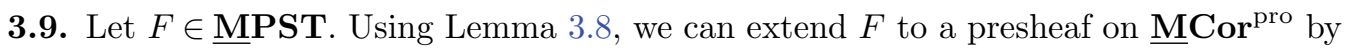
the formula

$$
F(\mathcal{X})=\underset{i}{\lim } F\left(\mathcal{X}_{i}\right), \quad \mathcal{X}={\underset{\leftarrow}{i}}_{\lim _{i}} \mathcal{X}_{i} \underline{\mathbf{M C o r}}^{\text {pro }}
$$

\section{Conductors for presheaves with transfers}

\section{Definition 4.1.}

(1) We say that $L$ is a henselian discrete valuation field of geometric type (over $k$ ) (or that $L$ is a henselian $d v f$ ) if it is a discrete valuation field and its ring of integers is equal to the henselisation of the local ring of a smooth $k$-scheme $U$ in a 1codimensional point $x \in U^{(1)}$ - that is, $\mathcal{O}_{L}=\mathcal{O}_{U, x}^{h}$. For $n \in \mathbb{N}_{\geq 1} \cup\{\infty\}$, we set

$$
\Phi=\{L \text { henselian dvf }\}, \quad \Phi_{\leq n}=\{L \in \Phi \mid \operatorname{trdeg}(L / k) \leq n\} .
$$

Note that in positive characteristic, $\Phi_{\leq 1}$ consists precisely of the henselian dvfs with perfect residue field. 
(2) Let $X$ be a smooth $k$-scheme. A henselian $d v f$ point of $X$ is a $k$-morphism $\operatorname{Spec} L \rightarrow$ $X$, with $L \in \Phi$.

(3) Let $\mathcal{X}=\left(\bar{X}, X_{\infty}\right)$ be a modulus pair with $X=\bar{X} \backslash\left|X_{\infty}\right|$. A henselian dvf point of $\mathcal{X}$ is a henselian dvf point $\rho: \operatorname{Spec} L \rightarrow X$ extending to $\operatorname{Spec} \mathcal{O}_{L} \rightarrow \bar{X}$. Note, if it exits, such an extension is unique, and if $\mathcal{X}$ is proper, then there always exists an extension. We will denote this extension also by $\rho$. We will also write $\rho: \operatorname{Spec} L \rightarrow \mathcal{X}$ for the henselian dvf point of $\mathcal{X}$ defined by $\rho$.

\section{Notation 4.2.}

(1) Set $F \in \mathbf{P S T}$ and $X \in \mathbf{S m}$. A henselian dvf point $\rho: \eta=\operatorname{Spec} L \rightarrow X$ is a morphism in Cor $^{\text {pro }}$ (see Defintion 2.2). Hence we get a morphism (see Section 2.6)

$$
\rho^{*}: F(X) \rightarrow F(\eta)=: F(L), \quad a \mapsto \rho^{*} a .
$$

Also, $\eta=\operatorname{Spec} L \rightarrow \operatorname{Spec} \mathcal{O}_{L}=\bar{\eta}$ is in $\operatorname{Cor}^{\text {pro }}$, and we get an induced map $F\left(\mathcal{O}_{L}\right):=$ $F(\bar{\eta}) \rightarrow F(L)$.

(2) Let $\mathcal{X}=\left(\bar{X}, X_{\infty}\right)$ be a modulus pair with $X=\bar{X} \backslash\left|X_{\infty}\right|$ and $\rho: \operatorname{Spec} L \rightarrow \mathcal{X}$ a henselian dvf point. Then we denote by

$$
v_{L}\left(X_{\infty}\right)=v\left(\rho^{*} X_{\infty}\right) \in \mathbb{N}_{0}
$$

the multiplicity of $X_{\infty}$ pulled back along $\bar{\rho}$.

Definition 4.3. Set $F \in \mathbf{P S T}$ and $n \in[1, \infty]$. A conductor of level $n$ for $F$ is a collection of set maps

$$
c=\left\{c_{L}: F(L) \rightarrow \mathbb{N}_{0} \mid L \in \Phi_{\leq n}\right\}
$$

satisfying the following properties for all $L \in \Phi_{\leq n}$ and all $X \in \mathbf{S m}$ :

(c1) $c_{L}(a)=0 \Rightarrow a \in \operatorname{Im}\left(F\left(\mathcal{O}_{L}\right) \rightarrow F(L)\right)$.

(c2) $c_{L}(a+b) \leq \max \left\{c_{L}(a), c_{L}(b)\right\}$.

(c3) $c_{L}\left(f_{*} a\right) \leq\left[\frac{c_{L^{\prime}}(a)}{e\left(L^{\prime} / L\right)}\right]$ for any finite morphism $f: \operatorname{Spec} L^{\prime} \rightarrow \operatorname{Spec} L$ and any $a \in F\left(L^{\prime}\right)$. Here $e\left(L^{\prime} / L\right)$ denotes the ramification index of $L^{\prime} / L$ and $\lceil-\rceil$ is the roundin up.

(c4) Assume $a \in F\left(\mathbf{A}_{X}^{1}\right)$ satisfies $c_{k(x)(t)_{\infty}}\left(\rho_{x}^{*} a\right) \leq 1$ for all $x \in X$ with $\operatorname{trdeg}(k(x) / k) \leq$ $n-1$, where $k(x)(t)_{\infty}:=\operatorname{Frac}\left(\mathcal{O}_{\mathbf{P}_{x}^{1}, \infty}^{h}\right)$ and $\rho_{x}: \operatorname{Spec} k(x)(t)_{\infty} \rightarrow \mathbf{A}_{X}^{1}$ is the natural map. Then $a \in \pi^{*} F(X)$, with $\pi: \mathbf{A}_{X}^{1} \rightarrow X$ the projection.

(c5) For any $a \in F(X)$ there exists a proper modulus pair $\mathcal{X}=\left(\bar{X}, X_{\infty}\right)$ with $X=$ $\bar{X} \backslash\left|X_{\infty}\right|$, such that for all $\rho: \operatorname{Spec} L \rightarrow \mathcal{X}$ we have

$$
c_{L}\left(\rho^{*} a\right) \leq v_{L}\left(X_{\infty}\right) .
$$

A conductor of level $\infty$ will be simply called a conductor.

\section{Remarks 4.4.}

(1) If $F$ is homotopy invariant, then setting $c_{L}(a)=0$ if $a \in \operatorname{Im}\left(F\left(\mathcal{O}_{L}\right) \rightarrow F(L)\right)$ and $c_{L}(a)=1$ otherwise defines a conductor (of any level). 
(2) If $c=\left\{c_{L}\right\}$ is a conductor for $F$, then for any $L$ we have

$$
a \in \operatorname{Im}\left(F\left(\mathcal{O}_{L}\right) \rightarrow F(L)\right) \Longleftrightarrow c_{L}(a)=0 .
$$

Indeed, if $a \in \operatorname{Im}\left(F\left(\mathcal{O}_{L}\right) \rightarrow F(L)\right)$, then we find a smooth $k$-scheme $U$, a 1 codimensional point $x \in U^{(1)}$, a $k$-morphism $\operatorname{Spec} \mathcal{O}_{L} \rightarrow \operatorname{Spec} \mathcal{O}_{U, x} \rightarrow U$ and an element $\tilde{a} \in F(U)$ such that $\rho^{*} \tilde{a}=a \in F(L)$, where $\rho: \operatorname{Spec} L \rightarrow \operatorname{Spec} \mathcal{O}_{L} \rightarrow U$. The vanishing of $c_{L}(a)$ hence follows directly from axiom (c5).

(3) Let $c=\left\{c_{L}\right\}$ be a conductor. Then $c^{\leq n}:=\left\{c_{L} \mid \operatorname{trdeg}(L / k) \leq n\right\}$ is a conductor if and only if $c^{\leq n}$ satisfies axiom (c4).

Definition 4.5. Set $F \in \mathbf{P S T}$ and let $c=\left\{c_{L}\right\}$ be a conductor of level $n$ for $F$. Let $\mathcal{X}=\left(\bar{X}, X_{\infty}\right)$ be a modulus pair with $X=\bar{X} \backslash\left|X_{\infty}\right|$. For $a \in F(X)$, we write

$$
c_{\bar{X}}(a) \leq X_{\infty}
$$

to mean $c_{L}\left(\rho^{*} a\right) \leq v_{L}\left(X_{\infty}\right)$ for all henselian dvf points $\rho$ : $\operatorname{Spec} L \rightarrow \mathcal{X}$ with $\operatorname{trdeg}(L / k) \leq n$ (see Definition 4.1).

Lemma 4.6. Let $c$ be a conductor of some level for $F \in \mathbf{P S T}, X \in \mathbf{S m}$ and $a \in F(X)$. Let $\mathcal{X}=\left(\bar{X}, X_{\infty}\right)$ be any proper modulus pair with $X=\bar{X} \backslash X_{\infty}$. Then there exists a natural number $n \geq 1$ such that $c_{\bar{X}}(a) \leq n \cdot X_{\infty}$.

Proof. By Definition 4.3, axiom (c5), there exists a proper modulus pair $\mathcal{X}_{1}=\left(\bar{X}_{1}, X_{1, \infty}\right)$ with corresponding open $X$ and such that $c_{L}\left(\rho^{*} a\right) \leq v_{L}\left(X_{1, \infty}\right)$ for all $\rho$. We find a proper normal $k$-scheme $\bar{X}_{2}$ with $k$-morphisms $f: \overline{X_{2}} \rightarrow \bar{X}, f_{1}: \overline{X_{2}} \rightarrow \overline{X_{1}}$ such that $\overline{X_{2}} \backslash\left|f^{*} X_{\infty}\right|=$ $X=\bar{X}_{2} \backslash\left|f_{1}^{*} X_{1, \infty}\right|$. Take $n \geq 1$ with $f_{1}^{*} X_{1, \infty} \leq n \cdot f^{*} X_{\infty}$. Then for $\rho: \operatorname{Spec} L \rightarrow X$,

$$
c_{L}\left(\rho^{*} a\right) \leq v_{L}\left(X_{1, \infty}\right)=v_{L}\left(f_{1}^{*} X_{1, \infty}\right) \leq v_{L}\left(n \cdot f^{*} X_{\infty}\right)=v_{L}\left(n \cdot X_{\infty}\right) .
$$

Hence the statement.

Proposition 4.7. Set $F \in \mathbf{P S T}$ and let $c$ be a conductor of level $n$ for $F$. Then

$$
\text { MCor } \ni \mathcal{X}=\left(\bar{X}, X_{\infty}\right) \mapsto F_{c}(\mathcal{X}):=\left\{a \in F\left(\bar{X} \backslash\left|X_{\infty}\right|\right) \mid c_{\bar{X}}(a) \leq X_{\infty}\right\}
$$

defines an object in MPST. Furthermore (see Section 3.3 for notations), the following are true:

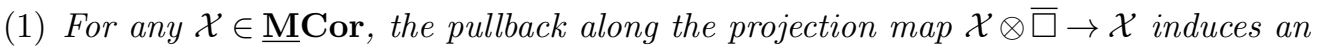
isomorphism $F_{c}(\mathcal{X}) \cong F_{c}(\mathcal{X} \otimes \bar{\square})$. In particular, $\tau^{*} F_{c} \in \mathbf{C I}$.

(2) $\omega_{!} \tau^{*} F_{c} \cong F$.

(3) $F \in \mathbf{N S T} \Rightarrow F_{c} \in \underline{\mathbf{M N S T}}$.

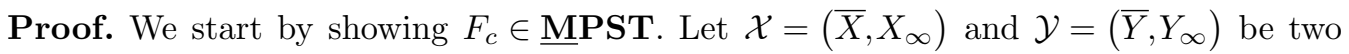
modulus pairs with corresponding opens $X$ and $Y$, respectively. We have to show that a left proper admissible prime correspondence $V \in \underline{\operatorname{M}} \operatorname{Cor}(\mathcal{X}, \mathcal{Y}) \subset \operatorname{Cor}(X, Y)$ sends the subgroup $F_{c}(\mathcal{Y}) \subset F(Y)$ to $F_{c}(\mathcal{X}) \subset F(X)$. Take $a \in F_{c}(\mathcal{Y})$ and a henselian dvf point $\rho: \eta=\operatorname{Spec} L \rightarrow \mathcal{X}$ with $\operatorname{trdeg}(L / k) \leq n$. We have to show

$$
c_{L}\left(\rho^{*} V^{*} a\right) \leq v\left(\bar{\rho}^{*} X_{\infty}\right)
$$


Since $V \rightarrow X$ is finite, $\left(\eta \times_{X} V\right)_{\text {red }}$ is a disjoint union of points $\eta_{i}=\operatorname{Spec} L_{i}$, with $L_{i} \in \Phi_{\leq n}$. Thus

$$
V \circ \rho=\sum_{i} m_{i} \cdot \eta_{i} \in \mathbf{C o r}(\eta, Y)
$$

with some multiplicities $m_{i} \in \mathbb{N}$. For each $i$ we get a commutative diagram

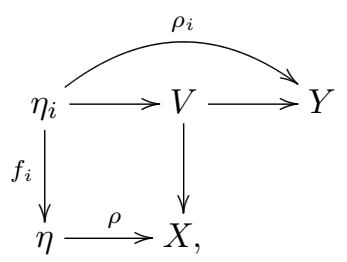

where $\rho_{i}$ is a henselian dvf point of $Y$ and $f_{i}$ is finite. We have $\eta_{i}=\Gamma_{\rho_{i}} \circ \Gamma_{f_{i}}^{t}$ in $\operatorname{Cor}(\eta, Y)$ (see Section 2.6 for the notation). Thus

$$
\rho^{*} V^{*}=\sum_{i} m_{i} \cdot f_{i *} \rho_{i}^{*}: F(Y) \rightarrow F(\eta)
$$

Since the closure $\bar{V}$ of $V$ in $\bar{X} \times \bar{Y}$ is proper over $\bar{X}$ and $\rho$ extends to $\bar{\rho}$, we see that $\rho_{i}$ extends to $\bar{\rho}_{i}$ as in the diagram

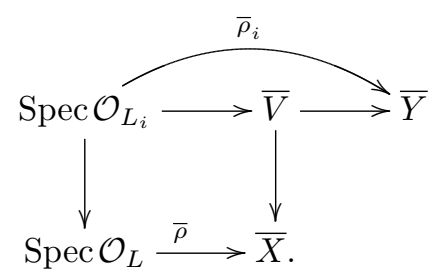

Since $V$ satisfies the modulus condition (3.1.2), we get

$$
v_{L_{i}}\left(X_{\infty}\right) \geq v_{L_{i}}\left(Y_{\infty}\right)
$$

Indeed, let $B$ be the local ring of $\bar{V}$ at the image of the closed point of $\mathcal{O}_{L_{i}}, x$ and $y \in B$ be the local equations for $X_{\infty} / \bar{V}$ and $Y_{\infty} / \bar{V}$, respectively, and $\bar{x}$ and $\bar{y}$ be their images in $\mathcal{O}_{L_{i}} \backslash\{0\}$. Then condition (3.1.2) says that $x / y \in \operatorname{Frac}(B)$ is a root of a monic polynomial $P(T) \in B[T]$. It follows that $\bar{x} / \bar{y} \in L_{i}$ is a root of the image of $P(T)$ under $B[T] \rightarrow \mathcal{O}_{L_{i}}[T]$ - that is, $\bar{x} / \bar{y}$ is integral over $\mathcal{O}_{L_{i}}$, which means $v_{L_{i}}(\bar{x}) \geq v_{L_{i}}(\bar{y})$. 
Let $j$ be an index with $c_{L}\left(f_{j *} \rho_{j}^{*} a\right)=\max _{i}\left\{c_{L}\left(f_{i *} \rho_{i}^{*} a\right)\right\}$. We obtain

$$
\begin{aligned}
c_{L}\left(\rho^{*} V^{*} a\right) & =c_{L}\left(\sum_{i} m_{i} \cdot f_{i *} \rho_{i}^{*} a\right) & & \text { by equation }(4.7 .2), \\
& \leq c_{L}\left(f_{j *} \rho_{j}^{*} a\right) & & \text { by Definition 4.3, axiom (c2), } \\
& \leq\left\lceil\frac{c_{L_{j}}\left(\rho_{j}^{*} a\right)}{e\left(L_{j} / L\right)}\right] & & \text { by Definition 4.3, axiom (c3), } \\
& \leq\left\lceil\frac{v_{L_{j}}\left(Y_{\infty}\right)}{e\left(L_{j} / L\right)}\right\rceil, & & a \in F_{c}(\mathcal{Y}), \\
& \leq\left\lceil\frac{v_{L_{j}}\left(X_{\infty}\right)}{e\left(L_{j} / L\right)}\right\rceil & & \text { by formula }(4.7 .3) \\
& =v_{L}\left(X_{\infty}\right), & &
\end{aligned}
$$

where the last equality follows from $v_{L_{j}}\left(X_{\infty}\right)=e\left(L_{j} / L\right) v_{L}\left(X_{\infty}\right)$. This proves formula (4.7.1) and hence that $F_{c}$ is in MPST.

Next we prove statement $(1)$ in the proposition. Let $\mathcal{X}=\left(\bar{X}, X_{\infty}\right)$ be a modulus pair with $X=\bar{X} \backslash\left|X_{\infty}\right|$. Denote by $\pi: X \times \mathbf{A}_{k}^{1} \rightarrow X$ the projection and by $i_{0}: X \hookrightarrow X \times \mathbf{A}_{k}^{1}$ the zero section. These define morphisms $\pi \in \underline{\operatorname{MCor}}(\mathcal{X} \otimes \bar{\square}, \mathcal{X})$ and $i_{0} \in \underline{\operatorname{MCor}}(\mathcal{X}, \mathcal{X} \otimes \bar{\square})$. We have to show that $\pi^{*}: F_{c}(\mathcal{X}) \rightarrow F_{c}(\mathcal{X} \otimes \bar{\square})$ is an isomorphism. Since $i_{0}^{*} \pi^{*}=\operatorname{id}_{F_{c}(\mathcal{X})}$, it suffices to show that $\pi^{*}$ is surjective. Take $a \in F_{c}(\mathcal{X} \otimes \bar{\square})$. For any henselian dvf point $\rho: \operatorname{Spec} L \rightarrow\left(\mathbf{P}_{X}^{1},\{\infty\}_{X}\right)$ with $\operatorname{trdeg}(L / k) \leq n$, we have

$$
c_{L}\left(\rho^{*} a\right) \leq v_{L}\left(X_{\infty} \times \mathbf{P}^{1}+\bar{X} \times\{\infty\}\right)=v_{L}(X \times\{\infty\}) .
$$

Hence by Definition 4.3, axiom (c4), there exists an element $b \in F(X)$ with $\pi^{*}(b)=a$. We have to check that $b \in F_{c}(\mathcal{X})$. Take $\rho: \operatorname{Spec} L \rightarrow \mathcal{X}$, a henselian $\operatorname{dvf}$ point with $\operatorname{trdeg}(L / k) \leq$ $n$. Then $i_{0} \circ \rho: \operatorname{Spec} L \rightarrow \mathcal{X} \otimes \bar{\square}$ is a henselian dvf point, and thus

$$
\begin{aligned}
c_{L}\left(\rho^{*} b\right) & =c_{L}\left(\rho^{*} i_{0}^{*} \pi^{*} b\right)=c_{L}\left(\left(i_{0} \circ \rho\right)^{*} a\right) \\
& \leq v_{L}\left(X_{\infty} \times \mathbf{P}^{1}+\bar{X} \times\{\infty\}\right)=v_{L}\left(X_{\infty}\right) .
\end{aligned}
$$

Hence $b \in F_{c}(\mathcal{X})$. Statement (2) follows directly from equation (3.3.2) and Definition 4.3, axiom (c5). Finally we address statement $(3)$. For $\mathcal{X}=\left(\bar{X}, X_{\infty}\right)$, the presheaf $F_{c, \mathcal{X}}$ on $\bar{X}_{\text {ét }}$ (see formula (3.4.1)) is given by

$$
(U \stackrel{u}{\rightarrow} \bar{X}) \mapsto\left\{a \in F\left(U \backslash\left|u^{*} X_{\infty}\right|\right) \mid c_{U}(a) \leq u^{*} X_{\infty}\right\}
$$

We have to show that this is a Nisnevich sheaf. Since $F$ is a Nisnevich sheaf, it suffices to show the following: let $u: U \rightarrow \bar{X}$ be an étale map, set $a \in F\left(U \backslash\left|u^{*} X_{\infty}\right|\right)$ and assume there is a Nisnevich cover $\sqcup_{i} U_{i} \stackrel{\sqcup u_{i}}{\longrightarrow} U$ so that $c_{U_{i}}\left(u_{i}^{*} a\right) \leq u_{i}^{*} u^{*} X_{\infty}$ for all $i$. Then we have to show $c_{U}(a) \leq u^{*} X_{\infty}$. To this end, observe that if $\rho$ : $\operatorname{Spec} L \rightarrow\left(U, u^{*} X_{\infty}\right)$ is a henselian $\operatorname{dvf}$ point with $\operatorname{trdeg}(L / k) \leq n$ and $x \in U$ is the image point of the closed point of Spec $\mathcal{O}_{L}$, then by the functoriality of henselisation, $\bar{\rho}$ factors via $\operatorname{Spec} \mathcal{O}_{L} \rightarrow \operatorname{Spec} \mathcal{O}_{U, x}^{h} \rightarrow U$. Hence 
there is an $i$ such that $\bar{\rho}$ factors via $\operatorname{Spec} \mathcal{O}_{L} \rightarrow U_{i} \stackrel{u_{i}}{\longrightarrow} U$. Thus $c_{L}\left(\rho^{*} a\right) \leq v_{L}\left(u_{i}^{*} v^{*} X_{\infty}\right)=$ $v_{L}\left(v^{*} X_{\infty}\right)$. This completes the proof.

4.8. Set $F \in \mathbf{P S T}$ and let $c$ be a conductor of some level for $F$. Let $F_{c} \in \underline{\text { MPST be as }}$ in Proposition 4.7. We set (see Section 3.3 for notation)

$$
\tilde{F}_{c}:=\tau_{!} \tau^{*} F_{c} \in \underline{\text { MPST }} .
$$

By adjunction we have a natural map

$$
\tilde{F}_{c} \rightarrow F_{c}
$$

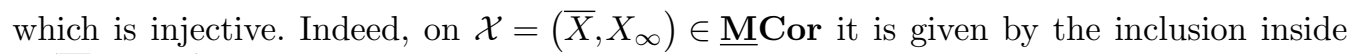
$F\left(\bar{X} \backslash\left|X_{\infty}\right|\right)$,

$$
\tilde{F}_{c}(\mathcal{X})=\underset{\mathcal{Y} \in \operatorname{Comp}(\mathcal{X})}{\lim _{\longrightarrow}} F_{c}(\mathcal{Y}) \rightarrow F_{c}(\mathcal{X}) .
$$

By Proposition 4.7 and [25, Lemma 4.2.5] (or a similar argument as in the proof of Proposition 4.7(3)), we have

$$
F \in \mathbf{N S T} \Rightarrow \tilde{F}_{c} \in \underline{\mathbf{M N S T}} .
$$

4.9. Set $F \in \mathbf{R S C}$. Denote by $\mathbf{C I}(F)$ the partially ordered set consisting of those subobjects $G \subset \omega^{\mathbf{C I}} F$ in MPST such that the induced map $\omega_{!} G \rightarrow \omega_{!} \omega^{\mathbf{C I}} F=F$ is an isomorphism and the partial order is given by the inclusion $G_{1} \subset G_{2}$ We set

$$
\mathbf{C I}(F)_{\mathrm{Nis}}:=\mathbf{C I}(F) \cap \mathbf{M N S T} .
$$

Lemma 4.10. Set $F \in \mathbf{R S C}$ and $G \in \mathbf{C I}(F)$. Then $G_{1}=\tau_{!} G \in \underline{\text { MPST }}$ has the following properties:

(1) the unit $G_{1} \hookrightarrow \underline{\omega}^{*} \underline{\omega}_{!} G_{1}$ of the adjunction $\left(\underline{\omega}_{!}, \underline{\omega}^{*}\right)$ is injective;

(2) the counit $\tau_{!} \tau^{*} G_{1} \stackrel{\simeq}{\rightarrow} G_{1}$ of the adjunction $\left(\tau_{!}, \tau^{*}\right)$ is an isomorphism;

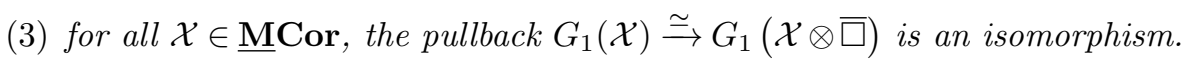

Proof. Note that (2) follows directly from $\tau^{*} \tau_{!}=$id. We show (1) and (3). The inclusion $G \hookrightarrow \omega^{\mathbf{C I}} F$ yields a commutative diagram

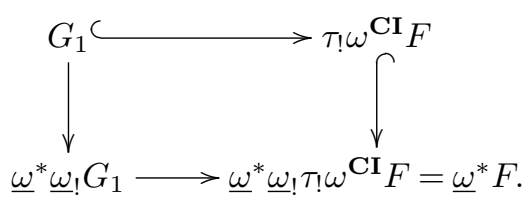

Here the top horizontal row is injective by the exactness of $\tau_{!}$, the vertical maps are induced by adjunction and the vertical map on the right is injective by equation (3.3.4). It follows that the vertical map on the left is injective; furthermore, the injectivity of the top horizontal map and [45, Lemma 1.15 , Lemma 1.16] imply that $G_{1}$ is $\bar{\square}$-invariant.

Remark 4.11. Lemma 4.10 says that $\tau_{!} \mathbf{C I}(F) \subset \tau{ }^{\tau} \mathbf{I}^{s p}$, in the notation of [45]. 
Lemma 4.12. Set $F \in \mathbf{P S T}$ and let $c$ be a conductor of some level for $F$. Then $\tau^{*} \tilde{F}_{c}=$ $\tau^{*} F_{c} \in \mathbf{C I}(F)$ (see Section 4.8 for notation). If $F \in \mathbf{N S T}$, then $\tau^{*} F_{c} \in \mathbf{C I}(F)_{\mathrm{Nis}}$.

Proof. By Proposition 4.7(2), it suffices to show that there is an inclusion $\tau^{*} F_{c} \hookrightarrow \omega^{\mathbf{C I}} F$ inside $\omega^{*} F$. For $\mathcal{X}$ a proper modulus pair, set $\mathbb{Z}_{\mathrm{tr}}(\mathcal{X}):=\operatorname{MCor}(-, \mathcal{X})$ and

$$
h_{0}^{\bar{\square}}(\mathcal{X})=\operatorname{Coker}\left(\mathbb{Z}_{\mathrm{tr}}(\mathcal{X})(\bar{\square} \otimes-) \stackrel{i_{0}^{*}-i_{1}^{*}}{\longrightarrow} \mathbb{Z}_{\mathrm{tr}}(\mathcal{X})\right) .
$$

By [24, Lemma 1.1.3] and [27, Lemma 2.2.2], we have (see Sections 3.1 and 3.3 for notation)

$$
\omega ! \mathbb{Z}_{\mathrm{tr}}(\mathcal{X})=\mathbb{Z}_{\mathrm{tr}}(X), \quad \omega_{!} h_{0}^{\bar{\square}}(\mathcal{X})=h_{0}(\mathcal{X}),
$$

where $X=\bar{X} \backslash\left|X_{\infty}\right|$. Take $a \in F_{c}(\mathcal{X}) \subset F(X)$. Since $F_{c}$ is cube invariant, by Proposition 4.7 , the Yoneda map $a: \mathbb{Z}_{\mathrm{tr}}(\mathcal{X}) \rightarrow \tau^{*} F_{c}$ factors via the quotient map $\mathbb{Z}_{\mathrm{tr}}(\mathcal{X}) \rightarrow h_{0}^{\bar{\square}}(\mathcal{X})$. Applying $\omega_{!}=\underline{\omega}_{!} \tau_{\text {! }}$ we see that the Yoneda map $a: \mathbb{Z}_{\mathrm{tr}}(X) \rightarrow F$ in PST defined by $a \in F(X)$ factors via $\mathbb{Z}_{\mathrm{tr}}(X) \rightarrow h^{0}(\mathcal{X})$ - that is, $a \in \omega^{\mathbf{C I}} F(\mathcal{X})$. This proves the lemma.

Notation 4.13. Set $L \in \Phi$. Denote by $s \in S:=\operatorname{Spec} \mathcal{O}_{L}$ the closed point. For all $n \geq 1$ we have $(S, n \cdot s) \in \underline{\mathbf{M C o r}}^{\text {pro }}$ (see Section 3.7). Set $G \in \underline{\mathbf{M P S T}}$; we extend it to a presheaf on $\underline{\mathbf{M C o r}^{\mathrm{pro}}}$. For $n \geq 0$ we introduce the following notation:

$$
G\left(\mathcal{O}_{L}, \mathfrak{m}_{L}^{-n}\right):= \begin{cases}\underline{\omega}_{!} G(S)=G(S, \emptyset) & \text { if } n=0, \\ G(S, n \cdot s) & \text { if } n \geq 1 .\end{cases}
$$

Definition 4.14. Set $F \in \mathbf{R S C}_{\mathrm{Nis}}$ and $G \in \mathbf{C I}(F)$ (see Section 4.9). We denote by $c^{G}=\left\{c_{L}^{G}\right\}$ the family of maps $c_{L}^{G}: F(L) \rightarrow \mathbb{N}_{0}, L \in \Phi$, defined as

$$
c_{L}^{G}(a):=\min \left\{n \geq 0 \mid a \in \tau_{!} G\left(\mathcal{O}_{L}, \mathfrak{m}_{L}^{-n}\right)\right\} .
$$

This is well defined, since

$$
F(L)=\underline{\omega}_{!} \tau_{!}(G)(L)=\tau_{!}(G)(L, \emptyset)=\bigcup_{n} \tau_{!} G\left(\mathcal{O}_{L}, \mathfrak{m}^{-n}\right) .
$$

When $G=\omega^{\mathbf{C I}} F$, we write

$$
c^{F}:=c^{\omega^{\mathbf{C I}} F}
$$

and call $c^{F}$ the motivic conductor of $F$.

Theorem 4.15. Let $F$ be a presheaf with transfers.

(1) If $F$ has a conductor $c$ of some level, then $F \in \mathbf{R S C}$.

(2) If $F \in \mathbf{R S C}_{\mathrm{Nis}}$ and $G \in \mathbf{C I}(F)$ (see Section 4.9), then the family $c^{G}=\left\{c_{L}^{G}\right\}$ (see Definition 4.14) is a conductor for $F$ in the sense of Definition 4.3. In particular, $c^{F}$ is a conductor for $F$.

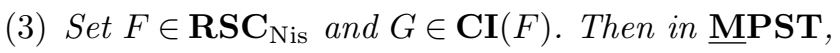

$$
\tau_{!} G \subset \tilde{F}_{c} \text {, }
$$


and for all $L \in \Phi$ and $n \geq 0$, we have

$$
\tau_{!} G\left(\mathcal{O}_{L}, \mathfrak{m}_{L}^{-n}\right)=\tilde{F}_{c^{G}}\left(\mathcal{O}_{L}, \mathfrak{m}_{L}^{-n}\right) .
$$

(4) Set $F \in \mathbf{R S C}_{\mathrm{Nis}}$ and let $c$ be a conductor for $F$ (of some level). Then

$$
\tilde{F}_{c} \subset \tau_{!} \omega^{\mathbf{C I}} F=\tilde{F}_{c^{F}},
$$

where $c^{F}$ is the motivic conductor (see definition (4.14.1)).

In particular,

$$
F \in \mathbf{R S C}_{\mathrm{Nis}} \Longleftrightarrow F \in \mathbf{N S T} \text { and } F \text { has a conductor (of some level). }
$$

Proof. (1). We have $F=\omega_{!} \tau^{*} F_{c} \in \omega_{!}(\mathbf{C I}) \subset \mathbf{R S C}$, by Proposition 4.7 and [27, Proposition 2.3.7]. For (2), we check the properties from Definition 4.3. Set $G_{1}:=\tau_{!} G$; then Definition 4.3, axiom (c1), follows from $\underline{\omega}_{!} G_{1}\left(\mathcal{O}_{L}\right)=\omega_{!} G\left(\mathcal{O}_{L}\right)=F\left(\mathcal{O}_{L}\right)$, and axiom (c2) is obvious. As for axiom (c3), let $L^{\prime} / L$ be a finite extension of henselian dvfs with ramification index $e$. The induced finite morphism $f: \operatorname{Spec} \mathcal{O}_{L^{\prime}} \rightarrow \operatorname{Spec} \mathcal{O}_{L}$ induces a morphism in $\underline{M C o r}^{\text {pro, }}$

$$
\left(\operatorname{Spec} \mathcal{O}_{L}, n \cdot s_{L}\right) \rightarrow\left(\operatorname{Spec} \mathcal{O}_{L^{\prime}}, e n \cdot s_{L^{\prime}}\right),
$$

where $s_{L}$ (resp., $s_{L^{\prime}}$ ) are the closed points. This yields the commutative diagram

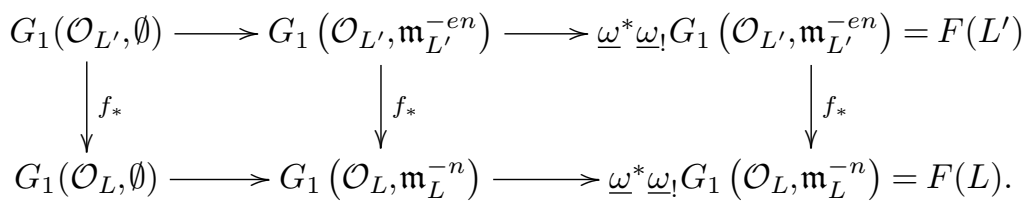

Hence, we obtain the following inequality, which implies axiom (c3):

$$
c_{L}^{G}\left(f_{*} a\right) \leq \min \left\{n \mid a \in G_{1}\left(\mathcal{O}_{L^{\prime}}, \mathfrak{m}_{L^{\prime}}^{-e n}\right)\right\}=\min \left\{n \mid c_{L^{\prime}}^{G}(a) \leq e n\right\} .
$$

The following claim clearly implies axiom (c4):

Claim 4.15.1. Set $X \in \mathbf{S m}$ and $a \in F\left(\mathbf{A}_{X}^{1}\right)$. Assume $X$ connected with function field $K$. Set $K(t)_{\infty}:=\operatorname{Frac}\left(\mathcal{O}_{\mathbf{P}_{K}^{1}, \infty}^{h}\right)$ inducing the henselian dvf point $\operatorname{Spec} K(t)_{\infty} \rightarrow\left(\mathbf{P}_{K}^{1}, \infty\right)$. Assume $c_{K(t)_{\infty}}^{G}\left(a_{K}\right) \leq 1$, where $a_{K} \in F\left(\mathbf{A}_{K}^{1}\right)$ is the restriction of a. Then $a \in F(X)$.

Proof of Claim 4.15.1. The restriction map $F\left(\mathbf{A}_{X}^{1}\right) \rightarrow F\left(\mathbf{A}_{K}^{1}\right)$ is injective, by [26, Theorem 6] and [27, Corollary 3.2.3]; thus it suffices to show $a_{K} \in F(K)$. Set $G_{1, \mathrm{Nis}}:=$ $\underline{a}_{\mathrm{Nis}}\left(G_{1}\right)$ (see Section 3.4). Consider the Nisnevich localisation exact sequence

$$
G_{1, \mathrm{Nis}}\left(\mathbf{P}_{K}^{1}, \infty\right) \rightarrow G_{1, \mathrm{Nis}}\left(\mathbf{A}_{K}^{1}, \emptyset\right) \rightarrow G_{1}\left(K(t)_{\infty}, \emptyset\right) / G_{1}\left(\mathcal{O}_{K(t)_{\infty}}, \infty\right) .
$$

By [45, Theorem 4.1], we have $G_{1, \text { Nis }}\left(\mathbf{A}_{K}^{1}, \emptyset\right)=G_{1}\left(\mathbf{A}_{K}^{1}, \emptyset\right)=F\left(\mathbf{A}_{K}^{1}\right)$. Hence our assumption implies that $a_{K}$ comes from $G_{1, \text { Nis }}\left(\mathbf{P}_{K}^{1}, \infty\right)$ and the desired assertion follows from the cube invariance of $G_{1, N i s}$ (see [45, Theorem 10.1]) and Remark 4.11:

$$
G_{1, \mathrm{Nis}}\left(\mathbf{P}_{K}^{1}, \infty\right) \simeq G_{1, \mathrm{Nis}}(K, \emptyset)=G_{1}(K, \emptyset) \simeq F(K)
$$


Next we prove axiom (c5). Set $X \in \mathbf{S m}$ and $a \in F(X)$. We can assume that $X$ is not proper over $k$. Take any $\mathcal{X}=\left(\bar{X}, X_{\infty}\right) \in$ MCor such that $X=\bar{X}-\left|X_{\infty}\right|$. We have

$$
F(X)=\omega_{!} G(X)=\underset{n>0}{\lim _{n \rightarrow 0}} G\left(\bar{X}, n \cdot X_{\infty}\right),
$$

and hence $a \in G\left(\bar{X}, n \cdot X_{\infty}\right)$, for some $n$. Then for any henselian $\operatorname{dvf}$ point $\operatorname{Spec} L \rightarrow$ $\left(\bar{X}, n \cdot X_{\infty}\right)$, we get $a \in G_{1}\left(\mathcal{O}_{L}, \mathfrak{m}_{L}^{-n v_{L}\left(X_{\infty}\right)}\right)$ so that $c_{L}^{F}(a) \leq n \cdot v_{L}\left(X_{\infty}\right)$. This completes the proof of statement 2 .

For statement (3), it follows directly from the definition of $F_{c^{G}}$ in Proposition 4.7 that $\tau_{!} G \subset F_{c^{G}}$; hence also $\tau_{!} G=\tau_{!} \tau^{*} \tau_{!} G \subset \tau_{!} \tau^{*} F_{c^{G}}=\tilde{F}_{c^{G}}$. Furthermore, the equality in the second part of the statement comes from the inclusions

$$
\tau_{!} G\left(\mathcal{O}_{L}, \mathfrak{m}_{L}^{-n}\right) \subset \tilde{F}_{c^{G}}\left(\mathcal{O}_{L}, \mathfrak{m}_{L}^{-n}\right) \subset\left\{a \in F(L) \mid a \in \tau_{!} G\left(\mathcal{O}_{L}, \mathfrak{m}_{L}^{-n}\right)\right\}
$$

where the first inclusion comes from the foregoing and the second holds by definition. Finally we come to statement (4). The inclusion $\tilde{F}_{c} \subset \tau_{!} \omega^{\mathbf{C I}} F$ follows from Lemma 4.12. The equality $\tilde{F}_{c^{F}}=\tau ! \omega^{\mathbf{C I}} F$ now follows from this and (3). This completes the proof.

Corollary 4.16. The functor $\omega^{\mathbf{C I}}: \mathbf{R S C} \rightarrow \mathbf{C I}$ restricts to a functor $\omega^{\mathbf{C I}}: \mathbf{R S C}_{\mathrm{Nis}} \rightarrow$ $\mathrm{CI}_{\mathrm{Nis}}:=\mathbf{C I} \cap \mathbf{M N S T}$.

Proof. Take $F \in \mathbf{R S C}_{\mathrm{Nis}}$. By Theorem 4.15, Proposition 4.7(3) and formula (4.8.1), we have $\tau_{!} \omega^{\mathbf{C I}} F=\tilde{F}_{c^{F}} \in \underline{\mathbf{M N S T}}$. Hence $\omega^{\mathbf{C I}} F \in \mathbf{M N S T}$, by definition (see Section 3.4).

Notation 4.17. Set $F \in \mathbf{R S C}_{\mathrm{Nis}}$. In the following we will simply write

$$
\tilde{F}:=\tilde{F}_{c^{F}}=\tau ! \omega^{\mathbf{C I}} F .
$$

By Corollary 4.16 we have $\tau^{*} \tilde{F} \in \mathbf{C I}(F)_{\mathrm{Nis}}$ (see Section 4.9$)$.

Corollary 4.18. Set $F \in \mathbf{R S C}_{\text {Nis }}$. Denote by $\left(c^{F}\right)^{\leq n}$ the restriction of the motivic conductor to trdeg $\leq n$. Assume that $\left(c^{F}\right)^{\leq n}$ is a conductor of level $n$. Then

$$
\tilde{F}_{\left(c^{F}\right) \leq n}=\tilde{F} .
$$

Proof. Clearly $\tilde{F}_{c^{F}} \subset \tilde{F}_{\left(c^{F}\right) \leq n}$, and $\supset$ holds by Theorem 4.15(4).

Proposition 4.19. Let $F_{1} \subset F_{2}$ be an inclusion in $\mathbf{R S C}_{\mathrm{Nis}}$. Then the restriction of the motivic conductor of $F_{2}$ to $F_{1}$ is equal to the motivic conductor on $F_{1}$ - that is,

$$
c^{F_{1}}=\left(c^{F_{2}}\right)_{\mid F_{1}} .
$$

Proof. Set $a \in F_{1}(X)$. By the definition of the motivic conductor, it suffices to show that $a$ has modulus $\left(\bar{X}, X_{\infty}\right)$ as an element in $F_{2}(X)$ if and only if it has the same modulus as an element in $F_{1}(X)$. This is obvious (see Definition 3.2). 
Lemma 4.20. Set $F_{1}, F_{2} \in \mathbf{R S C}_{\mathrm{Nis}}$. Set $L \in \Phi$ and $a_{i} \in F_{i}(L)$. Then $c_{L}^{F_{1} \oplus F_{2}}\left(a_{1}+a_{2}\right)=$ $\max \left\{c_{L}^{F_{1}}\left(a_{1}\right), c_{L}^{F_{2}}\left(a_{2}\right)\right\}$.

Proof. This is direct from Definition 4.14.

Proposition 4.21. Let $k_{1} / k$ be an algebraic (hence separable) field extension and set $F \in \mathbf{R S C}_{\mathrm{Nis}, k_{1}}$ (that is, $F$ is a contravariant functor $\mathbf{C o r}_{k_{1}} \rightarrow \mathbf{A b}$ which is a Nisnevich sheaf on $\mathbf{S m}_{k_{1}}$ and has SC-reciprocity). Denote by $R_{k_{1} / k} F: \mathbf{S m}=\mathbf{S m}_{k} \rightarrow \mathbf{A b}$ the functor given by

$$
X \mapsto R_{k_{1} / k} F(X):=F\left(X_{k_{1}}\right),
$$

where $X_{k_{1}}=X \times_{\text {Spec } k} \operatorname{Spec} k_{1}$. Then $R_{k_{1} / k} F \in \mathbf{R S C}_{\mathrm{Nis}}$ and its motivic conductor is given by

$$
c_{L}^{R_{k_{1} / k} F}(a)=\max _{i}\left\{c_{L_{i}}^{F}\left(a_{i}\right)\right\},
$$

where $L \otimes_{k} k_{1} \cong \prod_{i} L_{i}$ and $a=\left(a_{i}\right) \in R_{k_{1} / k} F(L)=\prod_{i} F\left(L_{i}\right)$.

Proof. The first statement follows from the definition of $\mathbf{R} \mathbf{S C}_{\mathrm{Nis}}$; for the second, observe that for $L \in \Phi$ the $k_{1}$-algebra $L \otimes_{k} k_{1}=\prod_{i} L_{i}$ is unramified over $L$, and hence (see Notation 4.17 for notation)

$$
\widetilde{R_{k_{1} / k} F}\left(\mathcal{O}_{L}, \mathfrak{m}_{L}^{-n}\right)=\prod_{i} \tilde{F}\left(\mathcal{O}_{L_{i}}, \mathfrak{m}_{L_{i}}^{-n}\right)
$$

This yields the statement.

\subsection{Semicontinuous conductors}

Definition 4.22. Set $F \in \mathbf{P S T}$ and let $c$ be a conductor of level $n \in[1, \infty]$ for $F$. We say $c$ is semicontinuous if it satisfies the following condition:

(c6) Set $X \in \mathbf{S m}$ with $\operatorname{dim}(X) \leq n$ and $Z \subset X$ a smooth prime divisor with generic point $z$ and $K=\operatorname{Frac}\left(\mathcal{O}_{X, z}^{h}\right)$. Then for any $a \in F(X \backslash Z)$ with $c_{K}\left(a_{K}\right) \leq r$ there exists a Nisnevich neighbourhood $u: U \rightarrow X$ of $z$ and a compactification $\mathcal{Y}=$ $\left(\bar{Y}, Y_{\infty}\right)$ of $\left(U, r \cdot u^{*} Z\right)$ such that (see Definition 4.5 for notation)

$$
c_{\bar{Y}}\left(a_{U}\right) \leq Y_{\infty}
$$

where $a_{U}$ (resp., $a_{K}$ ) denotes the restriction of $a$ to $U$ (resp., $K$ ).

Lemma 4.23. Set $F \in \mathbf{P S T}$ and let $c$ be a conductor of level $n$ for $F$. The following statements are equivalent:

(1) $c$ is semicontinuous;

(2) $\tilde{F}_{c}\left(\mathcal{O}_{L}, \mathfrak{m}_{L}^{-r}\right)=\left\{a \in F(L) \mid c_{L}(a) \leq r\right\}$ for $L \in \Phi_{\leq n}, r \geq 0$.

Proof. Set $a \in F(L)$. Then $a \in \tilde{F}_{c}\left(\mathcal{O}_{L}, \mathfrak{m}_{L}^{-r}\right)$ if and only if there exist a smooth scheme $X$, a smooth prime divisor $Z$ on $X$ with generic point $z$, a $k$-isomorphism $\mathcal{O}_{L} \cong \mathcal{O}_{X, z}^{h}$, 
an element $\tilde{a} \in F(X \backslash Z)$ restricting to $a$ and a compactification $\mathcal{Y}=\left(\bar{Y}, Y_{\infty}\right)$ of $(X, r \cdot Z)$, such that $c_{\bar{Y}}(\tilde{a}) \leq Y_{\infty}$. From this description we see that the $\subset$ inclusion in (2) always holds, while the $\supset$ inclusion is equivalent to the semicontinuity of $c$.

Corollary 4.24. Set $F \in \mathbf{R S C}_{\mathrm{Nis}}$ and let $c$ be a semicontinuous conductor of level $n$ for $F$. Then $\left(c^{F}\right)^{\leq n} \leq c$ - that is, for all $L \in \Phi_{\leq n}$ and all $a \in F(L)$ we have $c_{L}^{F}(a) \leq c_{L}(a)$.

Proof. This follows from Theorem 4.15(4) and Lemma 4.23.

We can summarise part of the foregoing as follows:

Theorem 4.25. Set $F \in \mathbf{R S C}_{\mathrm{Nis}}$.

(1) Any $G \in \mathbf{C I}(F)$ (see Section 4.9) defines a semicontinuous conductor $c^{G}$ (see Definition 4.14). For $G_{1} \subset G_{2}$ in $\mathbf{C I}(F)$, we have $c^{G_{2}} \leq c^{G_{1}}$.

(2) Let c be a conductor of level $n \in[0, \infty]$. Then $\tau^{*} F_{c} \in \mathbf{C I}(F)_{\mathrm{Nis}}$. For $c_{1} \leq c_{2}$ we have $\tau^{*} F_{c_{2}} \subset \tau^{*} F_{c_{1}}$. If, furthermore, $c$ is semicontinuous, then $c=\left(c^{\tau^{*} F_{c}}\right)^{\leq n}$.

(3) Assume that $G \in \mathbf{C I}(F)_{\mathrm{Nis}}$ satisfies

$$
G(\mathcal{X})=\left\{a \in F(X) \mid \rho^{*} a \in \tau_{!} G\left(\mathcal{O}_{L}, \mathfrak{m}_{L}^{-v_{L}\left(X_{\infty}\right)}\right), \quad \begin{array}{l}
\forall \rho: \operatorname{Spec} L \rightarrow \mathcal{X}, \\
\text { with } L \in \Phi
\end{array}\right\},
$$

for all proper modulus pairs $\mathcal{X}=\left(\bar{X}, X_{\infty}\right)$ with $X=\bar{X} \backslash\left|X_{\infty}\right|$. Then $\tau^{*} F_{c^{G}}=G$.

(4) Let $c$ be a semicontinuous conductor of level $n$ for $F$ (possibly only defined on $\operatorname{trdeg} \leq n)$. Then there exists a unique semicontinuous conductor $c^{\infty}$ for $F$ with the properties

$$
\tau^{*} F_{c}=\tau^{*} F_{c^{\infty}} \quad \text { and } \quad c=\left(c^{\infty}\right)^{\leq n} .
$$

We call $c^{\infty}$ the canonical extension of $c$.

(5) Assume that the restriction $\left(c^{F}\right)^{\leq n}$ of the motivic conductor to trdeg $\leq n$ is a conductor. Then its canonical extension is the motivic conductor - that is, $\left(\left(c^{F}\right)^{\leq n}\right)^{\infty}=c^{F}$.

Proof. (1) holds from Theorem 4.15(3) and Lemma 4.23. (2) follows from Lemmas 4.12 and 4.23. (3) holds by the definitions involved. For (4), set $G:=\tau^{*} F_{c}$; then $G \in \mathbf{C I}(F)$ by (2) and it satisfies the condition from (3) by Lemma 4.23. Set $c^{\infty}:=c^{G}$. Then $c^{\infty}$ has the desired properties by (3) and (2). Finally, (5) follows from Corollary 4.18.

We finish this section with some lemmas which are needed later.

Definition 4.26. Set $F \in \mathbf{R S C}_{\mathrm{Nis}}$. We say $F$ is proper if the following equivalent conditions are satisfied:

(1) For all $X \in \mathbf{S m}$ and any dense open $U \subset X$, the restriction map $F(X) \stackrel{\simeq}{\rightarrow} F(U)$ is an isomorphism.

(2) Any conductor $c$ on $F$ is trivial - that is, $c_{L}=0$ for all $L$. 
For $(2) \Rightarrow(1)$, use the fact that axiom (c4) implies $F \in \mathbf{H I}_{\mathrm{Nis}}$, and then the statement follows from Voevodsky's Gersten resolution (compare [28, Lemma 10.3]).

Lemma 4.27. Let $0 \rightarrow F_{1} \stackrel{\varphi}{\rightarrow} F \stackrel{\psi}{\rightarrow} F_{2} \rightarrow 0$ be an exact sequence in NST and with $F_{1}$, $F_{2} \in \mathbf{R S C}_{\mathrm{Nis}}$, and assume $F_{1}$ is proper.

Then $F \in \mathbf{R S C}_{\mathrm{Nis}}$. Any (semicontinuous) conductor $c$ of level $n$ on $F_{2}$ induces a (semicontinuous) conductor $c \psi=\left\{c_{L} \circ \psi\right\}_{L}$ of level $n$ on $F$. Furthermore, the motivic conductor of $F$ is given by $c^{F}=c^{F_{2}} \psi$.

Proof. Let $c$ be a conductor of level $n$ on $F_{2}$. Then $c \psi$ clearly satisfies axioms (c2), (c3) and (c5) (and (c6), if $c$ does). By the properness of $F_{1}$, we have an isomorphism $F(L) / F\left(\mathcal{O}_{L}\right) \cong F_{2}(L) / F_{2}\left(\mathcal{O}_{L}\right)$, which implies $(\mathrm{c} 1)$. Assume that $a \in F\left(\mathbf{A}_{X}^{1}\right)$ satisfies the assumption in (c4) for $c \psi$. Let $\pi: \mathbf{A}_{X}^{1} \rightarrow X$ be the projection and $i: X \hookrightarrow \mathbf{A}_{X}^{1}$ the zero section. Then $\psi\left(a-\pi^{*} i^{*} a\right)=\psi(a)-\pi^{*} i^{*} \psi(a) \in F_{2}\left(\mathbf{A}_{X}^{1}\right)$ satisfies the assumption from (c4) for $c$; hence it lies in $\pi^{*} F_{2}(X)$, and thus is zero. Therefore, $a-\pi^{*} i^{*} a \in F_{1}\left(\mathbf{A}_{X}^{1}\right)=\pi^{*} F_{1}(X)$, and hence it is zero - that is, $a=\pi^{*} i^{*} a$. This shows that $c \psi$ satisfies $(c 4)$. Therefore $c \psi$ is a conductor of level $n$. Thus Theorem 4.15 yields $F \in \mathbf{R S C}_{\mathrm{Nis}}$ and $\tilde{F}_{c^{F_{2}}}\left(\mathcal{O}_{L}, \mathfrak{m}_{L}^{-n}\right) \subset$ $\tilde{F}\left(\mathcal{O}_{L}, \mathfrak{m}_{L}^{-n}\right)$. We have inclusions

$$
\tilde{F}_{c^{F_{2}} \psi}\left(\mathcal{O}_{L}, \mathfrak{m}_{L}^{-n}\right) / F_{1}\left(\mathcal{O}_{L}\right) \hookrightarrow \tilde{F}\left(\mathcal{O}_{L}, \mathfrak{m}_{L}^{-n}\right) / F_{1}\left(\mathcal{O}_{L}\right) \hookrightarrow \tilde{F}_{2}\left(\mathcal{O}_{L}, \mathfrak{m}_{L}^{-n}\right),
$$

where the second map is injective by the properness of $F_{1}$. Since $\tilde{F}_{2}=\tilde{F}_{2, c^{F_{2}}}$, the composition is an isomorphism; thus $c^{F}=c^{F_{2}} \psi$.

Lemma 4.28. Let $\varphi: F \rightarrow G$ be a surjection in NST. Let $c=\left\{c_{L}: F(L) \rightarrow \mathbb{N}\right\}_{L \in \Phi_{\leq n}}$ be a collection of maps. Define $\bar{c}=\left\{\bar{c}_{L}: G(L) \rightarrow \mathbb{N}_{L \in \Phi_{\leq n}}\right.$ by

$$
\bar{c}_{L}(a):=\min \left\{c_{L}(\tilde{a}) \mid \tilde{a} \in F(L) \text { with } \varphi(\tilde{a})=a\right\} .
$$

If c satisfies (c1) (resp., (c2), (c3) or (c6)), then so does $\bar{c}$.

Furthermore, suppose $\varphi$ has the following property: for all $X \in \mathbf{S m}$ there exists a proper modulus pair $\left(\bar{X}, X_{\infty}\right)$ with $X=\bar{X} \backslash X_{\infty}$, such that for all $x \in \bar{X}$ the map $\varphi$ induces a surjection

$$
F\left(\bar{X}_{(x)}^{h} \backslash X_{\infty,(x)}\right) \rightarrow G\left(\bar{X}_{(x)}^{h} \backslash X_{\infty,(x)}\right)
$$

where $\bar{X}_{(x)}^{h}=\operatorname{Spec} \mathcal{O} \frac{h}{X, x}$ and $X_{\infty,(x)}$ denotes the restriction of $X_{\infty}$ to $\bar{X}_{(x)}^{h}$. Then $\bar{c}$ satisfies (c5), if c does.

Proof. (c1). If $\bar{c}_{L}(a)=0$, then there exists a lift $\tilde{a} \in F(L)$ with $c_{L}(\tilde{a})=0$, and hence $\tilde{a} \in F\left(\mathcal{O}_{L}\right)$ by $(c 1)$ for $c$. Therefore $a \in G\left(\mathcal{O}_{L}\right)$.

(c2). Set $a, b \in G(L)$. Take lifts $\tilde{a}, \tilde{b} \in F(L)$ with $c_{L}(\tilde{a})=\bar{c}_{L}(a)$ and $c_{L}(\tilde{b})=\bar{c}_{L}(b)$. Then by (c2) for $c$,

$$
\bar{c}_{L}(a+b) \leq c_{L}(\tilde{a}+\tilde{b}) \leq \max \left\{c_{L}(\tilde{a}), c_{L}(\tilde{b})\right\}=\max \left\{\bar{c}_{L}(a), \bar{c}_{L}(b)\right\}
$$


(c3). Let $f: \operatorname{Spec} L^{\prime} \rightarrow \operatorname{Spec} L$ be a finite extension with ramification index $e$ and set $a \in G\left(L^{\prime}\right)$. Take a lift $\tilde{a} \in F\left(L^{\prime}\right)$ with $\bar{c}_{L^{\prime}}(a)=c_{L^{\prime}}(\tilde{a})$. Then by $(\mathrm{c} 3)$ for $c$,

$$
\bar{c}_{L}\left(f_{*} a\right) \leq c_{L}\left(f_{*} \tilde{a}\right) \leq\left\lceil\frac{c_{L^{\prime}}(\tilde{a})}{e}\right\rceil=\left\lceil\frac{\bar{c}_{L^{\prime}}(a)}{e}\right\rceil .
$$

(c6). Let $X, z \in Z, K$ be as in (c6) and $a \in G(X \backslash Z)$ with $\bar{c}_{K}\left(a_{K}\right) \leq r$. Let $\tilde{a}_{K} \in F(K)$ be a lift of $a_{K}$ with $c_{K}\left(\tilde{a}_{K}\right)=\bar{c}_{K}\left(a_{K}\right)$. Since $\operatorname{Spec} K=\operatorname{Spec} \mathcal{O}_{K} \backslash Z_{\mathcal{O}_{K}}$, we find a Nisnevich neighbourhood $U \rightarrow X$ of $z$ and an element $\tilde{a} \in F(U \backslash Z)$ which restricts to $\tilde{a}_{K}$. After possibly shrinking $U$ around $z$, we may assume that $\varphi(\tilde{a})=a_{\mid U \backslash Z_{U}}$. By (c6) for $F$, we may shrink $U$ further around $z$ to obtain a compactification $\mathcal{Y}=\left(\bar{Y}, Y_{\infty}\right)$ of $\left(U, r \cdot Z_{U}\right)$ such that

$$
\bar{c}_{\bar{Y}}\left(a_{U}\right) \leq c_{\bar{Y}}\left(\tilde{a}_{U}\right) \leq Y_{\infty}
$$

(c5). Assuming condition (4.28.1), set $X \in \mathbf{S m}$ and $a \in G(X)$. Let $\mathcal{X}=\left(\bar{X}, X_{\infty}\right)$ be a proper modulus pair with $X=\bar{X} \backslash\left|X_{\infty}\right|$ as in condition (4.28.1). This condition implies that we find a finite Nisnevich cover $\left\{U_{i} \rightarrow \bar{X}\right\}_{i}$ and $\tilde{a}_{i} \in F\left(U_{i, X}\right)$ such that $\varphi\left(\tilde{a}_{i}\right)=a_{\mid U_{i, X}}$ in $G\left(U_{i, X}\right)$, where $\left\{U_{i, X} \rightarrow X\right\}_{i}$ is the induced Nisnevich cover of $X$. Let $\mathcal{Y}_{i}=\left(\bar{Y}_{i}, Y_{i, \infty}\right)$ be a compactification of $\left(U_{i}, X_{\infty \mid U_{i}}\right)$ which admits a morphism $\overline{Y_{i}} \rightarrow \bar{X}$ extending $U_{i} \rightarrow$ $\bar{X}$ and inducing a morphism of proper modulus pairs $\mathcal{Y}_{i} \rightarrow \mathcal{X}$. By (c5) for $c$ and (the proof of Lemma 4.6, we find an integer $N \gg 0$ such that $c_{L}\left(\rho^{*} \tilde{a}_{i}\right) \leq N \cdot v_{L}\left(Y_{i, \infty}\right)$ for all $\rho:$ Spec $L \rightarrow U_{i, X}=\bar{Y}_{i} \backslash\left|Y_{i, \infty}\right|, L \in \Phi_{\leq n}$. Let $\rho: \operatorname{Spec} L \rightarrow X$ be any henselian dvf point with $L \in \Phi_{\leq n}$; denote by $s \in \bar{X}$ the image of the closed point under the induced map $\bar{\rho}: \operatorname{Spec}_{\mathcal{O}_{L}} \rightarrow \bar{X}$. By the Nisnevich property, there exist an $i$ and a point $s_{i} \in U_{i}$ such that $U_{i} \rightarrow \bar{X}$ induces an isomorphism $s_{i} \stackrel{\simeq}{\rightarrow} s$. Hence $\bar{\rho}$ factors via $U_{i} \hookrightarrow \bar{Y}_{i} \rightarrow \bar{X}$. Thus

$$
\bar{c}_{L}\left(\rho^{*} a\right) \leq c_{L}\left(\rho^{*} \tilde{a}_{i}\right) \leq N \cdot v_{L}\left(Y_{i, \infty}\right)=N \cdot v_{L}\left(X_{\infty}\right),
$$

where for the equality we used $\left(Y_{i, \infty}\right)_{\mid U_{i}}=\left(X_{\infty}\right)_{\mid U_{i}}$. Thus a satisfies (c5) for $\left(\bar{X}, N \cdot X_{\infty}\right)$.

\subsection{Homotopy-invariant subsheaves}

Corollary 4.29. Let $F \in \mathbf{N S T}$ be $\mathbf{A}^{1}$-invariant (in particular, $F \in \mathbf{R S C}_{\mathrm{Nis}}$ ). Then the motivic conductor of $F$ is given by

$$
c_{L}^{F}(a)= \begin{cases}0 & \text { if } a \in F\left(\mathcal{O}_{L}\right), \\ 1 & \text { otherwise }\end{cases}
$$

Proof. The right-hand side defines a conductor, as already noted in Remark 4.4; it is clearly semicontinuous. By Corollary 4.24 we get $\leq$ in the statement, and (c1) forces it to be an equality.

4.30. We denote by $\mathbf{H I}$ the category of $\mathbf{A}^{1}$-invariant presheaves with transfers and set $\mathbf{H I}_{\text {Nis }}:=\mathbf{H I} \cap \mathbf{N S T}$. It follows immediately from Definition 3.2 that we have $\mathbf{H I} \subset \mathbf{R S C}$ and $\mathbf{H I}_{\mathrm{Nis}} \subset \mathbf{R S C}_{\mathrm{Nis}}$. 
Set $F \in \mathbf{P S T}$. For $X \in \mathbf{S m}$, we denote by

$$
h_{\mathbf{A}^{1}}^{0}(F)(X)
$$

the subset of $F(X)$ formed by those sections $a \in F(X)$ for which the Yoneda map $a$ : $\mathbb{Z}_{\mathrm{tr}}(X) \rightarrow F$ factors via

$$
h_{0}^{\mathbf{A}^{1}}(X)=\operatorname{Coker}\left(\mathbb{Z}_{\mathrm{tr}}(X)\left(-\times \mathbf{A}_{k}^{1}\right) \stackrel{i_{0}^{*}-i_{1}^{*}}{\longrightarrow} \mathbb{Z}_{\mathrm{tr}}(X)\right) \in \mathbf{P S T} .
$$

We immediately see that $X \mapsto h_{\mathbf{A}^{1}}^{0}(F)(X)$ defines a subpresheaf with transfers of $F$, since $h_{0}^{\mathbf{A}^{1}}(X) \in \mathbf{H I}$ (see, for example, [48, Proposition 3.6]) and we have $h_{\mathbf{A}^{1}}^{0}(F) \in \mathbf{H I}$; furthermore, it has the universal property that any morphism $H \rightarrow F$ in PST with $H \in \mathbf{H I}$ factors uniquely via a morphism $H \rightarrow h_{\mathbf{A}^{1}}^{0}(F)$ in $\mathbf{H I}$. Note that if $F \in \mathbf{N S T}$, then $h_{\mathbf{A}^{1}}^{0}(F) \in \mathbf{H I}_{\mathrm{Nis}}$. Indeed, by [49, Theorem 3.1.12], Nisnevich sheafification induces an exact functor $\mathbf{H I} \rightarrow \mathbf{H I}_{\mathrm{Nis}}$, and thus we obtain natural inclusions in PST

$$
h_{\mathbf{A}^{1}}^{0}(F) \hookrightarrow h_{\mathbf{A}^{1}}^{0}(F)_{\mathrm{Nis}} \hookrightarrow F_{\mathrm{Nis}}=F .
$$

Since $h_{\mathbf{A}^{1}}^{0}(F)_{\text {Nis }} \in \mathbf{H I}$, the second inclusion factors via $h_{\mathbf{A}^{1}}^{0}(F)$; hence $h_{\mathbf{A}^{1}}^{0}(F)=$ $h_{\mathbf{A}^{1}}^{0}(F)_{\mathrm{Nis}}$.

Proposition 4.31. Set $F \in \mathbf{P S T}$ and let $c$ be a conductor of level $n$ for $F$. Then

$$
X \mapsto F^{c \leq 1}(X):=\left\{a \in F(X) \mid c_{L}\left(\rho^{*} a\right) \leq 1, \quad \begin{array}{l}
\forall \rho: \operatorname{Spec} L \rightarrow X \\
\text { with } L \in \Phi_{\leq n}
\end{array}\right\}
$$

defines a homotopy-invariant subpresheaf with transfers of $F$. If $F \in \mathbf{N S T}$, then $F^{c \leq 1} \in$ $\mathbf{H I}_{\mathrm{Nis}}$.

Proof. Showing that $F^{c \leq 1} \in \mathbf{P S T}$ is equivalent to the following: let $V \in \operatorname{Cor}(X, Y)$ be a finite prime correspondence and set $a \in F^{c \leq 1}(Y)$; then for all henselian dvf points $\rho: \operatorname{Spec} L \rightarrow X$ with $\operatorname{trdeg}(L / k) \leq n$, we have

$$
c_{L}\left(\rho^{*} V^{*} a\right) \leq 1
$$

This follows from the calculation in equation (4.7.4). The $\mathbf{A}^{1}$-invariance of $F^{c \leq 1}$ follows directly from (c4). The last statement is proven similarly as in Proposition 4.7(3).

Corollary 4.32. Set $F \in \mathbf{R S C}_{\mathrm{Nis}}$ with motivic conductor $c^{F}$. Then

$$
h_{\mathbf{A}^{1}}^{0}(F)=F^{c^{F} \leq 1} .
$$

Proof. By Proposition 4.31 we have $F^{c^{F} \leq 1} \subset h_{\mathbf{A}^{1}}^{0}(F)$. By Proposition 4.19 and Corollary 4.29 we have $\left(c^{F}\right)_{\mid h_{\mathbf{A}^{1}}^{0}(F)}=c^{h_{\mathbf{A}^{1}}^{0}(F)} \leq 1$; hence $h_{\mathbf{A}^{1}}^{0}(F) \subset F^{c^{F} \leq 1}$.

Corollary 4.33. Set $F \in \mathbf{R S C}_{\mathrm{Nis}}$. Assume that for all $L \in \Phi$ we have

$$
\tilde{F}\left(\mathcal{O}_{L}, \mathfrak{m}_{L}^{-1}\right)=F\left(\mathcal{O}_{L}\right)
$$


Let $X \in \mathbf{S m}$ be proper over $k$ and $U \subset X$ be dense open. Then

$$
h_{\mathbf{A}^{1}}^{0}(F)(U)=F(X) .
$$

In particular, if $F$ satisfies equation (4.33.1), then $X \mapsto F(X)$ is a birational invariant on smooth proper schemes.

Proof. By Corollary 4.32,

$$
h_{\mathbf{A}^{1}}^{0}(F)(U)=F^{c^{F} \leq 1}(U) .
$$

Hence $F(X) \subset h_{\mathbf{A}^{1}}^{0}(F)(U)$, and by equation (4.33.1) we also have

$$
h_{\mathbf{A}^{1}}^{0}(F)(U) \subset \bigcap_{x \in X^{(1)}} F\left(\mathcal{O}_{X, x}^{h}\right) .
$$

By [45, Cor 0.3],

$$
\bigcap_{x \in X^{(1)}} F\left(\mathcal{O}_{X, x}^{h}\right)=F(X) .
$$

Taking all this together yields the statement.

\subsection{Local symbols}

4.34. We recall the notion of local symbols for reciprocity sheaves; see [47, III, §1], [26, Proposition 5.2.1] or [23, 1.5] for details.

Set $F \in \mathbf{R S C}_{\text {Nis }}$. If $L / K$ is a finite field extension of finitely generated fields over $k$, we denote by $\operatorname{Tr}_{L / K}: F(L) \rightarrow F(K)$ the map induced by the transfer structure on $F$. For $X \in$ Cor $^{\text {pro }}, x \in X$ and $a \in F(X)$ we denote by $a(x) \in F(x)$ the pullback of $a$ along $x \hookrightarrow X$.

Let $K$ be a function field over $k$ and $C$ be a regular projective $K$-curve. Note that $C \in \mathbf{C o r}^{\text {pro }}$, by Lemma 2.4. For $x \in C_{(0)}$ a closed point, we write $v_{x}$ for the corresponding normalised discrete valuation on $K(C)^{\times}$and $\mathfrak{m}_{x} \subset \mathcal{O}_{C, x}$ for the maximal ideal, and set $U_{x}^{(n)}:=1+\mathfrak{m}_{x}^{n} \subset \mathcal{O}_{C, x}^{\times}, n \geq 1$. Let $D=\sum n_{x} \cdot x$ be an effective Cartier divisor on $C$ and set $a \in \tilde{F}(C, D)$ (see Notation 4.17 for the notation $\tilde{F}$ ). Then there exists a family of maps

$$
\left\{(a,-)_{C / K, x}: K(C)^{\times} \rightarrow F(K)\right\}_{x \in C_{(0)}}
$$

which is uniquely determined by the following properties:

(LS1) $(a,-)_{C / K, x}: K(C)^{\times} \rightarrow F(K)$ is a group homomorphism;

(LS2) $(a, f)_{C / K, x}=v_{x}(f) \operatorname{Tr}_{K(x) / K}(a(x))$, for $x \in C \backslash|D|$;

$(\mathrm{LS} 3)\left(a, U_{x}^{\left(n_{x}\right)}\right)_{C / K, x}=0$

(LS4) $\sum_{x \in C_{(0)}}(a, f)_{C / K, x}=0$.

It follows from uniqueness that the family $\left\{(a,-)_{C / K, x}\right\}$ does not depend on the chosen modulus $D$. Furthermore, from uniqueness we can deduce the following properties:

$(\mathrm{LS} 5)(-,-)_{C / K, x}: F(K(C)) \times K(C)^{\times} \rightarrow F(K)$ is bilinear; 
(LS6) if $h: F \rightarrow G$ is a morphism in $\mathbf{R S C}_{\mathrm{Nis}}$, then in $G(K)$,

$$
h\left((a, f)_{C / K, x}\right)=(h(a), f)_{C / K, x}, \quad \text { all } a \in F(K(C)), f \in K(C)^{\times} .
$$

Let $K^{\prime} / K$ be a finite field extension, $C^{\prime} / K^{\prime} \in$ Cor $^{\text {pro }}$ a projective curve and $\pi: C^{\prime} \rightarrow C$ a finite morphism over $\operatorname{Spec} K^{\prime} \rightarrow \operatorname{Spec} K$. Then we have the following properties:

(LS7) for $b \in F\left(K^{\prime}\left(C^{\prime}\right)\right), f \in K(C)^{\times}$and $x \in C_{(0)}$,

$$
\left(\pi_{*}(b), f\right)_{C / K, x}=\sum_{y / x} \operatorname{Tr}_{K^{\prime} / K}\left(b, \pi^{*} f\right)_{C^{\prime} / K^{\prime}, y}
$$

(LS8) for $a \in F(K(C)), g \in K^{\prime}\left(C^{\prime}\right)^{\times}$and $x \in C_{(0)}$,

$$
\left(a, \pi_{*} g\right)_{C / K, x}=\sum_{y / x} \operatorname{Tr}_{K^{\prime} / K}\left(\pi^{*}(a), g\right)_{C^{\prime} / K^{\prime}, y} .
$$

In both cases, the sum is over all $y \in C^{\prime}$ mapping to $x$.

Lemma 4.35. Set $F \in \mathbf{R S C}_{\mathrm{Nis}}$ and let $C$ be a regular projective and geometrically connected $K$-curve. Let $K^{\prime} / K$ be a finitely generated field extension, and denote by $\tau: \operatorname{Spec} K^{\prime} \rightarrow \operatorname{Spec} K$ the induced map and by $\tau_{C}: C_{K^{\prime}}=C \otimes_{K} K^{\prime} \rightarrow C$ the projection. Then

$$
\sum_{y \in \tau_{C}^{-1}(x)}\left(\tau_{C}^{*} a, \tau_{C}^{*} f\right)_{C_{K^{\prime}} / K^{\prime}, y}=\tau^{*}(a, f)_{C / K, x} \quad \text { in } F\left(K^{\prime}\right)
$$

for all $a \in F(K(C)), f \in K(C)^{\times}$and $x \in C_{(0)}$.

Proof. Let $U \subset C$ be open with $a \in F(U)$. Using the approximation lemma, (LS1) and (LS3), we can assume that for a given $m \geq 1$ we have $f \in U_{z}^{(m)}$ for all $z \in C \backslash(U \cup\{x\})$; in particular, choosing $m$ large enough we get $(a, f)_{C / K, z}=0$. Identifying $f$ with the finite $K$-morphism $C \rightarrow \mathbf{P}_{K}^{1}$, we obtain $a \in F\left(f^{-1}\left(\mathbf{P}_{K}^{1} \backslash\{1\}\right) \backslash\{x\}\right)$, and (LS2) and (LS4) yield

$$
-(a, f)_{C / K, x}=\left(i_{0}^{*}-i_{\infty}^{*}\right) f_{*} a \text { in } F(K) .
$$

The formula in the statement now follows by applying $\tau^{*}$ to this equality, using the base-change formula $\tau_{\mathbf{P}^{1}}^{*} \circ f_{*}=\left(\tau_{C}^{*} f\right)_{*} \tau_{C}^{*}$ induced by the cartesian diagram

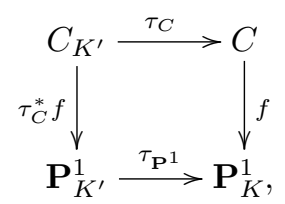

and using (LS1)-(LS4) backwards.

Lemma 4.36. Set $L \in \Phi$. Let $C$ be a regular curve over a $k$-function field $K$. Assume that there exist a closed point $x \in C$ and a $k$-morphism $u: \operatorname{Spec} \mathcal{O}_{L} \rightarrow C$ inducing an isomorphism $\mathcal{O}_{C, x}^{h} \cong \mathcal{O}_{L}$. Then there is an isomorphism

$$
F(K(C)) / F\left(\mathcal{O}_{C, x}\right) \stackrel{\simeq}{\longrightarrow} F(L) / F\left(\mathcal{O}_{L}\right)
$$


induced via pullback along $u$. If $\mathcal{O}_{C, x}$ has a coefficient field, then we have an isomorphism

$$
F(K(C)) / F\left(\mathcal{O}_{C, x}, \mathfrak{m}\right) \stackrel{\simeq}{\rightarrow} F(L) / F\left(\mathcal{O}_{L}, \mathfrak{m}\right),
$$

where for a local ring $A \in \mathbf{C o r}^{\text {pro }}$ with maximal ideal $\mathfrak{m}$ we set

$$
F(A, \mathfrak{m}):=\operatorname{Ker}(F(A) \rightarrow F(A / \mathfrak{m})) .
$$

Proof. We prove the first isomorphism. The natural map in the statement is compatible with pullbacks and push-forwards on both sides. Thus we can apply the standard trick replacing $k$ by its maximal pro- $\ell$ extensions for various primes $\ell$, to assume $k$ is infinite. By Gabber's presentation theorem (see, for example, [8, 3.1.2]), we find an open $U \subset C$ containing $x$, a $k$-function field $E$ and an étale morphism $\varphi: U \rightarrow \mathbf{P}_{E}^{1}$ such that $x=\varphi^{-1}(\varphi(x))$ and $\varphi$ induces an isomorphism $x \stackrel{\simeq}{\rightarrow} \varphi(x)$. It follows from [45, Lemma 4.2, Lemma 4.3] that $(U, n \cdot x)$ is a $V$-pair, for all $n \geq 1$, in the sense of [45, Definition 2.1]. If $v: U^{\prime} \rightarrow U$ is an affine Nisnevich neighbourhood of $x$ with $v^{-1}(x)=\left\{x^{\prime}\right\}$, then the pullback $v^{*}: F(K(U)) / F\left(\mathcal{O}_{U, x}\right) \stackrel{\simeq}{\rightarrow} F\left(K\left(U^{\prime}\right)\right) / F\left(\mathcal{O}_{U^{\prime}, x,}\right)$ is an isomorphism, by [45, Lemma 4.4, (3)]. We obtain the first isomorphism of the statement by taking the limit over all Nisnevich neighbourhoods $v$. For the second isomorphism, observe that if a coefficient field $\sigma: \kappa \hookrightarrow \mathcal{O}_{C, x}$ exists, then $\sigma^{*}$ induces a splitting of the restriction to the closed point $F\left(\mathcal{O}_{C, x}\right) \rightarrow F(\kappa)$; in particular, it is surjective. We obtain isomorphisms $F\left(\mathcal{O}_{C, x}\right) / F\left(\mathcal{O}_{C, x}, \mathfrak{m}_{x}\right) \cong F(\kappa) \cong F\left(\mathcal{O}_{L}\right) / F\left(\mathcal{O}_{L}, \mathfrak{m}_{L}\right)$ which together with the first statement and the five lemma yield the second isomorphism in the statement.

4.37. Set $F \in \mathbf{R S C}_{\mathrm{Nis}}$. Let $L \in \Phi$ have residue field $\kappa=\mathcal{O}_{L} / \mathfrak{m}_{L}$, and let $\sigma: K \hookrightarrow \mathcal{O}_{L}$ be a $k$-homomorphism such that the induced map $K \hookrightarrow \kappa$ is a finite field extension (for example, $\sigma$ could be a coefficient field.) We define the local symbol

$$
(-,-)_{L, \sigma}: F(L) \times L^{\times} \rightarrow F(K)
$$

as follows: we find a regular projective $K$-curve $C$ and a $\kappa$-point $x \in C(\kappa)$ satisfying

$$
L=\operatorname{Frac}\left(\mathcal{O}_{C, x}^{h}\right), \quad \sigma: K \rightarrow \mathcal{O}_{C, x} \stackrel{\text { nat. }}{\longrightarrow} \mathcal{O}_{L} .
$$

Additionally, we assume that $\mathcal{O}_{C, x}$ has a coefficient field. Denote by $u: \operatorname{Spec} \mathcal{O}_{L} \rightarrow$ $\operatorname{Spec} \mathcal{O}_{C, x}$ the induced map. The symbol $(-,-)_{L, \sigma}$ is defined as the composition

$$
\begin{aligned}
& (-,-)_{L, \sigma}: F(L) \times L^{\times} \longrightarrow F(L) / F\left(\mathcal{O}_{L}, \mathfrak{m}_{L}\right) \times{\underset{n}{\lim }}_{L^{\times}} / U_{L}^{(n)}
\end{aligned}
$$

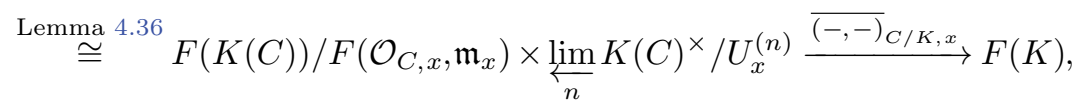

where the last map is given by

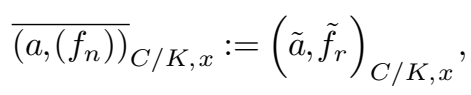

with $\tilde{a} \in \tilde{F}\left(\mathcal{O}_{C, x}, \mathfrak{m}^{-r}\right)$ a lift of $a$ and $\tilde{f}_{r} \in K(C)^{\times}$a lift of $f_{r}$; this is well defined and bilinear by (LS2), (LS3) and (LS5). 
Lemma 4.38. The symbol $(-,-)_{L, \sigma}$ defined in Section 4.37 is independent of the choice of the presentation (4.37.1).

Proof. Let $v: C^{\prime} \rightarrow C$ be a $K$-morphism between regular projective $K$-curves, and let $x \in C$ and $x^{\prime} \in C^{\prime}$ be closed points such that $v$ is étale in a neighbourhood of $x^{\prime}$ and induces an isomorphism $x^{\prime} \stackrel{\simeq}{\longrightarrow} x$. Assume that $\mathcal{O}_{C, x}$ has a coefficient field. Let $E=K(C)$ and $E^{\prime}=K\left(C^{\prime}\right)$ be the function fields. Then it suffices to show that for all $a \in F(E)$ and $f \in E^{\times}$, we have

$$
(a, f)_{C / K, x}=\left(v^{*} a, v^{*} f\right)_{C^{\prime} / K, x^{\prime}}
$$

We denote $E_{x}^{\times}:=\lim _{\longleftarrow} E^{\times} / U_{x}^{(n)}$, and so on. Then the composition

$$
E_{x}^{\times} \stackrel{1 \otimes \mathrm{id}}{\longrightarrow}\left(E_{x} \otimes_{E} E^{\prime}\right)^{\times} \cong \prod_{y / x} E_{y}^{\prime \times} \stackrel{\text { proj }}{\longrightarrow} E_{x^{\prime}}^{\prime \times}
$$

is induced by $v^{*}$ and is an isomorphism with its inverse induced by the norm. Thus we can use the approximation lemma, (LS3) and the continuity of the norm map to choose $g \in E^{\prime \times}$ close to $v^{*} f$ at $x^{\prime}$ and close to 1 at all $y \in v^{-1}(x) \backslash\left\{x^{\prime}\right\}$ to obtain the following:

(1) $\left(v^{*} a, v^{*} f\right)_{C^{\prime} / K, x^{\prime}}=\left(v^{*} a, g\right)_{C^{\prime} / K, x^{\prime}}$;

(2) $\left(v^{*} a, g\right)_{C^{\prime} / K, y^{\prime}}=0$ for all $y^{\prime} \in v^{-1}(x) \backslash\left\{x^{\prime}\right\}$;

(3) $(a, f)_{C / K, x}=\left(a, \mathrm{Nm}_{E^{\prime} / E}(g)\right)_{C / K, x}$.

We thus have

$$
(a, f)_{C / K, x} \stackrel{(\mathrm{LS} 8),(3)}{=} \sum_{y^{\prime} \in v^{-1}(x)}\left(v^{*} a, g\right)_{C^{\prime} / K, y^{\prime}} \stackrel{(1),(2)}{=}\left(v^{*} a, v^{*} f\right)_{C^{\prime} / K, x^{\prime}}
$$

which yields the statement.

Remark 4.39. Note that if the composition $K \stackrel{\sigma}{\rightarrow} \mathcal{O}_{L} \rightarrow \kappa$ is purely inseparable, then there does not need to exist a coefficient field of $\mathcal{O}_{L}$ which contains $K$. This is why in Section 4.37 it does not in general suffice to consider coefficient fields. (In characteristic 0 it does.) For coefficient fields $\sigma: K \hookrightarrow \mathcal{O}_{L}$, the symbol $(-,-)_{L, \sigma}$ will in general depend on the choice of $\sigma$.

Corollary 4.40. Let $L_{1} / L$ be an extension of henselian dvfs of ramification index $e-$ that is, $\mathfrak{m}_{L} \mathcal{O}_{L_{1}}=\mathfrak{m}_{L_{1}}^{e}$. (The extension $L_{1} / L$ does not need to be algebraic or finitely generated.) Let $\sigma_{1}: K \rightarrow \mathcal{O}_{L_{1}}$ be a $k$-homomorphism inducing a finite field extension $K \hookrightarrow \mathcal{O}_{L_{1}} / \mathfrak{m}_{L_{1}}$. Set $F \in \mathbf{R S C}_{\mathrm{Nis}}$ and $a \in \tilde{F}\left(\mathcal{O}_{L}, \mathfrak{m}_{L}^{-r}\right), r \geq 0$. Then

$$
\left(a_{L_{1}}, U_{L_{1}}^{(e r)}\right)_{L_{1}, \sigma_{1}}=0
$$

where $a_{L_{1}} \in F\left(L_{1}\right)$ is the pullback of $a$.

Proof. We have $a_{L_{1}} \in \tilde{F}\left(\mathcal{O}_{L_{1}}, \mathfrak{m}_{L_{1}}^{-e r}\right)$ and hence the statement follows from the construction of the symbol in Section 4.37 and (LS3). 
Lemma 4.41. Set $F \in \mathbf{R S C}_{\mathrm{Nis}}$. Let $K / k$ be a function field and $X$ be a normal affine integral finite-type $K$-scheme with function field $E$. Let $x_{i} \in X^{(1)}, i=1, \ldots, r$, be distinct 1-codimensional points. Then for all integers $n_{i} \geq 0$, the natural map

$$
\frac{F(E)}{\cap_{i=1}^{r} \tilde{F}\left(\mathcal{O}_{X, x_{i}}, \mathfrak{m}_{x_{i}}^{-n_{i}}\right)} \stackrel{\simeq}{\longrightarrow} \prod_{i=1}^{r} \frac{F(E)}{\tilde{F}\left(\mathcal{O}_{X, x_{i}}, \mathfrak{m}_{x_{i}}^{-n_{i}}\right)}
$$

is an isomorphism, where $\tilde{F}\left(\mathcal{O}_{X, x_{i}}, \mathfrak{m}_{x_{i}}^{0}\right):=F\left(\mathcal{O}_{X, x_{i}}\right)$.

Proof. Let $A$ be the semilocalisation of $X$ at the points $x_{i}$ and denote by $D=\sum_{i} n_{i} x_{i}$ the divisor on $U:=\operatorname{Spec} A$. (Note that we allow $|D| \varsubsetneqq\left\{x_{1}, \ldots, x_{r}\right\}$.) We claim

$$
\tilde{F}(U, D)=\cap_{i=1}^{r} \tilde{F}\left(\mathcal{O}_{X, x_{i}}, \mathfrak{m}_{x_{i}}^{-n_{i}}\right) .
$$

Indeed, by definition, $\tilde{F}(U, D)=\tilde{F}_{(U, D)}(U)$; furthermore, $\tilde{F}_{(U, D)}$ is a sheaf on $U_{\text {Nis }}$ and is a subsheaf of the constant sheaf $F(K)$ (by [26, Theorem 6] and [27, Corollary 3.2.3]). Since $\operatorname{Spec} E \sqcup_{i} \operatorname{Spec} \mathcal{O}_{X, x_{i}} \rightarrow U$ is a Nisnevich cover, the claim follows.

The natural map in the statement is compatible with pullbacks and push-forwards on both sides. Thus we can apply the standard trick replacing $k$ by its maximal pro$\ell$ extensions for various primes $\ell$, to assume $k$ is infinite. By Gabber's presentation theorem (see, for example, [8, 3.1.2]), we find a function field $K_{1} / k$ and an essentially étale morphism $\varphi: U \rightarrow \mathbf{A}_{K_{1}}^{1}$ such that $\left\{x_{1}, \ldots, x_{r}\right\}=\varphi^{-1} \varphi\left(\left\{x_{1}, \ldots, x_{r}\right\}\right) \cong \varphi\left(\left\{x_{1}, \ldots, x_{r}\right\}\right)$. By [45, Lemma 4.2, Lemma 4.3], $\left(U, \sum_{i} m_{i} x_{i}\right)$ is a $V$-pair for all $m_{i} \geq 0$. Let $U^{h}$ be the henselisation of $U$ with respect to the radical in $A$ (see [42, XI, §2, Théorème 2]) and set $D^{h}:=D_{\mid U^{h}}$; by $[45$, Lemma $4.4,(2),(3)]$, we have an isomorphism

$$
F\left(U \backslash\left\{x_{1}, \ldots, x_{r}\right\}\right) / \tilde{F}(U, D) \stackrel{\simeq}{\longrightarrow} F\left(U^{h} \backslash\left\{x_{1}, \ldots, x_{r}\right\}\right) / \tilde{F}\left(U^{h}, D^{h}\right) .
$$

Now the statement follows from $U^{h}=\sqcup_{i} \operatorname{Spec} \mathcal{O}_{X, x_{i}}^{h}$ (see [42, XI, §2, Proposition 1, 1)], equation (4.41.1) and Lemma 4.36).

Lemma 4.42. Set $F \in \mathbf{R S C}_{\mathrm{Nis}}$ and let $\pi: \operatorname{Spec} L^{\prime} \rightarrow \operatorname{Spec} L$ be a finite extension of henselian dvfs. Denote by $\sigma: K \rightarrow \mathcal{O}_{L}$ a $k$-morphism such that the composition $L \rightarrow$ $\mathcal{O}_{L} / \mathfrak{m}_{L}$ is a finite field extension; denote by $\sigma^{\prime}: K \rightarrow \mathcal{O}_{L^{\prime}}$ the induced map. Then we have

(1) $\left(\pi_{*} b, f\right)_{L, \sigma}=\left(b, \pi^{*} f\right)_{L^{\prime}, \sigma^{\prime}}, \quad b \in F\left(L^{\prime}\right), f \in \mathcal{O}_{L}^{\times}$;

(2) $\left(a, \pi_{*} g\right)_{L, \sigma}=\left(\pi^{*} a, g\right)_{L^{\prime}, \sigma^{\prime}}, \quad a \in F(L), g \in \mathcal{O}_{L^{\prime}}^{\times}$

Proof. We can spread out the situation as follows: There exists a finite and surjective morphism $\bar{\pi}: C^{\prime} \rightarrow C$ between regular and projective $K$-curves, with function fields $E^{\prime}=$ $K\left(C^{\prime}\right), E=K(C)$, points $x^{\prime} \in C^{\prime}$ and $x=\bar{\pi}\left(x^{\prime}\right) \in C$ and elements $\tilde{a} \in F(E), \tilde{b} \in F\left(E^{\prime}\right)$, $\tilde{f} \in \mathcal{O}_{C, x}^{\times}, g \in \mathcal{O}_{C^{\prime}, x^{\prime}}^{\times}$inducing $\pi, \sigma, \sigma^{\prime}, a, b, f, g$. We prove (1): by Lemma 4.41 we find an element $b_{1} \in F\left(E^{\prime}\right)$ with $\tilde{b}-b_{1} \in F\left(\mathcal{O}_{C^{\prime}, x^{\prime}}\right)$, and $b_{1} \in F\left(\mathcal{O}_{C^{\prime}, y}\right)$ for all $y \in \bar{\pi}^{-1}(x) \backslash\left\{x^{\prime}\right\}$. Since $\bar{\pi}^{*} \tilde{f} \in \mathcal{O}_{C^{\prime}, y}^{\times}$, for all $y / x$, we obtain

$$
\left(b, \pi^{*} f\right)_{L^{\prime}, \sigma^{\prime}} \stackrel{(\mathrm{LS} 1),(\mathrm{LS} 2)}{=} \sum_{y / x}\left(b_{1}, \bar{\pi}^{*} f\right)_{C^{\prime} / K, y} \stackrel{(\mathrm{LS} 7)}{=}\left(\bar{\pi}_{*}\left(b_{1}\right), \tilde{f}\right)_{C / K, x} .
$$


Note that $E^{\prime} \otimes_{E} L \cong \prod_{y / x} E_{y}^{\prime}$, where $E_{y}^{\prime}$ is the henselisation of $E^{\prime}$ at $y$. Thus in $F(L)$ we have

$$
\bar{\pi}_{*} b_{1}=\sum_{y / x} \pi_{y *} b_{1},
$$

where $\pi_{y}: \operatorname{Spec} L \rightarrow \operatorname{Spec} E_{y}^{\prime}$ is the natural map; in particular, $\pi_{x^{\prime}}=\pi$. Hence in $F(L)$,

$$
\bar{\pi}_{*} b_{1} \equiv \pi_{*} b \bmod F\left(\mathcal{O}_{L}\right) ;
$$

this together with equation (4.42.1) and (LS1) implies (1).

Now for (2): by the approximation lemma we find $g_{1} \in E^{\prime \times}$ such that

$$
\left(\bar{\pi}^{*} \tilde{a}, g_{1}\right)_{C^{\prime} / K, y}=0, \quad y \in \pi^{-1}(x) \backslash\left\{x^{\prime}\right\},
$$

and

$$
\left(\bar{\pi}^{*} \tilde{a}, g_{1}\right)_{C^{\prime} / K, x^{\prime}}=\left(\pi^{*} a, g\right)_{L^{\prime}, \sigma^{\prime}} .
$$

Furthermore, we have the following equality in $L^{\times}$:

$$
\mathrm{Nm}_{E^{\prime} / E}\left(g_{1}\right)=\prod_{y / x} \mathrm{Nm}_{E_{y}^{\prime} / L}\left(g_{1}\right) \text {. }
$$

If $g_{1}$ is close enough to 1 at the points $y \in \bar{\pi}^{-1}(x) \backslash\left\{x^{\prime}\right\}$, we have $\mathrm{Nm}_{E_{y}^{\prime} / L}\left(g_{1}\right) \in U_{L}^{(N)}$ for $N \gg 0$. Thus we can choose $g_{1}$ with the additional property

$$
\left(\tilde{a}, \mathrm{Nm}_{E^{\prime} / E}\left(g_{1}\right)\right)_{C / K, x}=\left(a, \mathrm{Nm}_{L^{\prime} / L}(g)\right)_{L, \sigma} .
$$

(2) now follows from (LS8) and the foregoing.

\section{Part 2. Applications}

\section{Algebraic groups and the local symbol}

In this section, $k$ is a perfect field and $G$ is a commutative algebraic $k$-group. Note that as sheaves on Sm, we have $G=G_{\text {red }}$, and hence we can always identify $G$ with the smooth commutative $k$-group $G_{\text {red }}$. We fix an algebraic closure $\bar{k}$ of $k$; note that $\operatorname{Spec} \bar{k} \in \mathbf{C o r}^{\text {pro }}$.

5.1. Let $G$ be a commutative algebraic $k$-group. Then $G \in \mathbf{R S C}_{\mathrm{Nis}}$, by [27, Corollary 3.2.5]. Let $L \in \Phi_{\leq 1}$ have residue field $\kappa$. Let $\iota: \kappa \hookrightarrow \bar{k}$ be a $k$-embedding. We denote by $L_{\iota}^{s h}$ the strict henselisation of $L$ with respect to $\iota$. Note that $L_{\iota}^{s h}$ is a henselian dvf of geometric type over $\bar{k}$. We write

$$
(-,-)_{L_{\iota}^{s h}}: G\left(L_{\iota}^{s h}\right) \times L_{\iota}^{s h \times} \rightarrow G(\bar{k})
$$

for the symbol $(-,-)_{L_{\iota}^{s h}, \sigma}$ from Section 4.37, with $\sigma: \bar{k} \hookrightarrow \mathcal{O}_{L, \iota}^{s h}$ the unique coefficient field; in this case this $i s$ the symbol defined by Rosenlicht and Serre (see [47, III, §1]). If we choose a different $k$-embedding $\iota^{\prime}: \kappa \hookrightarrow \bar{k}$, then we find an automorphism $\tau: \bar{k} \rightarrow \bar{k}$ 
with $\tau \circ \iota=\iota^{\prime}$ inducing a unique isomorphism of $\mathcal{O}_{L^{-}}$-algebras $\tau: \mathcal{O}_{L, \iota}^{s h} \stackrel{\simeq}{\rightarrow} \mathcal{O}_{L, \iota^{\prime}}^{h}$ and by equation (4.38.1),

$$
\tau\left((a, f)_{L_{\iota}^{s h}}\right)=(\tau(a), \tau(f))_{L_{\iota^{\prime}}^{s h}} .
$$

We will usually drop the $\iota$ from the notation and write $L^{s h}=L_{\iota}^{s h}$. We define the Rosenlicht-Serre conductor of $a \in G(L)$ by

$$
\operatorname{RoSe}_{L}(a):= \begin{cases}0 & \text { if } a \in G\left(\mathcal{O}_{L}\right) \\ \min \left\{n \geq 1 \mid\left(a, U_{L^{s h}}^{(n)}\right)_{L^{s h}}=0\right\} & \text { otherwise }\end{cases}
$$

Note that it is independent of the choice of $\iota: \kappa \hookrightarrow \bar{k}$.

Theorem 5.2. Let $G$ be a commutative algebraic $k$-group.

(1) The Rosenlicht-Serre conductor $\operatorname{RoSe}=\left\{\operatorname{RoSe}_{L}\right\}_{\operatorname{trdeg}(L / k)=1}$ is a semicontinuous conductor of level 1 on $G$ (in the sense of Definitions 4.3 and 4.22).

(2) Let $c^{G}$ be the motivic conductor of $G$ (see Definition 4.14) and denote by $\left(c^{G}\right)^{\leq 1}$ its restriction to $\Phi_{\leq 1}$. Then $\operatorname{RoSe}=\left(c^{G}\right)^{\leq 1}$.

In particular, the motivic conductor extends the Rosenlicht-Serre conductor to henselian $d v f$ s over $k$ with nonperfect residue field, and we have $\widetilde{G}=\widetilde{G}_{\mathrm{RoSe}}$ (see Section 4.8 and Notation 4.17 for notation).

Proof. The last statement follows from Corollary 4.18. For (1) we check that RoSe satisfies the properties from Definition 4.3. (c1) and (c2) are obvious. Let $L^{\prime} / L$ be a finite extension of henselian dvfs with $\operatorname{trdeg}(L / k)=1$ and $a \in G\left(L^{\prime}\right)$. Let $\kappa \hookrightarrow \kappa^{\prime}$ be the induced map on the residue fields, and fix an embedding $\kappa^{\prime} \subset \bar{k}$. Then $L^{\prime s h}$ is finite over $L^{s h}$ and $e\left(L^{\prime s h} / L^{s h}\right)=e\left(L^{\prime} / L\right)$. Thus (c3) follows directly from Lemma 4.42(1). To check (c4), first observe that if $a \in G\left(\mathbf{A}_{X}^{1}\right)$ is not in $G(X)$ (via pullback), then we find a closed point $x \in X$ such that $a_{\mathbf{A}_{x}^{1}}$ is not in $G(x)$. (Since $G$ is a finite-type $k$-scheme and $X$ is Jacobson.) Thus it suffices to show the following:

Claim. Let $\kappa / k$ be a finite field extension and set $\kappa(t)_{\infty}=\operatorname{Frac}\left(\mathcal{O}_{\mathbf{P}_{\kappa}^{1}, \infty}^{h}\right)$. Assume that $a \in G\left(\mathbf{A}_{\kappa}^{1}\right)$ has $\operatorname{RoSe}_{\kappa(t)_{\infty}}(a) \leq 1$. Then $a \in G(\kappa)$.

Otherwise $a \notin G(\kappa)$. Then its pullback $a_{\bar{k}} \in G\left(\mathbf{A}_{\bar{k}}^{1}\right)$ is not in $G(\bar{k})$, and we can thus find two points $x, y \in \mathbf{A}^{1}(\bar{k})=\bar{k}$ such that $a_{\bar{k}}(x) \neq a_{\bar{k}}(y)$. Take $f=(t-x) /(t-y) \in \bar{k}(t)$. Then $f \in U_{\bar{k}(t)_{\infty}}^{(1)}$, and we obtain

$$
0=\left(a_{\bar{k}}, f\right)_{\bar{\kappa}(t)_{\infty}}=-a_{\bar{k}}(x)+a_{\bar{k}}(y),
$$

where the first equality follows from $\operatorname{RoSe}_{\kappa(t)_{\infty}}(a) \leq 1$ and the second from (LS4) and (LS2). This yields a contradiction and thereby proves the claim. (c5) follows from the fact that $G$ is a reciprocity sheaf and Corollary 4.40. Finally, we deal with (c6) (continuity for $n=1)$. Assume that $C$ is a smooth $k$-curve, $x \in C$ is a closed point and $a \in G(C \backslash\{x\})$ 
with $\operatorname{RoSe}_{L_{x}}\left(a_{x}\right) \leq n$, where $L_{x}=\operatorname{Frac}\left(\mathcal{O}_{C, x}^{h}\right)$ and $a_{x} \in G\left(L_{x}\right)$ denotes the pullback of $a$. Let $\bar{C}$ be the smooth compactification of $C$ and let $C_{\infty}=(\bar{C} \backslash C)_{\text {red }}$. Choose $N$ such that $\operatorname{RoSe}_{L_{y}}\left(a_{y}\right) \leq N$ for all $y \in\left|C_{\infty}\right|$. Then $\left(\bar{C}, n \cdot\{x\}+N \cdot C_{\infty}\right)$ is a compactification of $(C, n \cdot\{x\})$ and we claim

$$
\operatorname{RoSe}_{\bar{C}}(a) \leq\left(n \cdot\{x\}+N \cdot C_{\infty}\right) .
$$

Indeed, let $\operatorname{Spec} L \rightarrow C \backslash\{x\}$ be a henselian dvf point with $\operatorname{trdeg}(L / k)=1$. If $\operatorname{Spec} \mathcal{O}_{L}$ maps to $C \backslash\{x\}$, then $\operatorname{RoSe}_{L}\left(a_{L}\right)=0$. Otherwise we get a finite extension $L^{s h} / L_{y}^{s h}$ for some $y \in\{x\} \cup\left|C_{\infty}\right|$, say of ramification index $e$. Set $u \in U_{L^{s h}}^{\left(n_{y} e\right)}$, where $n_{x}=n$ and $n_{y}=N$, for $y \neq x$. By Lemma 4.42(2) we have

$$
\left(a_{L}, u\right)_{L^{s h}}=\left(a_{L_{y}}, \operatorname{Nm}_{L^{s h} / L_{y}^{s h}}(u)\right)_{L_{y}^{s h}},
$$

which vanishes by $\operatorname{Nm}_{L^{s h} / L_{y}^{s h}}(u) \in U_{L_{y}^{s h}}^{\left(n_{y}\right)}$ and $\operatorname{RoSe}_{L_{y}}\left(a_{y}\right) \leq n_{y}$. This proves claim (5.2.1), hence (c6), and finishes the proof of (1).

By Corollary 4.24, we have $c^{G, 1} \leq$ RoSe. Thus for (2) it suffices to show that if $a \in \tilde{G}\left(\mathcal{O}_{L}, \mathfrak{m}_{L}^{-r}\right)$, for some $L \in \Phi_{\leq 1}$ and $r \geq 1$, then $\operatorname{RoSe}_{L}(a) \leq r$. This follows from Corollary 4.40.

Remark 5.3. An extension of RoSe to dvfs of higher transcendence degree over $k$ was also constructed in [33] (char 0) and [32] (char $p>0$ ). The construction essentially coincides with the extension from Theorem 5.2, but considering the log version, whereas here the nonlog one is considered (compare Theorem 7.20).

\section{Differential forms and irregularity of rank 1 connections}

In this section we assume that the base field $k$ has characteristic 0 . We fix a ring homomorphism $R \rightarrow k$ which induces the structure of an $R$-scheme on any $k$-scheme. (Of main interest are $R=k$ or $\mathbb{Z}$.)

\subsection{Kähler differentials}

6.1. Set $X \in \mathbf{S m}$. We denote by $\Omega_{X / R}^{\bullet}$ the de Rham complex on $X$ relative to $R$, and by $d: \Omega_{X / R}^{\bullet} \rightarrow \Omega_{X / R}^{\bullet+1}$ the differential. We set $\Omega_{X}^{\bullet}:=\Omega_{X / \mathbb{Z}}^{\bullet}$. We have an exact sequence

$$
\Omega_{R}^{1} \otimes_{R} \Omega_{X}^{\bullet-1} \rightarrow \Omega_{X}^{\bullet} \rightarrow \Omega_{X / R}^{\bullet} \rightarrow 0 .
$$

We denote the Nisnevich sheaf on Sm given by $X \mapsto H^{0}\left(X, \Omega_{X / R}^{q}\right)$ by $\Omega_{/ R}^{q}$ and set $\Omega^{q}:=\Omega_{/ \mathbb{Z}}^{q}$. By [26, Theorem A.6.2] and [27, Corollary 3.2.5], we have $\Omega^{q} \in \mathbf{R S C}_{\mathrm{Nis}}$. Since the action of finite correspondences on $\Omega^{\bullet}$ is $\Omega_{k^{\bullet}}$-linear (a fortiori, it is $\Omega_{R^{\bullet}}$-linear), the morphism $\alpha^{*}: \Omega^{q}(Y) \rightarrow \Omega^{q}(X), \alpha \in \operatorname{Cor}(X, Y)$, induces via sequence (6.1.1) the structure of a Nisnevich sheaf with transfers on $\Omega_{/ R}^{q}$, and we obtain $\Omega_{/ R}^{q} \in \mathbf{R S C}_{\mathrm{Nis}}$.

Lemma 6.2. The differential $d: \Omega_{/ R}^{q} \rightarrow \Omega_{/ R}^{q+1}$ is a map in $\mathbf{R S C}_{\mathrm{Nis}}$. 
Proof. We have to show that if $\alpha \in \mathbf{C o r}(X, Y)$ is a finite correspondence, $X, Y \in \mathbf{S m}$, then $\alpha^{*} d=d \alpha^{*}$ as maps $\Omega_{/ R}^{q}(Y) \rightarrow \Omega_{/ R}^{q}(X)$. Since the restriction $\Omega_{/ R}^{q}(X) \rightarrow \Omega_{/ R}^{q}(U)$ is injective for any dense open $U \subset X$ (by [26, Theorem 6]), it suffices to verify the equality after shrinking $X$ arbitrarily around its generic points. In particular, we can assume that $X$ is connected and $\alpha=Z \subset X \times Y$ is a prime correspondence which is finite étale over $X$ (here we use $\operatorname{char}(k)=0$ ). Denote by $f: Z \rightarrow X$ and $g: Z \rightarrow Y$ the maps induced by projection. Then $Z^{*}=f_{*} g^{*}$. The compatibility of $d$ with $g^{*}$ is clear. Hence it remains to show $f_{*} d=d f_{*}$ for a finite étale map $f: Z \rightarrow X$ between smooth schemes. In this case, we have $f_{*} \Omega_{Z / R}^{q}=f_{*} \mathcal{O}_{Z} \otimes_{\mathcal{O}_{X}} \Omega_{X / R}^{q}$ and $f_{*}=\operatorname{Tr}_{f} \otimes \operatorname{id}_{\Omega_{/ R}^{q}}$, by [7, Proposition 2.2.23]. Thus the compatibility we seek is shown as in [20, II, Proof of Proposition (2.2), case 2].

6.3. Set $L \in \Phi$, with local parameter $t \in \mathfrak{m}_{L} \subset \mathcal{O}_{L}$. We denote by $\Omega_{\mathcal{O}_{L} / R}(\log )$ the differential graded algebra of logarithmic differentials - that is, the graded subalgebra of $\Omega_{L / R}^{\bullet}$ generated by $\Omega_{\mathcal{O}_{L} / R}^{\bullet}$ and dlog $t$. In particular, $\Omega_{\mathcal{O}_{L} / R}^{0}(\log )=\mathcal{O}_{L}$. For $q \geq 0$ and $a \in \Omega_{L / R}^{q}$, we define

$$
c_{L}^{\mathrm{dR}}(a):= \begin{cases}0 & \text { if } a \in \Omega_{\mathcal{O}_{L} / R}^{q}, \\ \min \left\{n \geq 1 \mid a \in \frac{1}{t^{n-1}} \cdot \Omega_{\mathcal{O}_{L} / R}^{q}(\log )\right\} & \text { otherwise. }\end{cases}
$$

Theorem 6.4. For all $q \geq 0$, the collection $c^{\mathrm{dR}}=\left\{c_{L}^{\mathrm{dR}}\right\}$ defined in Section 6.3 coincides with the motivic conductor - that is (see Definition 4.14),

$$
c^{\mathrm{dR}}=c^{\Omega_{/ R}^{q}}
$$

Furthermore, the restriction $\left(c^{\mathrm{dR}}\right)^{\leq q+1}$ is a semicontinuous conductor.

Proof. We start by showing that $c^{\mathrm{dR}}$ is a semicontinuous conductor of level $q+1$. Properties (c1) and (c2) from Definition 4.3 are obvious.

(c3). Let $L^{\prime} / L$ be a finite extension of a henselian dvf with ramification index $e=$ $e\left(L^{\prime} / L\right)$, and denote by $f: \operatorname{Spec} L^{\prime} \rightarrow \operatorname{Spec} L$ the induced map. Set $a \in \Omega_{L^{\prime} / R}^{q}$. We have to show

$$
c_{L}^{\mathrm{dR}}\left(f_{*} a\right) \leq\left\lceil\frac{c_{L^{\prime}}^{\mathrm{dR}}(a)}{e}\right\rceil .
$$

We know that $f_{*}$ restricts to $\Omega_{\mathcal{O}_{L^{\prime}} / R}^{q} \rightarrow \Omega_{\mathcal{O}_{L} / R}^{q}$, and by the well-known formula $f_{*} \operatorname{dlog}=$ $\operatorname{dlog} \circ \mathrm{Nm}_{L^{\prime} / L}$ also to

$$
f_{*}: \Omega_{\mathcal{O}_{L^{\prime}} / R}^{q}(\log ) \rightarrow \Omega_{\mathcal{O}_{L} / R}^{q}(\log )
$$

Moreover, $f_{*}$ is continuous with respect to the $\mathfrak{m}_{L^{-}}$-adic topology (which on $\Omega_{L^{\prime} / R}^{q}$ is

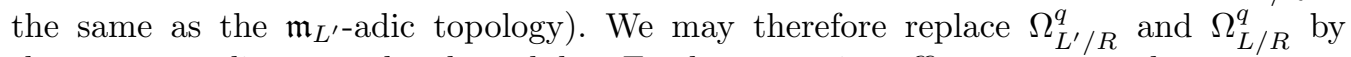
the corresponding completed modules. Furthermore, it suffices to treat the two cases separately in which $L^{\prime} / L$ is either totally ramified or unramified.

Case 1: $e=1$. In this case, a local parameter $t \in \mathcal{O}_{L}$ is also a local parameter of $\mathcal{O}_{L^{\prime}}$ and hence formula (6.4.1) follows directly from expression (6.4.2) and the $L$-linearity of $f_{*}$. 
Case 2: $e>1, L, L^{\prime}$ complete and $\mathcal{O}_{L} / \mathfrak{m}_{L}=\mathcal{O}_{L^{\prime}} / \mathfrak{m}_{L^{\prime}}$. Let $K \hookrightarrow \mathcal{O}_{L}$ be a coefficient field; it also defines a coefficient field of $\mathcal{O}_{L^{\prime}}$. Let $\tau \in \mathcal{O}_{L^{\prime}}$ and $t \in \mathcal{O}_{L}$ be local parameters. Then we can identify $L^{\prime}=K((\tau))$ and $\frac{1}{\tau^{n-1}} \cdot \widehat{\Omega}_{\mathcal{O}_{L^{\prime}} / R}^{q}(\log )$ with the $\tau$-adic completion of

$$
\frac{1}{\tau^{n-1}} \cdot\left(\left(K[\tau] \otimes_{K} \Omega_{K / R}^{q}\right) \oplus\left(K[\tau] \operatorname{dlog} \tau \otimes_{K} \Omega_{K / R}^{q-1}\right)\right) .
$$

Furthermore, observe that $\frac{1}{\tau^{i}} \operatorname{d} \log \tau=-\frac{1}{i} d\left(\frac{1}{\tau^{i}}\right), i \geq 1$. Since $f_{*}$ commutes with the differential (by Lemma 6.2), we are reduced to showing

$$
f_{*}\left(\frac{1}{\tau^{i}}\right) \in \frac{1}{t^{r-1}} \mathcal{O}_{L}, \quad r:=\left\lceil\frac{n}{e}\right\rceil, \text { for all } i \in[1, n-1] .
$$

We compute for $i \in[1, n-1]$

$$
\begin{aligned}
\mathfrak{m}_{L}^{r} \cdot d f_{*}\left(\frac{1}{\tau^{i}}\right) & =\mathfrak{m}_{L}^{r} \cdot f_{*}\left(-i \tau^{-i-1} d \tau\right) \\
& \subset f_{*}\left(\mathfrak{m}_{L^{\prime}}^{r-i-1} d \tau\right) \\
& \subset f_{*}\left(\Omega_{\mathcal{O}_{L^{\prime}} / R}^{1}\right) \subset \Omega_{\mathcal{O}_{L} / R}^{1}
\end{aligned}
$$

This implies formula (6.4.3), once we observe that in characteristic 0 we have $\mathfrak{m}_{L}^{r} \cdot d a \in$ $\Omega_{\mathcal{O}_{L} / R}^{1}$ if and only if $\mathfrak{m}_{L}^{r-1} \cdot a \in \mathcal{O}_{L}$, for any $a \in L=K((t))$.

(c4). For $c^{\mathrm{dR}, q+1}$, it follows directly from the following facts, where $A$ is a finite-type smooth $k$-algebra:

(i) $\Omega_{A[t] / R}^{q}=\left(k[t] \otimes_{k} \Omega_{A / R}^{q}\right) \oplus\left(\Omega_{A / R}^{q-1} \otimes_{k} \Omega_{k[t] / k}^{1}\right)$;

(ii) for any nonzero $\alpha \in \Omega_{A / R}^{q}$, there exists a prime ideal $\mathfrak{p} \subset A$ with $\operatorname{trdeg}(k(\mathfrak{p}) / k)=q$, where $k(\mathfrak{p})=A_{\mathfrak{p}} / \mathfrak{p}$, such that the image of $\alpha$ in $\Omega_{k(\mathfrak{p}) / R}^{q}$ is nonzero;

(iii) $H^{0}\left(\mathbf{P}_{k}^{1}, \Omega_{\mathbf{P}^{1} / k}^{1}(\log \infty)\right)=0, H^{0}\left(\mathbf{P}^{1}, \mathcal{O}_{\mathbf{P}^{1}}\right)=k$.

(Note that 6.1 is easy for $R=k$ and follows in general from the natural map $\Omega_{/ R}^{q} \rightarrow \Omega_{/ k}^{q}$.)

For (c5) it suffices to observe that if $a \in H^{0}\left(X, \Omega_{X / R}^{q} \otimes_{\mathcal{O}_{X}} \mathcal{O}_{X}(D)\right)$ for some proper modulus pair $(X, D)$, then $c_{X}^{\mathrm{dR}}(a) \leq D$.

Finally, (c6). Let $U=\operatorname{Spec} A$ be smooth affine and $Z \subset U$ be a smooth divisor which we can assume to be principal $Z=\operatorname{Div}(t)$. Let

$$
a=\frac{1}{t^{r-1}} a_{1}+\frac{1}{t^{r-1}} a_{2} \operatorname{dlog} t, \quad a_{1} \in \Omega_{A / R}^{q}, a_{2} \in \Omega_{A / R}^{q-1}, r \geq 1 .
$$

Let $(\bar{Y}, \bar{Z}+\Sigma)$ be a compactification of $(U, Z)$ with $\bar{Z}_{\mid U}=Z$ and $\bar{Y}$ normal. Let $\bar{Y}=\cup V_{i}$ be an open covering such that $V_{i}=\operatorname{Spec} B_{i}, \Sigma_{\mid V_{i}}=\operatorname{Div}\left(f_{i}\right)$ and $\bar{Z}_{\mid V_{i}}=\operatorname{Div}\left(\tau_{i}\right)$, with $\tau_{i}, f_{i} \in B_{i}$. Note that $\operatorname{Spec} B_{i}\left[1 / f_{i}\right] \subset U$ is open for all $i$. Hence, in $B_{i}\left[1 / f_{i}\right]$ we can write $t=\tau_{i} e_{i}$, with $e_{i} \in\left(B_{i}\left[1 / f_{i}\right]\right)^{\times}$. Let $E_{i}$ be the Cartier divisor on $V_{i}$ defined by $e_{i}$. We have $\left|E_{i}\right| \subset\left|\Sigma_{\mid V_{i}}\right|$. By Lemma 6.5, there exists $N_{1} \gg 0$ such that $v_{L}\left(E_{i}\right) \leq N_{1} v_{L}\left(\Sigma_{\mid V_{i}}\right)$ for all Spec $L \rightarrow U$ and all $i$. Furthermore, there exists an $N_{2} \geq 0$ such that $f_{i}^{N_{2}} a_{1} \in \Omega_{B_{i} / R}^{q}$ and $f_{i}^{N_{2}} a_{2} \in \Omega_{B_{i} / R}^{q-1}$ for all $i$. Choose $N \geq r \cdot N_{1}+N_{2}$. Set $\rho: \operatorname{Spec} L \rightarrow U, L \in \Phi$. Assume that 
the closed point of $\operatorname{Spec} \mathcal{O}_{L}$ maps into $|\bar{Z}+\Sigma| \cap V_{i}$ for some $i$. Then

$$
\begin{aligned}
c_{L}\left(\rho^{*} a\right) & \leq(r-1) v_{L}(\bar{Z})+(r-1) v_{L}\left(E_{i}\right)+N_{2} v_{L}(\Sigma)+1 \\
& \leq(r-1) v_{L}(\bar{Z})+(r-1) N_{1} v_{L}(\Sigma)+N_{2} v_{L}(\Sigma)+1 \\
& \leq v_{L}(r \cdot \bar{Z}+N \cdot \Sigma) .
\end{aligned}
$$

Hence $c_{\bar{Y}}^{\mathrm{dR}}(a) \leq(r \cdot \bar{Z}+N \cdot \Sigma)$, which proves $(\mathrm{c} 6)$.

Thus $c^{\mathrm{dR}}$ is a semicontinuous conductor on $\Omega_{/ R}^{q}$ and Theorem 4.15(3) yields, for $n \geq 1$,

$$
\mathrm{fil}_{n}:=\frac{1}{t^{n-1}} \cdot \Omega_{\mathcal{O}_{L} / R}^{q}(\log ) \subset \widetilde{\Omega_{/ R}^{q}}\left(\mathcal{O}_{L}, \mathfrak{m}_{L}^{-n}\right)
$$

for any $L \in \Phi$ with local parameter $t \in \mathcal{O}_{L}$. It remains to show the other inclusion. By Corollary 4.40 it suffices to show the following: let $K \hookrightarrow \mathcal{O}_{L}$ be some coefficient field and extend it in the canonical way to $\sigma: K(x) \hookrightarrow \mathcal{O}_{L_{x}}$, where $x$ is a variable and $L_{x}=\operatorname{Frac}\left(\mathcal{O}_{L}[x]_{(t)}^{h}\right)$. Assume $a \in$ fil $_{r+1}$. Then the following implication holds:

$$
\left(a, 1-x t^{r}\right)_{L_{x}, \sigma}=0 \Rightarrow a \in \mathrm{fil}_{r},
$$

where the local symbol on the left-hand side is the one from Section 4.37 for $\Omega_{/ R}^{q}$. Since the local symbol for $\Omega_{/ R}^{q}$ is uniquely determined by (LS1)-(LS4), we see that it is given by

$$
\left(a, 1-x t^{r}\right)_{L_{x}, \sigma}=\operatorname{Res}_{t}\left(a \operatorname{dlog}\left(1-x t^{r}\right)\right)
$$

where we use the isomorphism $L_{x}=K(x)((t))$ defined by $\sigma$ to compute the residue symbol on the right. To prove implication (6.4.4), it suffices to consider $a$ modulo fil $r$; we have

$$
a \equiv \frac{1}{t^{r}} \alpha+\beta \frac{d t}{t^{r+1}} \bmod \mathrm{fil}_{r}
$$

for $\alpha \in \Omega_{K / R}^{q}, \beta \in \Omega_{K / R}^{q-1}$. We compute in $\Omega_{K(x) / R}^{q}$

$$
\operatorname{Res}_{t}\left(a \operatorname{dlog}\left(1-x t^{r}\right)\right)=-r x \alpha+\beta d x .
$$

This shows implication (6.4.4) and completes the proof.

Lemma 6.5. Let $X$ be a Noetherian integral normal scheme and $E$ and $F$ be two Cartier divisors on $X$; assume that $F$ is effective. If $|E| \subset|F|$, then there exists $N \geq 1$ such that for all maps $\operatorname{Spec} \mathcal{O} \rightarrow X$ whose image is not contained in $|F|$, with $\mathcal{O}$ a DVR with valuation $v$, we have $v(E) \leq N \cdot v(F)$.

Proof. The question is local on $X$; hence we can assume $E$ and $F$ are given by functions $e, f \in k(X)^{\times}$. Let $\operatorname{Div}(e)$ and $\operatorname{Div}(f)$ be the associated Weil divisors. Since $|E| \subset|F|$ and $F$ is effective, we find $N \geq 1$ such that $\operatorname{Div}(e) \leq N \cdot \operatorname{Div}(f)$, which by the normality of $X$ implies $f^{N} / e \in \Gamma\left(X, \mathcal{O}_{X}\right)$. This yields the statement. 
Remark 6.6. The proof of Theorem 6.4 also shows that

$$
c_{L}^{\mathrm{dR}^{\prime}}(a)= \begin{cases}0 & \text { if } a \in \Omega_{\mathcal{O}_{L} / R}^{q}, \\ \min \left\{n \geq 2 \mid a \in \frac{1}{t^{n-1}} \cdot \Omega_{\mathcal{O}_{L} / R}^{q}\right\} & \text { otherwise }\end{cases}
$$

defines a semicontinuous conductor on $\Omega^{q}$, but it coincides with the motivic one only for $q=0$.

Corollary 6.7. Set $Z \Omega_{/ R}^{q}=\operatorname{Ker}\left(d: \Omega_{/ R}^{q} \rightarrow \Omega_{/ R}^{q+1}\right)$. Then $Z \Omega_{/ R}^{q} \in \mathbf{R S C}_{\mathrm{Nis}}$, and its motivic conductor $c^{Z \Omega_{/ R}^{q}}=\left(c^{\Omega_{/ R}^{q}}\right)_{\mid Z \Omega_{/ R}^{q}}$ restricts to a conductor of level $q$.

Proof. The formula for $c^{Z \Omega_{/ R}^{q}}$ follows from Proposition 4.19. It remains to show that it has level $q$. Set $a \in Z \Omega_{/ R}^{q}\left(\mathbf{A}_{X}^{1}\right)$ with $c_{k(x)(t)_{\infty}}^{\mathrm{dR}}(a) \leq 1$, for all points $x \in X$ with $\operatorname{trdeg}(k(x) / k) \leq$ $q-1$. This implies $a \in H^{0}\left(X, k[t] \otimes_{k} \Omega_{X / R}^{q}\right) \cap Z \Omega_{/ R}^{q}\left(\mathbf{A}_{X}^{1}\right)$ (compare the proof of (c4) in Theorem 6.4). Hence $a \in Z \Omega_{/ R}^{q}(X)$. This shows that $\left(c^{Z \Omega_{/ R}^{q}}\right)^{\leq q}$ satisfies (c4).

\section{Corollary 6.8.}

(1) Let $\mathcal{X}=(X, D) \in$ MCor be a proper modulus pair. Then

$$
\widetilde{\Omega_{/ R}^{q}}(\mathcal{X})=H^{0}\left(X_{1}, \Omega_{X_{1} / R}^{q}\left(\log D_{1}\right)\left(D_{1}-D_{1, \text { red }}\right)\right)
$$

where $\pi: X_{1} \rightarrow X$ is any resolution of singularities which is an isomorphism over $X \backslash D$ and such that $D_{1}:=\pi^{*} D$ is supported on a simple normal crossings divisor. (See Notation 4.17 for the notation $\widetilde{\Omega_{/ R}^{q}}$.)

(2) Let $h_{\mathbf{A}^{1}}^{0}\left(\Omega_{/ R}^{q}\right)$ be the maximal $\mathbf{A}^{1}$-invariant subsheaf of $\Omega_{/ R}^{q}$. Then for $X \in \mathbf{S m}$,

$$
h_{\mathbf{A}^{1}}^{0}\left(\Omega_{/ R}^{q}\right)(X)=H^{0}\left(\bar{X}, \Omega_{\bar{X} / R}^{q}(\log D)\right),
$$

where $\bar{X}$ is any smooth compactification of $X$ with simple normal crossings divisor $D$ at $\infty$.

Proof. First note that $\widetilde{\Omega^{q}}(\mathcal{X})=\widetilde{\Omega^{q}}\left(X_{1}, \pi^{*} D\right)$, where $\pi: X_{1} \rightarrow X$ is any blowup with centre in $D$, since $(X, D) \cong\left(X_{1}, \pi^{*} D\right)$ in MCor. Let $\mathcal{X}=(X, D)$ be a proper modulus pair with $D_{\text {red }}$ a simple normal crossings divisor. Write $D=\sum_{i} r_{i} \cdot \bar{\eta}_{i}$ with $\eta_{i} \in X^{(1)}$ and set $L_{\eta_{i}}:=\operatorname{Frac}\left(\mathcal{O}_{X, \eta_{i}}^{h}\right)$. Then it is direct to check that we have $c_{L}^{\mathrm{dR}}\left(\rho^{*} a\right) \leq v_{L}(D)$ for all henselian dvf points $\rho$ : Spec $L \rightarrow \mathcal{X}$ if and only if $c_{L_{\eta_{i}}}^{\mathrm{dR}}(a) \leq r_{i}$ for all $i$. Thus the corollary follows from Theorems 6.4 and 4.15(4) and Corollary 4.32 .

\subsection{Rank 1 connections and irregularity}

Lemma 6.9. The homomorphism $\operatorname{dlog}: \mathcal{O}_{X}^{\times} \rightarrow \Omega_{X / R}^{1}, X \in \mathbf{S m}$ induces a morphism $\operatorname{dlog}$ : $\mathcal{O}^{\times} \rightarrow \Omega_{/ R}^{1}$ in $\mathbf{R S C}_{\mathrm{Nis}}$ 
Proof. The proof is similar to the one of Lemma 6.2, except that we have to replace the formula $f_{*} d=d f_{*}$ by $f_{*} \operatorname{dlog}=\operatorname{dlog} \mathrm{Nm}_{Z / X}$, where $f: Z \rightarrow X$ is a finite étale map between smooth schemes.

6.10. Denote by $\operatorname{Conn}^{1}(X)$ the group of isomorphism classes of rank 1 connections on $X \in \mathbf{S m}$, and by $\operatorname{Conn}_{\text {int }}^{1}(X)$ the subgroup of integrable connections. We have canonical group isomorphisms

$$
\operatorname{Conn}^{1}(X) \cong H^{1}\left(X_{\mathrm{Zar}}, \mathcal{O}_{X}^{\times} \stackrel{\operatorname{dlog}}{\longrightarrow} \Omega_{X / k}^{1}\right) \cong H^{0}\left(X,\left(\Omega_{/ k}^{1} / \operatorname{dlog} \mathcal{O}_{X}^{\times}\right)_{\mathrm{Nis}}\right)
$$

and

$$
\operatorname{Conn}_{\mathrm{int}}^{1}(X) \cong H^{1}\left(X_{\mathrm{Zar}}, \mathcal{O}_{X}^{\times} \stackrel{\mathrm{dlog}}{\longrightarrow} Z \Omega_{X / k}^{1}\right) \cong H^{0}\left(X,\left(Z \Omega_{/ k}^{1} / \operatorname{dlog} \mathcal{O}^{\times}\right)_{\mathrm{Nis}}\right) .
$$

Indeed, the first isomorphism is well known (use the fact that the first Zariski cohomology can be computed as Cech cohomology); we show the second as follows: let $\bar{k}^{X}$ be the algebraic closure of $k$ in $k(X)$; we consider it as a constant sheaf on $X$. We obtain the isomorphism

$$
\left[\mathcal{O}_{X}^{\times} /\left(\bar{k}^{X}\right)^{\times} \stackrel{\operatorname{dlog}}{\longrightarrow} \Omega_{X / k}^{1}\right] \cong\left(\Omega_{/ k}^{1} / \operatorname{dlog} \mathcal{O}_{X}^{\times}\right)_{\mathrm{Zar}}[-1]
$$

in the derived category of abelian sheaves on $X_{\mathrm{Zar}}$, and similarly with $Z \Omega_{/ k}^{1}$. Observe that $\Omega_{/ k}^{1}$ and $\mathcal{O}^{\times}$are already Nisnevich sheaves, hence

$$
\left(\Omega_{/ k}^{1} / \operatorname{dlog} \mathcal{O}_{X}^{\times}\right)_{\mathrm{Zar}}=\left(\Omega_{/ k}^{1} / \mathrm{d} \log \mathcal{O}_{X}^{\times}\right)_{\mathrm{Nis}} .
$$

Since $H^{i}\left(X_{\text {Zar }}, \bar{k}^{X}\right)=0$ for all $i \geq 1$, we obtain

$$
H^{1}\left(X_{\mathrm{Zar}}, \mathcal{O}_{X}^{\times} \rightarrow \Omega_{X / k}^{1}\right)=H^{1}\left(X_{\mathrm{Zar}}, \mathcal{O}_{X}^{\times} /\left(\bar{k}^{X}\right)^{\times} \rightarrow \Omega_{X / k}^{1}\right),
$$

and similarly with $Z \Omega_{/ k}^{1}$. This yields the second isomorphism.

By Lemma 6.9 and [45, Theorem 0.1] we obtain

$$
\text { Conn }^{1}, \operatorname{Conn}_{\text {int }}^{1} \in \mathbf{R S C}_{\mathrm{Nis}} \text {. }
$$

For $E \in \operatorname{Conn}^{1}(X)$ we denote by $\omega_{E} \in H^{0}\left(X,\left(\Omega_{/ k}^{1} / d \log \mathcal{O}^{\times}\right)_{\text {Nis }}\right)$ the element corresponding to $E$ under the isomorphism from before.

Set $L \in \Phi$ and let $t \in \mathcal{O}_{L}$ be a local parameter. Recall (for example, from [31, Definition. 1.12]) that the irregularity of $E \in \operatorname{Conn}^{1}(\operatorname{Spec} L) \cong \Omega_{L / k}^{1} / \operatorname{dlog} L^{\times}$is defined by

$$
\operatorname{irr}_{L}(E)=\min \left\{n \geq 0 \mid \omega_{E} \in \operatorname{Im}\left(\frac{1}{t^{n}} \cdot \Omega_{\mathcal{O}_{L} / k}^{1}(\log ) \rightarrow \Omega_{L / k}^{1} / \operatorname{dlog} L^{\times}\right)\right\} .
$$

Theorem 6.11. Notations are as in Section 6.10. The motivic conductor of $E \in$ $\operatorname{Conn}^{1}(L)$ is given by

$$
c_{L}^{\text {Conn }^{1}}(E)= \begin{cases}0 & \text { if } E \text { extends to an } \mathcal{O}_{L} \text {-connection }, \\ \operatorname{irr}_{L}(E)+1 & \text { otherwise. }\end{cases}
$$


Moreover, on $\mathrm{Conn}^{1}$ the motivic conductor restricts to a level 2 conductor, and on $\mathrm{Conn}_{\mathrm{int}}^{1}$ it restricts to a level 1 conductor.

Proof. Set $\mathcal{H}^{1}:=\left(\Omega_{/ k}^{1} / \operatorname{dlog} \mathcal{O}^{\times}\right)_{\text {Nis }}$ and $\mathcal{H}_{\text {int }}^{1}:=\left(Z \Omega_{/ k}^{1} / \operatorname{dlog} \mathcal{O}^{\times}\right)_{\text {Nis }}$. For $a \in \mathcal{H}^{1}(L)$ we define

$$
c_{L}^{\mathrm{irr}}(a):=\min \left\{c_{L}^{\mathrm{dR}}(\tilde{a}) \mid \tilde{a} \in \Omega_{L / k}^{1} \text { lift of } a\right\}
$$

(see Section 6.3 for the definition of $c^{\mathrm{dR}}$ ). It suffices to prove the identity

$$
c^{\mathcal{H}^{1}}=c^{\mathrm{irr}}
$$

for the motivic conductor of $\mathcal{H}^{1}$ and that $\left(c^{\mathrm{irr}}\right)^{\leq 2}$ and $\left(c^{\mathrm{irr}}\right)_{\mid \mathcal{H}_{\mathrm{int}}^{1}}^{\leq 1}$ satisfy $(\mathrm{c} 4)$. It follows directly from Theorem 6.4 and Lemma 4.28 that $c^{\text {irr }}$ satisfies (c1)-(c6), except maybe (c4) and (c5). For (c5), note that given $X \in \mathbf{S m}$, we find by resolution of singularities a compactification $\mathcal{X}=\left(\bar{X}, X_{\infty}\right)$ with $\bar{X} \in \mathbf{S m}$. In particular, for all $x \in \bar{X}$ the local ring $\mathcal{O} \frac{h}{X, x}$ is factorial, and hence so is any of its localisations. Therefore, it follows from the exact sequence

$$
H^{0}\left(Y, \Omega_{Y / k}^{1}\right) \rightarrow \mathcal{H}^{1}(Y) \rightarrow \operatorname{Pic}(Y)
$$

for any integral scheme $Y$ over $k$, that condition (4.28.1) from Lemma 4.28 is satisfied; hence $c^{\text {irr }}$ satisfies (c5). Next we deal with (c4). Take $a \in \mathcal{H}^{1}\left(\mathbf{A}_{X}^{1}\right)$ with

$$
c_{k(x)(t)_{\infty}}^{\mathrm{irr}}\left(a_{x}\right) \leq 1, \quad \text { for all } x \in X \text { with } \operatorname{trdeg}(k(x) / k) \leq 1,
$$

where $a_{x}$ is the restriction of $a$ to $k(x)(t)_{\infty}$. Using the $\mathbf{A}^{1}$-invariance of $X \mapsto H^{i}\left(X, \mathcal{O}_{X}^{\times}\right)$, consider the exact sequence

$$
H^{0}\left(X, \mathcal{O}_{X}^{\times}\right) \stackrel{\text { dlog }}{\longrightarrow} H^{0}\left(\mathbf{A}_{X}^{1}, \Omega_{\mathbf{A}_{X}^{1} / k}^{1}\right) \rightarrow \mathcal{H}^{1}\left(\mathbf{A}_{X}^{1}\right) \rightarrow H^{1}\left(X, \mathcal{O}_{X}^{\times}\right) .
$$

Let $\pi: \mathbf{A}_{X}^{1} \rightarrow X$ be the projection and $i: X \hookrightarrow \mathbf{A}_{X}^{1}$ a section. By sequence (6.11.3) there exists an $\tilde{a} \in H^{0}\left(\mathbf{A}_{X}^{1}, \Omega_{\mathbf{A}_{X}^{1} / k}^{1}\right)$ mapping to $a-\pi^{*} i^{*} a$, and any such lift satisfies formula (6.11.2) with $c^{\text {irr }}$ replaced by $c^{\Omega^{1} / k}$. Thus $\tilde{a} \in H^{0}\left(X, \Omega_{X / k}^{1}\right)$, by $(\mathrm{c} 4)$ for $\left(c^{\Omega^{1} / k}\right)^{\leq 2}$; hence $\left(c^{\text {irr }}\right)^{\leq 2}$ satisfies (c4). Similarly, we prove $(c 4)$ and $(\mathrm{c} 5)$ for $\left(c^{\mathrm{irr}}\right)_{\mid \mathcal{H}_{\text {int }}^{1}}^{\leq 1}$.

Hence $c^{\text {irr }}$ is a semicontinuous conductor, and we obtain $c^{\mathcal{H}^{1}} \leq c^{\text {irr }}$. We now show the other inequality. Set $L \in \Phi$ and let $\sigma: K \hookrightarrow \mathcal{O}_{L}$ be a coefficient field. Denote by $\overline{\mathrm{fil}_{n}} \subset \mathcal{H}^{1}(L)$ the image of $\mathrm{fil}_{n}=\frac{1}{t^{n-1}} \Omega_{\mathcal{O}_{L} / k}^{1}(\log )$. Take $a \in \overline{\mathrm{fil}_{r+1}}$. Similarly as in the proof of Theorem 6.4 (around equation (6.4.4), and with the notation from there), it suffices to show the implication

$$
\left(a, 1-x t^{r}\right)_{L_{x}, \sigma}=0 \text { in } \mathcal{H}^{1}(K(x)) \Rightarrow a \in \overline{\text { fil }_{r}} .
$$

Let $\tilde{a} \in \mathrm{fil}_{r+1}$ be a lift of $a$; write

$$
\tilde{a}=\frac{1}{t^{r}} \alpha+\beta \frac{d t}{t^{r+1}} \bmod \mathrm{fil}_{r},
$$


with $\alpha \in \Omega_{K / k}^{1}$ and $\beta \in K$. Then the left-hand side of equation (6.11.4) is equivalent to

$$
\operatorname{Res}_{t}\left(\tilde{a} \operatorname{dlog}\left(1-x t^{r}\right)\right)=\operatorname{dlog} f \quad \text { for some } f \in K(x)^{\times} .
$$

Computing the residue symbol yields

$$
-r x \alpha+\beta d x=\operatorname{dlog} f \quad \text { in } \Omega_{K(x) / k}^{1} .
$$

We claim this can only happen if $\alpha=\beta=0$. Indeed, first observe that if $h \in K((x))^{\times}$ is a formal Laurent series such that there exists a form $\gamma \in \Omega_{K / k}^{1}$ with $\operatorname{dlog}(h)=$ $x \cdot \gamma$ in $\widehat{\Omega}_{K((x)) / k}^{1}$, then $\gamma=0=\operatorname{d} \log (h)$. Thus equation (6.11.5) implies that $\operatorname{d} \log (f$. $\exp (-\beta x))=0$ in $\widehat{\Omega}_{K((x)) / k}^{1}$. Hence there exists an element $\lambda \in k_{1}$, the algebraic closure of $k$ in $K$, such that

$$
\lambda \cdot \exp (\beta x)=f \in K(x)^{\times},
$$

which is only possible if $\beta=0$; it follows $\alpha=0$. Thus $a \in \overline{\text { fil }_{r}}$, which proves equation (6.11.4) and completes the proof.

Corollary 6.12. Set $X \in \mathbf{S m}$. Then $h_{\mathbf{A}^{1}}^{0}\left(\operatorname{Conn}_{\text {int }}^{1}\right)(X)$ is the group of isomorphism classes of regular singular rank 1 connections on $X$ (see Section 4.30 for notation).

Proof. Let $E \in \operatorname{Conn}_{\text {int }}^{1}(X)$. Then by definition (see [10, II, Definition 4.5]), $E$ is regular singular if and only if $\operatorname{irr}\left(\rho^{*} E\right)=0$, for all henselian $\operatorname{dvf}$ points $\rho: \operatorname{Spec} L \rightarrow X$ with $\operatorname{trdeg}(L / k)=1$. By Theorem 6.11 and Corollary 4.18, this is equivalent to $c_{L}^{\operatorname{Conn}_{\text {int }}^{1}}\left(\rho^{*} a\right) \leq 1$ for all $L$. Thus the statement follows from Corollary 4.32 .

\section{Witt vectors of finite length}

In this section we assume that $k$ is a perfect field of characteristic $p>0$. Denote by $W_{n}$ the ring scheme of $p$-typical Witt vectors of length $n$. We will denote by $W_{n} \mathcal{O}_{X}$ the (Zariski, Nisnevich, étale) sheaf on $X$ defined by $W_{n}$. The restriction of $W_{n}$ to $k$-schemes - which, by abuse of notation, we will again denote by $W_{n}$ - is in particular a smooth commutative group over $k$. Hence $W_{n} \in \mathbf{R S C}_{\mathrm{Nis}}$ (see Section 5.1).

7.1. Let $A$ be a ring. Recall that there is an isomorphism of groups

$$
\begin{gathered}
W_{n}(A) \stackrel{\simeq}{\rightarrow}(1+T A[[T]])^{\times} /\left\{\prod_{s \notin\left\{1, p, \ldots, p^{n-1}\right\}}\left(1-b_{s} T^{s}\right) \mid b_{s} \in A\right\}, \\
\left(a_{0}, \ldots, a_{n-1}\right) \mapsto \prod_{i=0}^{n-1}\left(1-a_{i} T^{p^{i}}\right) .
\end{gathered}
$$

Assume that $A$ is normal and we have an inclusion of rings $A \hookrightarrow B$ making $B$ a finite $A$-module. Then $B[[T]]$ is finite over the normal ring $A[[T]]$, and hence the norm map $\mathrm{Nm}: B[[T]]^{\times} \rightarrow A[[T]]^{\times}$induces a trace $\operatorname{Tr}: W_{n}(B) \rightarrow W_{n}(A)$ (see, for example, [44, Proposition A.9]). 
Now assume that $f: Y \rightarrow X$ is a finite and surjective $k$-morphism, where $X$ is a normal $k$-scheme. Then the local traces glue to give

$$
\operatorname{Tr}_{f}: W_{n}(Y) \rightarrow W_{n}(X) .
$$

Lemma 7.2. In the foregoing situation, $\operatorname{Tr}_{f}=f_{*}: W_{n}(Y) \rightarrow W_{n}(X)$, the map used to define the transfer structure on the group scheme $W_{n}$.

Proof. Set $a \in W_{n}(Y)$ and let $d=\operatorname{deg}(f)$. Recall that the element $f_{*}(a)$ is defined by the composition

$$
X \rightarrow \operatorname{Sym}_{X}^{d} Y \stackrel{\sum^{d} a}{\longrightarrow} W_{n} .
$$

It suffices to check that $\operatorname{Tr}_{f}(a)$ and $f_{*}(a)$ coincide on a dense open subset. Thus we can assume that $X$ is affine integral and $f: Y \rightarrow X$ is finite free. Furthermore, $W_{n}$ is a direct factor of the scheme of big Witt vectors $\mathbb{W}_{p^{n}}$, and $\operatorname{Tr}$ and $f_{*}$ extend to the big Witt vectors. Thus it suffices to show the equality on the big Witt vectors $\mathbb{W}_{r}$ for $r \geq 1$. Let $S_{r}=\operatorname{Spec} k[t] /\left(t^{r+1}\right)$ and denote by $\varepsilon: S=\operatorname{Spec} k \hookrightarrow S_{r}$ the $S$-section. We have the following isomorphism of $S$-group schemes (compare Section (7.1.1)):

$$
\mathbb{W}_{r} \cong \operatorname{Ker}\left(\operatorname{Res}_{S_{r} / S}\left(\mathbf{G}_{m}\right) \stackrel{\varepsilon^{*}}{\rightarrow} \mathbf{G}_{m}\right)
$$

where $\operatorname{Res}_{S_{r} / S}\left(\mathbf{G}_{m}\right)$ denotes the Weil restriction. Denote by $f_{r}: Y_{r} \rightarrow X_{r}$ the base change of $f$ along $S_{r} \rightarrow S$. Set $b \in \mathbb{W}_{r}(Y)$, which we can view as an element in $\operatorname{Res}_{S_{r} / S}\left(\mathbf{G}_{m}\right)(Y)$. Then the image of $f_{*}(b)$ in $\mathbb{W}_{r}(X) \subset \operatorname{Res}_{S_{r} / S}\left(\mathbf{G}_{m}\right)(X)$ is equal to the $S_{r}$-morphism

$$
f_{r *}(b): X_{r} \rightarrow \operatorname{Sym}_{X_{r}}^{d} Y_{r}=X_{r} \times_{X} \operatorname{Sym}_{X}^{d}(Y) \stackrel{\Pi^{d} b}{\longrightarrow} \mathbf{G}_{m, S_{r}} .
$$

Now the statement follows from the fact that $f_{*}=\mathrm{Nm}$ on $\mathbf{G}_{m}$ (see [2, Exp. XVII, Ex $6.3 .18])$.

7.3. Set $L \in \Phi$. Denote by fil ${ }_{j}^{\log } W_{n}(L), j \geq 0$, the Brylinski-Kato filtration [5, 30] - that is,

$$
\begin{aligned}
\text { fil }_{j}^{\log } W_{n}(L) & =\left\{a \in W_{n}(L) \mid[x] \cdot F^{n-1}(a) \in W_{n}\left(\mathcal{O}_{L}\right), \text { all } x \in \mathfrak{m}_{L}^{j}\right\} \\
& =\left\{\left(a_{0}, \ldots, a_{n-1}\right) \in W_{n}(L) \mid p^{n-1-i} v\left(a_{i}\right) \geq-j \text {, all } i\right\},
\end{aligned}
$$

where $[x]$ denotes the Teichmüller lift of $x$ and $F: W_{n}(L) \rightarrow W_{n}(L)$ is the Frobenius, which by contravariant functoriality is induced by the Frobenius of $L$ (or by covariant functoriality by the base change over $\operatorname{Spec} k$ of the Frobenius on the $\operatorname{Spec}\left(\mathbf{F}_{p}\right)$-ring scheme $\left.W_{n}\right)$. We observe that for $s \geq 0$ we have

$$
\mathrm{V}^{s}\left(\mathrm{fil}_{j}^{\log } W_{n}(L)\right) \subset \mathrm{fil}_{j}^{\log } W_{n+s}(L),
$$

where $\mathrm{V}$ is the Verschiebung on the Witt vectors. The nonlog version introduced by Matsuda in $[37,3.1]$ is given (with the conventions from $[35,2.1]$ ) by

$$
\mathrm{fil}_{j} W_{n}(L)=\mathrm{fil}_{j-1}^{\log } W_{n}(L)+\mathrm{V}^{n-r}\left(\mathrm{fil}_{j}^{\log } W_{r}(L)\right), \quad j \geq 1,
$$


where $r=\min \left\{n, \operatorname{ord}_{p}(j)\right\}$. (This is equal to Matsuda's fil $_{j-1}^{\prime} W_{n}(L)$.) Assume $r=$ $\operatorname{ord}_{p}(j)<n$; then

$$
\left(a_{0}, \ldots, a_{n-1}\right) \in \mathrm{fil}_{j} W_{n}(L) \Longleftrightarrow p^{n-1-i} v\left(a_{i}\right) \begin{cases}\geq-j & \text { if } i \neq n-1-r, \\ >-j & \text { otherwise. }\end{cases}
$$

This is the description given in $[32,4.7]$. (There ${ }^{b} \operatorname{fil}_{j} W_{n}(L)$ denotes what we call $\mathrm{fil}_{j} W_{n}(L)$.) We directly check that

$$
\mathrm{F}^{n-1} d\left(\operatorname{fil}_{j} W_{n}(L)\right) \subset \mathfrak{m}_{L}^{-j} \cdot \Omega_{\mathcal{O}_{L}}^{1}
$$

where $\mathrm{F}^{n-1} d$ is the map

$$
\mathrm{F}^{n-1} d: W_{n}(L) \rightarrow \Omega_{L}^{1}, \quad\left(a_{0}, \ldots, a_{n-1}\right) \mapsto \sum_{i=0}^{n-1} a_{i}^{p^{n-1-i}-1} d a_{i} .
$$

7.4. Set $L \in \Phi$. The $F$-saturation of $\mathrm{fil}_{j}^{\log } W_{n}(L)$ and $\mathrm{fil}_{j} W_{n}(L)$ is introduced in [32]:

$$
\mathrm{fil}_{j}^{\log , F} W_{n}(L)=\sum_{r \geq 0} F^{r}\left(\mathrm{fil}_{j}^{\log } W_{n}(L)\right), \quad j \geq 0
$$

and

$$
\mathrm{fil}_{j}^{F} W_{n}(L)=\sum_{r \geq 0} F^{r}\left(\operatorname{fil}_{j} W_{n}(L)\right), \quad j \geq 1 .
$$

Let $\kappa$ be the residue field of $\mathcal{O}_{L}$. Denote by $\kappa[F]$ the noncommutative polynomial ring in the variable $F$ and with coefficients in $\kappa$ with relation $F a=a^{p} F$ in $\kappa[F]$ for $a \in \kappa$. By $[32,4.7]$, there is an injective homomorphism for $j \geq 1$

$$
\bar{\theta}_{j}: \frac{\mathrm{fil}_{j}^{\log , F} W_{n}(L)}{\mathrm{fil}_{j}^{F} W_{n}(L)} \hookrightarrow \kappa[F] \otimes_{\kappa}\left(\frac{\Omega_{\mathcal{O}_{L}}^{1}(\log ) \otimes_{\mathcal{O}_{L}} \mathfrak{m}_{L}^{-j} / \mathfrak{m}_{L}^{-j+1}}{\Omega_{\mathcal{O}_{L}}^{1} \otimes_{\mathcal{O}_{L}} \mathfrak{m}_{L}^{-j} / \mathfrak{m}_{L}^{-j+1}}\right),
$$

induced by (compare formula 7.3.2)

$$
\sum_{r \geq 0} F^{r}\left(a_{r}\right) \mapsto \sum_{r \geq 0}\left(F^{r} \otimes \mathrm{F}^{n-1} d a_{r}\right) .
$$

For $a \in W_{n}(L)$, we define the Brylinski-Kato-Matsuda-Russell conductor $\gamma_{n, L}(a)$ (compare [32, Theorem 8.7]) by

$$
\gamma_{n, L}(a):= \begin{cases}0 & \text { if } a \in W_{n}\left(\mathcal{O}_{L}\right), \\ \min \left\{j \geq 1 \mid a \in \operatorname{fil}_{j}^{F} W_{n}(L)\right\} & \text { otherwise. }\end{cases}
$$

Note that $\operatorname{fil}_{1}^{F} W_{n}(L)=W_{n}\left(\mathcal{O}_{L}\right)$. Thus $\gamma_{n, L}(a)=0$ or $\geq 2$.

Proposition 7.5. The collection

$$
\gamma_{n}=\left\{\gamma_{n, L}: W_{n}(L) \rightarrow \mathbb{N}_{0} \mid L \in \Phi\right\}
$$

is a semicontinuous conductor on $W_{n}$, as is its restriction $\gamma_{n}^{\leq 1}$. 
Proof. Set $\gamma:=\gamma_{n}$. Conditions (c1) and (c2) of Definition 4.3 are clear. For (c3), let $L^{\prime} / L$ be a finite extension of henselian dvfs. Let $e=e\left(L^{\prime} / L\right)$ be the ramification index. Set $a \in W_{n}\left(L^{\prime}\right)$ and define $r:=\gamma_{L^{\prime}}(a)$. We have to show

$$
\operatorname{Tr}(a) \in \operatorname{fil}_{s}^{F} W_{n}(L), \quad \text { with } s:=\left\lceil\frac{r}{e}\right\rceil,
$$

where $\operatorname{Tr}=\operatorname{Tr}_{L^{\prime} / L}$ (see Lemma 7.2). This is immediate if $r=0$. Thus, we can assume $r \geq 2$ and write $a=\sum_{j \geq 0} F^{j}\left(a_{j}\right)$, with $a_{j} \in$ fil $_{r} W_{n}\left(L^{\prime}\right)$. We have $\operatorname{Tr}\left(a_{j}\right) \in$ fil $_{s}^{\log } W_{n}(L)$. Indeed, this follows from

$$
\begin{aligned}
{\left[\mathfrak{m}_{L}^{s}\right] \cdot F^{n-1}\left(\operatorname{Tr}\left(a_{j}\right)\right) } & \subset \operatorname{Tr}\left(\left[\mathfrak{m}_{L^{\prime}}^{s e}\right] \cdot F^{n-1}\left(a_{j}\right)\right) \\
& \subset \operatorname{Tr}\left(\left[\mathfrak{m}_{L^{\prime}}^{r}\right] \cdot F^{n-1}\left(a_{j}\right)\right) \\
& \subset \operatorname{Tr}\left(W_{n}\left(\mathcal{O}_{L^{\prime}}\right)\right) \subset W_{n}\left(\mathcal{O}_{L}\right)
\end{aligned}
$$

where for $b \in W_{n}(L)$ we denote $\left[\mathfrak{m}_{L}^{j}\right] \cdot b:=\left\{[x] \cdot b \mid x \in \mathfrak{m}_{L}^{j}\right\}$. Hence

$$
\operatorname{Tr}(a)=\sum_{j} F^{j}\left(\operatorname{Tr}\left(a_{j}\right)\right) \in \mathrm{fil}_{s}^{\log , F} W_{n}(L) .
$$

By the injectivity of $\bar{\theta}_{s}$ in formula (7.4.3), it suffices to show

$$
\mathfrak{m}_{L}^{s} \cdot F^{n-1} d \operatorname{Tr}\left(a_{j}\right) \in \Omega_{\mathcal{O}_{L}}^{1}, \quad \text { all } j \geq 0 .
$$

By [44, Theorem 2.6], the trace Tr extends to a trace between the de Rham-Witt complexes $\operatorname{Tr}: W_{n} \Omega_{L^{\prime}} \rightarrow W_{n} \Omega_{L}$ which is compatible with the differential and Frobenius, is $W_{n} \Omega_{L}$-linear and equals the classical trace on Kähler differentials for $n=1$. We obtain

$$
\begin{aligned}
\mathfrak{m}_{L}^{s} \cdot F^{n-1} d \operatorname{Tr}(a) & =\mathfrak{m}_{L}^{s} \cdot \operatorname{Tr}\left(F^{n-1} d a\right) \\
& \subset \mathfrak{m}_{L}^{s} \cdot \operatorname{Tr}\left(\mathfrak{m}_{L^{\prime}}^{-r} \cdot \Omega_{\mathcal{O}_{L^{\prime}}}^{1}\right), \quad a \in \operatorname{fil}_{r} W_{n}\left(L^{\prime}\right) \text { (see formula }(7.3 .2) \\
& \subset \operatorname{Tr}\left(\mathfrak{m}_{L^{\prime}}^{e s-r} \cdot \Omega_{\mathcal{O}_{L^{\prime}}}^{1}\right) \\
& \subset \operatorname{Tr}\left(\Omega_{\mathcal{O}_{L^{\prime}}}^{1}\right) \subset \Omega_{\mathcal{O}_{L}}^{1}
\end{aligned}
$$

This completes the proof for $(\mathrm{c} 3)$.

Next we show that the restriction of $\gamma$ to $\Phi_{\leq 1}$ satisfies (c4). Set $X \in \mathbf{S m}$ and $a \in$ $W_{n}\left(\mathbf{A}_{X}^{1}\right)$ with

$$
\gamma_{k(x)(t)_{\infty}}\left(\rho_{x}^{*} a\right) \leq 1
$$

for closed points $x \in X$, where $k(x)(t)_{\infty}=\operatorname{Frac}\left(\mathcal{O}_{\mathbf{P}_{x}^{1}, \infty}^{h}\right)$. We have to show $a \in W_{n}(X)$. We may assume $X=\operatorname{Spec} A$, and thus $a \in W_{n}(A[t])$. If $a$ is not constant, then we find a closed point $x \in X$ such that the image of $a$ in $W_{n}(k(x)[t])$ is not constant. Hence $a_{k(x)(t)_{\infty}} \notin W_{n}\left(\mathcal{O}_{k(x)(t)_{\infty}}\right)$ - that is, $\gamma\left(a_{k(x)(t)_{\infty}}\right) \geq 2$, contradicting assumption (7.5.3).

(c5). Set $X \in \mathbf{S m}$ and $a \in W_{n}(X)=H^{0}\left(X, W_{n} \mathcal{O}_{X}\right)$. Let $\mathcal{X}=\left(\bar{X}, X_{\infty}\right)$ be a proper modulus pair with $X=\bar{X} \backslash\left|X_{\infty}\right|$. For an effective Cartier divisor $E$ on $\bar{X}$, denote by $W_{n} \mathcal{O}_{\bar{X}}(E)$ the invertible subsheaf of $j_{*} W_{n} \mathcal{O}_{\bar{X} \backslash|E|}$ corresponding to the image of 
$\left[\mathcal{O}_{\bar{X}}(E)\right] \in H_{\text {ét }}^{1}\left(\bar{X}, \mathcal{O}_{\bar{X}}^{\times}\right)$in $H_{\text {ét }}^{1}\left(\bar{X}, W_{n} \mathcal{O}_{\bar{X}}^{\times}\right)$under the map induced by the Teichmüller lift. If $e$ is an equation for $E$ at $x \in \bar{X}$, then $W_{n} \mathcal{O}_{\bar{X}, x}(E)=W_{n} \mathcal{O}_{\bar{X}, x} \cdot \frac{1}{[e]}$. There exists an integer $N$ such that $a \in H^{0}\left(\bar{X}, W_{n} \mathcal{O}_{\bar{X}}\left(N \cdot X_{\infty}\right)\right)$.

Claim 7.5.1. $\left(\bar{X}, r X_{\infty}\right)$ satisfies (c5) for any $r>p^{n-1} N$.

Indeed, let $\rho: \operatorname{Spec} L \rightarrow \mathcal{X}$ be a henselian dvf point. Assume that the closed point $s \in S_{L}$ maps into $X_{\infty}$ and let $f \in \mathcal{O}_{\bar{X}, \bar{\rho}(s)}$ be a local equation for $X_{\infty}$. Let $m=v_{L}(f)$. For $r>p^{n-1} N$, we find $\left[\mathfrak{m}_{L}^{r m-1}\right] \cdot F^{n-1}(a) \in W_{n}\left(\mathcal{O}_{L}\right)$; hence (see Section 7.3)

$$
a \in \operatorname{fil}_{r m-1}^{\log } W_{n}(L) \subset \operatorname{fil}_{r m} W_{n}(L) \subset \operatorname{fil}_{r m}^{F} W_{n}(L) ;
$$

that is, $\gamma_{L}\left(\rho^{*} a\right) \leq r m=v_{L}\left(r \cdot X_{\infty}\right)$, proving Claim 7.5.1.

Finally we deal with (c6). Set $X \in \mathbf{S m}$ and let $Z \subset X$ be a smooth prime divisor with generic point $z$. Let $K=\operatorname{Frac}\left(\mathcal{O}_{X, z}^{h}\right)$. Set $a \in W_{n}(X \backslash Z)$. Assume $a_{K} \in \mathrm{fil}_{j}^{F} W_{n}(K)$, $j \geq 2$. Then there exists an affine Nisnevich neighbourhood $U=\operatorname{Spec} A \rightarrow X$ of $z \operatorname{such}$ that $Z_{U}=\operatorname{div}(t)$ on $U$ and $a_{U}=\sum_{s \geq 0} F^{s}\left(a_{s}+\mathrm{V}^{n-r}\left(b_{s}\right)\right)$, where $r=\min \left\{\operatorname{ord}_{p}(j), n\right\}$ and $a_{s} \in W_{n}(A[1 / t]), b_{s} \in W_{r}(A[1 / t])$, with

$$
[t]^{j-1} \cdot F^{n-1}\left(a_{s}\right) \in W_{n}(A), \quad[t]^{j} \cdot F^{r-1}\left(b_{s}\right) \in W_{r}(A) .
$$

Let $(\bar{Y}, \bar{Z}+\Sigma)$ be a compactification of $(U, Z)$ with $\bar{Z}_{\mid U}=Z$ and $\bar{Y}$ normal. Let $\bar{Y}=\cup V_{i}$ be an open covering such that $V_{i}=\operatorname{Spec} B_{i}, \Sigma_{\mid V_{i}}=\operatorname{Div}\left(f_{i}\right)$ and $\bar{Z}_{\mid V_{i}}=\operatorname{Div}\left(\tau_{i}\right)$, with $\tau_{i}, f_{i} \in B_{i}$. Note that $\operatorname{Spec} B_{i}\left[1 / f_{i}\right] \subset U$ is open for all $i$. Hence, in $B_{i}\left[1 / f_{i}\right]$ we can write $t=\tau_{i} e_{i}$, with $e_{i} \in\left(B_{i}\left[1 / f_{i}\right]\right)^{\times}$. Let $E_{i}$ be the Cartier divisor on $V_{i}$ defined by $e_{i}$. We have $\left|E_{i}\right| \subset\left|\Sigma_{\mid V_{i}}\right|$. By Lemma 6.5 , there exists $N_{1} \geq 0$ such that $f_{i}^{N_{1}} / e_{i} \in B_{i}$ for all $i$. By formula (7.5.4), there exists an $N_{2} \geq 0$ such that for all $i$ and all $s$,

$$
\left[f_{i}\right]^{N_{2}}[t]^{j-1} \cdot F^{n-1}\left(a_{s}\right) \in W_{n}\left(B_{i}\right), \quad\left[f_{i}\right]^{N_{2}}[t]^{j} \cdot F^{r-1}\left(b_{s}\right) \in W_{r}\left(B_{i}\right) .
$$

Choose $N \geq j \cdot N_{1}+N_{2}$ such that $p^{n} \mid N$. We obtain for all $i$

$$
\left[\tau_{i}\right]^{j-1}\left[f_{i}\right]^{N-1} \cdot F^{n-1}\left(a_{s}\right) \in W_{n}\left(B_{i}\right), \quad\left[\tau_{i}\right]^{j}\left[f_{i}\right]^{N} \cdot F^{r-1}\left(b_{s}\right) \in W_{r}\left(B_{i}\right) .
$$

Set $\rho: \operatorname{Spec} L \rightarrow U, L \in \Phi$. Assume that the closed point of $\operatorname{Spec} \mathcal{O}_{L}$ maps into $|\bar{Z}+\Sigma|$. Then it follows that

$$
\rho^{*} a_{s} \in \mathrm{fil}_{v_{L}((j-1) \cdot \bar{Z}+(N-1) \cdot \Sigma)}^{\log } W_{n}(K) \subset \mathrm{fil}_{v_{L}(j \cdot \bar{Z}+N \cdot \Sigma)} W_{n}(K)
$$

and

$$
\rho^{*} b_{s} \in \mathrm{fil}_{v_{L}(j \cdot \bar{Z}+N \cdot \Sigma)}^{\log } W_{r}(K) .
$$

By the choice of $N$ we have

$$
r_{0}:=\min \left\{\operatorname{ord}_{p}\left(v_{L}(j \cdot \bar{Z}+N \cdot \Sigma)\right), n\right\} \geq r=\min \left\{\operatorname{ord}_{p}(j), n\right\} ;
$$

hence

$$
\mathrm{V}^{n-r}\left(\rho^{*} b_{s}\right) \in \mathrm{V}^{n-r_{0}} \operatorname{fil}_{v_{L}(j \cdot \bar{Z}+N \cdot \Sigma)}^{\log } W_{r_{0}}(K) \subset \operatorname{fil}_{v_{L}(j \cdot \bar{Z}+N \cdot \Sigma)} W_{n}(K)
$$


Running over all $\rho: \operatorname{Spec} L \rightarrow U$ yields

$$
\gamma_{\bar{Y}}(a) \leq j \cdot \bar{Z}+N \cdot \Sigma .
$$

This proves (c6) and completes the proof of the proposition.

Proposition 7.5 gives $c^{W_{n}} \leq \gamma_{n}$, by Corollary 4.24. We show later in Theorem 7.20 that equality holds using symbol computations. If we restrict to $\operatorname{trdeg}(L / k)=1$ and $k$ is infinite, this follows, for example, from [32, Proposition 6.4, (3)]. To handle the case of higher transcendence degree, we need some preparations. We start by identifying the local symbol for $W_{n}$ on regular projective curves over function fields.

7.6. Set $X \in \mathbf{S m}$. We denote by $W_{n} \Omega_{X}^{\bullet}$ the de Rham-Witt complex of length $n$ on $X$ [22]. By [27, Corollary 3.2.5] we have $W_{n} \Omega^{q} \in \mathbf{R S C}_{\mathrm{Nis}}$. See also [15] and [7] for details on how to define the transfer structure. If $f: X \rightarrow Y$ is a morphism in $\mathbf{S m}$, then the morphism

$$
\Gamma_{f}^{*}=f^{*}: W_{n} \Omega^{q}(Y) \rightarrow W_{n} \Omega^{q}(X)
$$

induced by its graph $\Gamma_{f} \in \operatorname{Cor}(X, Y)$ is the natural pullback morphism induced by the functoriality of the de Rham-Witt complex. If $f$ is finite and surjective, then the transpose of the graph defines an element $\Gamma_{f}^{t} \in \mathbf{C o r}(Y, X)$ and $\Gamma_{f}^{t *}=f_{*}$, where $f_{*}$ is the push-forward defined using duality theory.

\section{Lemma 7.7.}

(1) The restriction, Verschiebung, Frobenius and differential (which are part of the structure of the de Rham-Witt complex) define morphisms in $\mathbf{R S C}_{\mathrm{Nis}}$ :

$$
\begin{array}{ll}
R: W_{n+1} \Omega^{q} \rightarrow W_{n} \Omega^{q}, & \mathrm{~V}: W_{n} \Omega^{q} \rightarrow W_{n+1} \Omega^{q}, \\
F: W_{n+1} \Omega^{q} \rightarrow W_{n} \Omega^{q}, & d: W_{n} \Omega^{q} \rightarrow W_{n} \Omega^{q+1} .
\end{array}
$$

(2) Let $W_{n}$ be the algebraic group of Witt vectors of length $n$ considered as a presheaf on $\mathbf{S m}$. Then there is a unique structure of presheaf with transfers on $W_{n}$, for all $n$, which is unique with the following properties:

(a) the restriction $R: W_{n+1} \rightarrow W_{n}$ is compatible with the transfer structure for all $n$;

(b) if $f: X \rightarrow Y$ is a morphism in $\mathbf{S m}$ with graph $\Gamma_{f} \in \operatorname{Cor}(X, Y)$, then $\Gamma_{f}^{*}$ : $W_{n}(Y) \rightarrow W_{n}(X)$ is the pullback from the presheaf structure.

In particular, the Nisnevich sheaf with transfers $W_{n} \Omega^{0}=W_{n} \mathcal{O}$ from Section $\% .6$ coincides with the Nisnevich sheaf with transfers defined by the algebraic group $W_{n}$ [27, Corollary 3.2.5].

Proof. (1). We have to show that if $\alpha \in \operatorname{Cor}(X, Y)$ is a finite correspondence, then the following morphisms are equal on $H^{0}\left(Y, W_{n} \Omega_{Y}^{q}\right)$ :

$$
\alpha^{*} R=R \alpha^{*}, \quad \alpha^{*} V=V \alpha^{*}, \quad \alpha^{*} F=F \alpha^{*}, \quad \alpha^{*} d=d \alpha^{*} .
$$

This follows from [7, Proof of Proposition 3.5.4]. 
(2). The existence of such a transfer structure follows, for example, from Section 7.6. The last part of the statement follows because the two transfer structures satisfy (2a) and $(2 \mathrm{~b})$.

It remains to prove the uniqueness. Assume we have two transfer actions on $W_{n}$ with (2a) and (2b). For a finite correspondence $\alpha \in \operatorname{Cor}(X, Y)$, denote by $\alpha^{*}, \alpha^{\star}$ : $W_{n}(Y) \rightarrow W_{n}(X)$ the two actions. We have to show that they are equal. Let $f: X \rightarrow Y$ be a morphism. By assumption, we have $\Gamma_{f}^{*}=\Gamma_{t}^{\star}=: f^{*}$; if $f$ is finite and and surjective, we set $f_{*}:=\left(\Gamma_{f}^{t}\right)^{*}$ and $f_{\star}:=\left(\Gamma_{f}^{t}\right)^{\star}$. In general, for $\alpha$ as before, we want to show $\alpha^{*}=\alpha^{\star}$. It suffices to check this after shrinking $X$ around its generic points. Hence, we can assume that $X$ is connected and $\alpha=Z \subset X \times Y$ with $Z$ smooth, integral and finite free over $X$. Denote by $f: Z \rightarrow X$ and $g: Z \rightarrow Y$ the maps induced by the projections. Then $\alpha^{\star}=f_{\star} g^{*}$ and $\alpha^{*}=f_{*} g^{*}$. It remains to show $f_{\star}=f_{*}$. We may shrink $X$ further and hence assume that $f: Z=\operatorname{Spec} L \rightarrow X=\operatorname{Spec} K$ is induced by a finite field extension $L / K$ of function fields over $k$. By transitivity, it suffices to consider the two cases where $L / K$ is either separable or purely inseparable of degree $p$.

Case 1: $L / K$ separable. Let $K^{\prime} / K$ be a Galois hull of $L / K$ and set $X^{\prime}=\operatorname{Spec} K^{\prime}$. We obtain the cartesian diagram

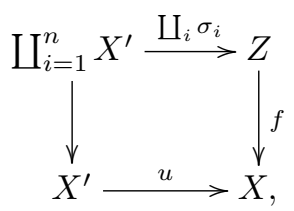

where the vertical map on the left is induced by the universal property of the coproduct from the identity on $X^{\prime}, u$ is induced by the inclusion $K \hookrightarrow K^{\prime}$ and the $\sigma_{i}: X^{\prime} \rightarrow Z$, $i=1, \ldots, n$, are induced by all the $K$-embeddings $L \hookrightarrow K^{\prime}$. For $a \in W_{n}(L)$ we obtain

$$
u^{*} f_{*} a=\left(\Gamma_{f}^{t} \circ \Gamma_{u}\right)^{*}=\sum_{i} \Gamma_{\sigma_{i}}^{*}
$$

and similarly with $u^{*} f_{\star}$. Thus $u^{*} f_{*}=u^{*} f_{\star}$, and since $u^{*}: W_{n}(K) \hookrightarrow W_{n}\left(K^{\prime}\right)$ is injective, we have proven the claim in this case.

Case 2: $L / K$ purely inseparable of degree $p$. In this case we have

$$
f_{*} f^{*}(-)=[L: K] \cdot(-)=p \cdot(-)=f_{\star} f^{\star}(-) \text { on } W_{n}(X) .
$$

Let $p: W_{n} \rightarrow W_{n+1}$ be the map lift-and-multiply-by-p; thus it sends a Witt vector $\left(a_{0}, \ldots, a_{n-1}\right)$ in $W_{n}(A)$, where $A$ is some $\mathbf{F}_{p}$-algebra, to $\left(0, a_{0}^{p}, \ldots, a_{n-1}^{p}\right)$. Set $b \in W_{n}(L)$. Clearly we find an element $a \in W_{n+1}(K)$ such that $f^{*} a=p(b)$. We obtain

$$
\underline{p}\left(f_{*} b\right) \stackrel{(2 a)}{=} f_{*} \underline{p}(b)=f_{*}\left(f^{*} a\right) \stackrel{\text { equation }}{=}{ }^{(7.7 .1)} p \cdot a=\underline{p} R(a) .
$$

The same computation works for $f_{\star} b$. Thus $p\left(f_{\star} b\right)=p\left(f_{\star} b\right)$, and the claim follows from the injectivity of $\underline{p}$. 
Lemma 7.8. Let $f: Y \rightarrow X$ be a finite and surjective morphism in $\mathbf{S m}$. Then for all $u \in H^{0}\left(Y, \mathcal{O}_{Y}^{\times}\right)$and all $n \geq 1$ we have

$$
f_{*} \operatorname{dlog}[u]=\operatorname{dlog}\left[\operatorname{Nm}_{Y / X}(u)\right] \quad \text { in } H^{0}\left(X, W_{n} \Omega_{X}^{1}\right),
$$

where $\mathrm{Nm}_{Y / X}: f_{*} \mathcal{O}_{Y}^{\times} \rightarrow \mathcal{O}_{X}^{\times}$is the usual norm.

Proof. Note that $f$ is flat by [38, Theorem 23.1], hence also finite locally free, so that $\mathrm{Nm}_{Y / X}$ is defined. It suffices to prove the equality after shrinking $X$ around its generic points. Thus we can assume that $f$ corresponds to a finite field extension $L / K$. By transitivity, it suffices to consider the cases where $L / K$ is separable or purely inseparable of degree $p$.

Case 1: $L / K$ finite separable. We have $W_{n} \Omega_{L}^{q}=W_{n}(L) \otimes_{W_{n}(K)} W_{n} \Omega_{K}^{q}[22$, I, Proposition 1.14]. By the projection formula and Lemma 7.7(2), we have $f_{*}=\operatorname{Tr}_{L / K} \otimes$ id. Let $K^{\text {sep }}$ be a separable closure of $K$. Note that $W_{n}(K) \rightarrow W_{n}\left(K^{\text {sep }}\right)$ is faithfully flat (since it is ind-étale and $\operatorname{Spec} W_{n}(K)$ is one point). Hence by étale base change and fppf descent, the natural map $W_{n} \Omega_{K}^{1} \rightarrow W_{n} \Omega_{K^{\text {sep }}}^{1}$ is injective. Thus it suffices to check the equality in $W_{n} \Omega_{K^{\text {sep }}}^{1}$. Let $\sigma_{1}, \ldots, \sigma_{r}: L \hookrightarrow K^{\text {sep }}$ be all $K$-embeddings; then by the foregoing we have in $W_{n} \Omega_{K^{\text {sep }}}^{1}$

$$
f_{*} \operatorname{dlog}[u]=\sum_{i=1}^{r} \sigma_{i}(\operatorname{dlog}[u])=\operatorname{dlog}\left[\prod_{i=1}^{r} \sigma_{i}(u)\right]=\operatorname{dlog}\left[\operatorname{Nm}_{L / K}(u)\right] .
$$

Case 2: $L / K$ purely inseparable of degree $p$. We have $\operatorname{Nm}_{L / K}(u)=u^{p} \in K$. Since the map lift-and-multiply-by-p, $\underline{p}: W_{n} \Omega_{K}^{1} \rightarrow W_{n+1} \Omega_{K}^{1}$, is injective by [22, I, Proposition 3.4] and commutes with $f_{*}$, the statement follows from the following equality in $W_{n+1} \Omega_{K}^{1}$ :

$$
\underline{p}\left(f_{*} \operatorname{d} \log [u]_{n}\right)=f_{*} \operatorname{dlog}\left[u^{p}\right]_{n+1}=f_{*}(1) \cdot \operatorname{dlog}\left[u^{p}\right]_{n+1}=\underline{p} \operatorname{dlog}\left[\operatorname{Nm}_{L / K}(u)\right]_{n} .
$$

This completes the proof of the lemma.

7.9. Let $A$ be a ring of characteristic $p$ and set $B:=A[[t]]\left[\frac{1}{t}\right]$. Recall from $[29, \S 2.2$, Proposition 3] and [43, Proposition 2.12] that there is a residue map

$$
\operatorname{Res}_{t}: W_{n} \Omega_{B}^{*} \rightarrow W_{n} \Omega_{A}^{*-1}
$$

which is $W_{n} \Omega_{A}^{*}$-linear (where we consider the left-module structures), commutes with $R$, $F, V$, and $d$, is zero on $W_{n} \Omega_{A[[t]]}^{*}$ and satisfies the equality $\operatorname{Res}_{t}(\alpha \operatorname{dlog}[t])=\alpha(0)$, for $\alpha \in W_{n} \Omega_{A[[t]]}^{*}$.

Let $K$ be a function field over $k$ and $C$ be a regular projective connected curve over $K$ with function field $E=K(C)$. Recall from [44, Definition-Proposition 1] that the residue map

$$
\operatorname{Res}_{C / K, x}: W_{n} \Omega_{E}^{*} \rightarrow W_{n} \Omega_{K}^{*-1}
$$

at a closed point $x \in C$ is defined as follows: by a result of Hübl and Kunz we find an integer $m_{0} \geq 0$ such that for all $m \geq m_{0}$, the curve $C_{m}:=\operatorname{Spec}\left(\mathcal{O}_{C} \cap K\left(E^{p^{m}}\right)\right)$ is smooth over $K$ and, if $x_{m}$ denotes the image of $x$ under the finite homeomorphism $C \rightarrow C_{m}$, then the residue field $K_{m}:=K\left(x_{m}\right)$ is separable over $K$. Hence $\mathcal{O}_{C_{m}, x_{m}}^{h}$ has a unique 
coefficient field containing $K$, which we identify with $K_{m}$. Set $E_{m}:=K\left(C_{m}\right)=K\left(E^{p^{m}}\right)$. The choice of a local parameter $t \in \mathcal{O}_{C_{m}, x_{m}}$ yields a canonical inclusion $E_{m} \hookrightarrow K_{m}((t))$. We define $\operatorname{Res}_{C / K, x}$ as the composition

$$
W_{n} \Omega_{E}^{*} \stackrel{\operatorname{Tr}_{E / E_{m}}}{\longrightarrow} W_{n} \Omega_{E_{m}}^{*} \hookrightarrow W_{n} \Omega_{K_{m}((t))}^{*} \stackrel{\text { formula (7.9.1) }}{\longrightarrow} W_{n} \Omega_{K_{m}}^{*} \stackrel{\operatorname{Tr}_{K_{m} / K}}{\longrightarrow} W_{n} \Omega_{K}^{*-1} .
$$

(Here we should observe that if $\pi: \operatorname{Spec} L \rightarrow \operatorname{Spec} K$ is a finite extension, then the trace $\operatorname{Tr}_{L / K}: W_{n} \Omega_{L}^{q} \rightarrow W_{n} \Omega_{K}^{q}$ from [43, Theorem 2.6] is equal to the push-forward $\pi_{*}$ from Section 7.6. Indeed, in the case $q=0$ this follows from Lemmas 7.7(2) and 7.2; by transitivity, the general case is reduced to a simple extension $L=K[a]$, in which case it follows from the facts that both maps commute with $V, F, d$ and satisfy a projection formula, and from the equality $[a]^{i-1} d[a]=i_{0}^{-1} F^{e} d[a]^{i_{0}}$, where $i=p^{e} i_{0} \geq 1$ with $\left(i_{0}, p\right)=1$.)

Remark 7.10. In [43, 2.] and [44], where the trace and the residue symbol mentioned earlier are constructed, it is always assumed that the characteristic is not 2. The reason for this that the structure theorem by Hesselholt and Madsen on which those sources rely was only known for $\mathbb{Z}_{(p)}$-algebras, with $p$ odd, at that time. This theorem is used in a proposition and a lemma from those sources which are needed to define the trace and the formal residue symbol, respectively. However, the Hesselholt-Madsen theorem is also available for $\mathbb{Z}_{(2)}$-algebras by [9, 4.2], hence all the results from [44, 43] extend to the case $p=2$.

Lemma 7.11. Let $C / K$ and $x \in C$ be as in Section 7.9. Then the corresponding local symbol of $W_{n} \Omega^{q}$ (see Section 4.34) is given by

$$
(a, f)_{C / K, x}=\operatorname{Res}_{C / K, x}(\alpha \cdot \operatorname{dlog}[f]), \quad \alpha \in W_{n} \Omega_{K(C)}^{q}, f \in K(C)^{\times},
$$

where $[f]=(f, 0, \ldots, 0) \in W_{n}(K(C))$.

In particular, if $L \in \Phi$ with coefficient field $\sigma: K \hookrightarrow \mathcal{O}_{L}$ and local parameter $t \in \mathcal{O}_{L}$, then the local symbol $(-,-)_{L, \sigma}: W_{n} \Omega_{L}^{q} \times L^{\times} \rightarrow W_{n} \Omega_{K}^{q}$ (see Section 4.37) is given by the composition

$$
W_{n} \Omega_{L}^{q} \times L^{\times} \stackrel{\hat{\sigma} \wedge \text { dlog } \circ[-] \circ \hat{\sigma}}{\longrightarrow} W_{n} \widehat{\Omega}_{K((t))}^{q+1} \stackrel{\operatorname{Res}_{t}}{\longrightarrow} W_{n} \Omega_{K}^{q},
$$

where we denote by $\hat{\sigma}: L \hookrightarrow K((t))$ the canonical inclusion.

Proof. We have to show that the family of maps $\left\{\operatorname{Res}_{C / K, x}(-\cdot \operatorname{dlog}[-])\right\}_{x}$ with $x$ running through all the closed points of $C$ satisfies properties (LS1)-(LS4) from Section 4.34. (LS1) (linearity) is clear, and since we can choose the modulus $D$ for (LS3) as large as we want, this condition too is clear, from Lemma 7.13. (LS4) (the reciprocity law) holds by [44, Theorem 2] (see also Remark 7.10). It remains to show (LS2) - that is,

$$
\operatorname{Res}_{C / K, x}(\alpha \operatorname{dlog}(f))=v_{x}(f) \operatorname{Tr}_{K(x) / K}(\alpha(x)), \quad \alpha \in W_{n} \Omega_{C, x}^{q} .
$$

To this end, choose $m$ as in Section 7.9. Then $K(x) / K\left(x_{m}\right)$ is purely inseparable of degree, say, $p^{s}$, and we can write

$$
\left[E: E_{m}\right]=p^{s+e}
$$


where $p^{e}$ is the ramification index of $x / x_{m}$. Denote by $\underline{p}^{s}: W_{n} \Omega^{q} \rightarrow W_{n+s} \Omega^{q}$ the map lift-and-multiply-by-p $p^{s}$; it is injective, by [22, I, Proposition 3.4]. Denote by $\sigma: K_{m}:=$ $K\left(x_{m}\right) \hookrightarrow \mathcal{O}_{C_{m}, x_{m}}^{h} \hookrightarrow \mathcal{O}_{C, x}^{h}$ the inclusion of the coefficient field. By [44, Theorem 2.6(iii)], there exists a $\beta \in W_{n+s} \Omega_{K_{m}}^{q}$ mapping to $\underline{p}^{s} \alpha(x) \in W_{n+s} \Omega_{K(x)}^{q}$ and we have

$$
\operatorname{Tr}_{K(x) / K_{m}}(\alpha(x))=R^{s}(\beta) \text {. }
$$

By the choice of $\beta$, we have

$$
\underline{p}^{s}(\alpha)-\sigma(\beta) \in \operatorname{Ker}\left(W_{n+s} \Omega_{\mathcal{O}_{C, x}^{h}}^{q} \rightarrow W_{n+s} \Omega_{K(x)}^{q}\right) .
$$

Since the kernel is the differential graded ideal generated by $W_{n+s}\left(\mathfrak{m}_{x}\right)$, we obtain in $W_{n+s} \Omega_{K}^{q}$

$$
\begin{aligned}
& \underline{p}^{s} \operatorname{Res}_{C / K, x}(\alpha \operatorname{dlog}[f])=\operatorname{Res}_{C / K, x}\left(\underline{p}^{s}(\alpha \operatorname{dlog}[f])\right) \\
& =\operatorname{Res}_{C / K, x}(\sigma(\beta) \operatorname{dlog}[f]) \\
& \left.=\operatorname{Res}_{C_{m} / K, x_{m}}\left(\operatorname{Tr}_{E / E_{m}}(\sigma(\beta) \operatorname{dlog}[f])\right)\right) \text {, } \\
& =\operatorname{Res}_{C_{m} / K, x_{m}}\left(\beta \operatorname{dlog} \mathrm{Nm}_{E / E_{m}}[f]\right) \text {, } \\
& \left.=v_{x_{m}}\left(\mathrm{Nm}_{E / E_{m}}(f)\right)\right) \cdot \operatorname{Tr}_{K_{m} / K}(\beta) \\
& =\left[K(x): K\left(x_{m}\right)\right] \cdot v_{x}(f) \cdot \operatorname{Tr}_{K_{m} / K}(\beta) \\
& =v_{x}(f) \cdot \underline{p}^{s} \operatorname{Tr}_{K_{m} / K}\left(R^{s}(\beta)\right) \\
& =v_{x}(f) \cdot \underline{p^{s}} \operatorname{Tr}_{K(x) / K}(\alpha(x)),
\end{aligned}
$$

Here the first equality follows from the fact that $\operatorname{Res}_{C / K, x}$ commutes with the restriction $R$. (This follows from the definition and the fact that Res from formula (7.9.1) and Tr commute with $R$; for the latter, see, for example, Lemma 7.7(1).) The statement follows from the injectivity of $\underline{p}^{s}$.

7.12. Let $A$ be a $\mathbb{Z}_{(p)}$-algebra. For an $A$-algebra $B$ we denote by $W_{n} \Omega_{B / A}^{\bullet}$ the relative de Rham-Witt complex of Langer and Zink [36]. It is equipped with R, F, V, $d$ as usual. If $B[x]$ is the polynomial ring with coefficients in $B$, we denote by $I_{r} \subset W_{n} \Omega_{B[x] / A}^{\bullet}$ the differential graded ideal generated by $W_{n}\left(x^{r} B[x]\right)$. We define the $x$-adic completion of $W_{n} \Omega_{B[x] / A}^{\bullet}$ to be

$$
W_{n} \widehat{\Omega}_{B[[x]] / A}^{\bullet}:=\lim _{r} W_{n} \Omega_{B[x] / A}^{\bullet} / I_{r} .
$$

Note that $W_{n} \Omega_{B[x] / A}^{\bullet} / I_{r}=W_{n} \Omega_{\left(B[x] /\left(x^{r}\right)\right) / A}^{\bullet}$ (see [14, Lemma 2.4]). In particular, $W_{n} \widehat{\Omega}_{B[[x]] / A}^{\bullet}$ is a $W_{n}(B[[x]])=\lim _{r} W_{n}\left(B[x] /\left(x^{r}\right)\right)$-module.

Lemma 7.13. The following equality holds in $W_{n} \widehat{\Omega}_{\mathbb{Z}_{(p)}[[x]] / \mathbb{Z}_{(p)}}$ :

$$
-\mathrm{d} \log [1-x]=\sum_{i \geq 0}[x]^{i} d[x]+\sum_{s=1}^{n-1} \sum_{(j, p)=1} \frac{1}{j} d \mathrm{~V}^{s}\left([x]^{j}\right) .
$$


Proof. We prove this by induction over $n$. The case where $n=1$ is clear. Assume $n \geq 2$. By [36, Corollary 2.13] we find unique elements $a_{i} \in W_{n}\left(\mathbb{Z}_{(p)}\right)$ and $b_{s, j} \in W_{n-s}\left(\mathbb{Z}_{(p)}\right)$ such that

$$
-\operatorname{dlog}[1-x]=\sum_{i \geq 0} a_{i}[x]^{i} d[x]+\sum_{s=1}^{n-1} \sum_{(j, p)=1} d \mathrm{~V}^{s}\left(b_{s, j}[x]^{j}\right) .
$$

Applying $\mathrm{F}^{n-1}$, we obtain in $\widehat{\Omega}_{\mathbb{Z}_{(p)}[[x]] / \mathbb{Z}_{(p)}}^{1}$

$$
\begin{aligned}
-\operatorname{dlog}(1-x) & =\sum_{k \geq 0} x^{k} d x \\
& =\sum_{i \geq 0} F^{n-1}\left(a_{i}\right) x^{(i+1) p^{n-1}-1} d x+\sum_{s=1}^{n-1} \sum_{(j, p)=1} F^{n-1-s}\left(b_{s, j}\right) j x^{j p^{n-1-s}-1} d x .
\end{aligned}
$$

By the induction hypothesis, we have (for all $i, j$, and for $s=1, \ldots, n-2$ )

$$
a_{i}=1+\mathrm{V}^{n-1}\left(e_{i}\right), \quad b_{s, j}=\frac{1}{j}+\mathrm{V}^{n-s-1}\left(f_{s, j}\right),
$$

with $e_{i}, f_{s, j} \in \mathbb{Z}_{(p)}$. Comparing coefficients, we obtain in $\mathbb{Z}_{(p)}$

$$
1=F^{n-1}\left(a_{i}\right)=1+p^{n-1} e_{i}
$$

and for $s=1, \ldots, n-2$,

$$
\frac{1}{j}=F^{n-s-1}\left(b_{s, j}\right)=\frac{1}{j}+p^{n-s-1} f_{s, j} ;
$$

hence $e_{i}=f_{s, j}=0$. Further, we find $b_{n-1, j}=1 / j \in W_{1}\left(\mathbb{Z}_{(p)}\right)$.

7.14. Let $K$ be a field and $\operatorname{Res}_{t}: W_{n} \Omega_{K((t))}^{*} \rightarrow W_{n} \Omega_{K((t))}^{*-1}$ be the residue map (7.9.1). Then for all $r, s \geq 0, i, j \in \mathbb{Z}, a \in W_{n-r}(K)$ and $b \in W_{n-s}(K)$, the following equality holds in $W_{n}(K)$ :

$$
\begin{aligned}
& \operatorname{Res}_{t}\left(\mathrm{~V}^{r}\left([a][t]^{i}\right) d \mathrm{~V}^{s}\left([b][t]^{j}\right)\right)= \\
& \qquad \begin{cases}\operatorname{sgn}(j) \operatorname{gcd}(i, j) \mathrm{V}^{r+s-c}\left([a]^{p^{s-c}}[b]^{p^{r-c}}\right) & \text { if } j p^{r}+i p^{s}=0, \\
0 & \text { otherwise, }\end{cases}
\end{aligned}
$$

where $\operatorname{sgn}(j):=j /|j|$ if $j \neq 0$, and $\operatorname{sgn}(0):=0$ and $c=\min \{r, s\}$ [43, Proposition 2.12].

Lemma 7.15. Set $L \in \Phi$ and let $\sigma: K \hookrightarrow \mathcal{O}_{L}$ be a coefficient field. Let $t \in \mathcal{O}_{L}$ be a local parameter, and set $c \in K$.

(1) Let $r \geq 1$ and write $r=p^{e} r_{0}$, with $\left(r_{0}, p\right)=1, e \geq 0$. Then

$$
\left([t]^{-r_{0}}, 1-t^{r} c\right)_{L, \sigma}=-r_{0} \mathrm{~V}^{e}([c]), \quad \text { in } W_{e+1}(K) .
$$

(2) Let $r \geq 1$ with $(r, p)=1$ and $m=p^{u} m_{0}$, with $\left(m_{0}, p\right)=1, u \geq 1$. Assume $r>m_{0}$. Then for all $n \geq 1$,

$$
\left([t]^{-m}, 1-t^{r} c\right)_{L, \sigma}=0, \quad \text { in } W_{n}(K)
$$


Proof. (1). By Lemmas 7.11 and 7.13, we have

$$
\begin{aligned}
\left([t]^{-r_{0}}, 1-t^{r} c\right)_{L, \sigma}= & \operatorname{Res}_{t}\left([t]^{-r_{0}} \mathrm{~d} \log \left[1-t^{r} c\right]\right) \\
= & -\sum_{i \geq 0} \operatorname{Res}_{t}\left([c]^{i}[t]^{i r-r_{0}} d\left[t^{r} c\right]\right) \\
& -\sum_{s=1}^{e} \sum_{(j, p)=1} \frac{1}{j} \operatorname{Res}_{t}\left([t]^{-r_{0}} d \mathrm{~V}^{s}\left([c]^{j}[t]^{j r}\right)\right) .
\end{aligned}
$$

Now the claim follows from Section 7.14. The proof of (2) is similar.

Lemma 7.16. Set $L \in \Phi$ and let $t \in \mathcal{O}_{L}$ be a local parameter. Let $K \hookrightarrow \mathcal{O}_{L}$ be a coefficient field. Then for $r \geq 1$, any element $a \in \mathrm{fil}_{r}^{\log } W_{n}(L) / W_{n}\left(\mathcal{O}_{L}\right)$ can be written uniquely as

$$
a=\sum_{0>i p^{n-1} \geq-r} a_{i}[t]^{i}+\sum_{s=1}^{n-1} \sum_{\substack{0>j p^{n-1-s} \geq-r \\(j, p)=1}} \mathrm{~V}^{s}\left(b_{s, j}[t]^{j}\right),
$$

where $a_{i} \in W_{n}(K)$ and $b_{s, j} \in W_{n-s}(K)$.

Proof. We can assume $L$ is complete, and hence we have $L=K((t))$. By [21, Lemma 4.1.1] (see also [43, Lemma 2.9]), we can write any element $a$ in $W_{n}(K((t))) / W_{n}(K[[t]])$ uniquely in the form

$$
a=\sum_{0>i} a_{i}[t]^{i}+\sum_{s=1}^{n-1} \sum_{\substack{0>j \\(j, p)=1}} \mathrm{~V}^{s}\left(b_{s, j}[t]^{j}\right)
$$

with $a_{i} \in W_{n}(K)$ and $b_{s, j} \in W_{n-s}(K)$. Now, $a \in \mathrm{fil}_{r}^{\log } W_{n}(L) / W_{n}\left(\mathcal{O}_{L}\right)$ is equivalent to the following equality in $W_{n}(K((t))) / W_{n}(K[[t]])$ :

$$
0=[t]^{r} F^{n-1}(a)=\sum_{0>i} F^{n-1}\left(a_{i}\right)[t]^{i p^{n-1}+r}+\sum_{s=1}^{n-1} \sum_{\substack{0>j \\(j, p)=1}} \mathrm{~V}^{s}\left(F^{n-1}\left(b_{s, j}\right)\right) \cdot[t]^{j p^{n-1-s}+r} .
$$

This yields the statement.

Corollary 7.17. Let $r=p^{e} r_{0} \geq 1$ with $e \geq 0$ and $\left(r_{0}, p\right)=1$. Let $L \in \Phi$ have local parameter $t \in \mathcal{O}_{L}$ and let $\sigma: K \hookrightarrow \mathcal{O}_{L}$ be a coefficient field. Set $\operatorname{gr}_{r}^{\log } W_{n}(L):=$ $\mathrm{fil}_{r}^{\log } W_{n}(L) / \mathrm{fil}_{r-1}^{\log } W_{n}(L), n \geq 1$.

(1) Assume $e \in[0, n-1]$. Then there is a group isomorphism

$$
W_{e+1}(K) \stackrel{\simeq}{\rightarrow} \operatorname{gr}_{r}^{\log } W_{n}(L), \quad b \mapsto \mathrm{V}^{n-1-e}\left(b[t]^{-r_{0}}\right) \bmod \operatorname{fil}_{r-1}^{\log } W_{n}(L) .
$$

(2) Assume $e \geq n$. Then there is a group isomorphism

$$
W_{n}(K) \stackrel{\simeq}{\longrightarrow} \operatorname{gr}_{r}^{\log } W_{n}(L), \quad b \mapsto b[t]^{-p^{e-n+1} r_{0}} \bmod \operatorname{fil}_{r-1}^{\log } W_{n}(L) .
$$


Proof. This follows directly from Lemma 7.16.

Corollary 7.18. Let $r=p^{e} r_{0} \geq 1$ with $e \geq 0$ and $\left(r_{0}, p\right)=1$. Let $L \in \Phi$ have local parameter $t \in \mathcal{O}_{L}$ and let $\sigma: K \hookrightarrow \mathcal{O}_{L}$ be a coefficient field. Set $\operatorname{gr}_{r} W_{n}(L):=\operatorname{fil}_{r} W_{n}(L) / \operatorname{fil}_{r-1} W_{n}(L)$, $n \geq 1$.

(1) Assume $e=0$. Write $r-1=p^{e_{1}} r_{1}$ with $e_{1} \geq 0$ and $\left(r_{1}, p\right)=1$. Then $\operatorname{gr}_{r} W_{n}(L)=0$ if $e_{1} \geq n$, and if $e_{1} \in[0, n-1]$, then there is a group isomorphism

$$
K \stackrel{\simeq}{\rightarrow} \operatorname{gr}_{r} W_{n}(L), \quad b \mapsto \mathrm{V}^{n-1-e_{1}}\left(\left[b t^{-r_{1}}\right]\right) \bmod \operatorname{fil}_{r-1} W_{n}(L) .
$$

(2) Assume $e \in[1, n-1]$. Then there is a group isomorphism

$$
\begin{gathered}
K \oplus W_{e}(K) \stackrel{\simeq}{\rightarrow} \operatorname{gr}_{r} W_{n}(L), \\
(b, c) \mapsto \mathrm{V}^{n-1}\left(b t^{-(r-1)}\right)+\mathrm{V}^{n-e}\left(c[t]^{-r_{0} p}\right) \bmod \operatorname{fil}_{r-1} W_{n}(L) .
\end{gathered}
$$

(3) Assume $e \geq n$. Then there is a group isomorphism

$$
\begin{gathered}
K \oplus W_{n}(K) \stackrel{\simeq}{\rightarrow} \operatorname{gr}_{r} W_{n}(L), \\
(b, c) \mapsto \mathrm{V}^{n-1}\left(b t^{-(r-1)}\right)+c[t]^{-p^{e-n+1} r_{0}} \bmod \operatorname{fil}_{r-1} W_{n}(L) .
\end{gathered}
$$

Proof. Define $e^{\prime}:=\min \{e, n\}$ and recall

$$
\mathrm{fil}_{r} W_{n}(L)=\mathrm{fil}_{r-1}^{\log } W_{n}(L)+\mathrm{V}^{n-e^{\prime}} \mathrm{fil}_{r}^{\log } W_{e^{\prime}}(L) .
$$

Thus (2) and (3) follow directly from Lemma 7.16. (For the injectivity in (2), we use the fact that $\mathrm{V}^{n-e}\left(c[t]^{-r_{0} p}\right)=\mathrm{V}^{n-e-1}\left(\mathrm{~V}(c)[t]^{-r_{0}}\right)$.) Furthermore, it is immediate from Lemma 7.16 that there is an injective map as in (1) and that any element in the target has a representative of the form $\mathrm{V}^{n-1-e_{1}}\left(\beta[t]^{-r_{1}}\right)$ with $\beta \in W_{e_{1}}(K)$. Thus the statement follows if we show $\mathrm{V}^{n-1-e_{1}}\left(\mathrm{~V}\left(\beta_{1}\right)[t]^{-r_{1}}\right) \in \mathrm{fil}_{r-1} W_{n}(L)$. But by Lemma 7.16 the element $\mathrm{V}^{n-1-e_{1}}\left(\mathrm{~V}\left(\beta_{1}\right)[t]^{-r_{1}}\right)=\mathrm{V}^{n-e_{1}}\left(\beta_{1}[t]^{-p r_{1}}\right)$ lies in $\mathrm{V}^{n-e_{1}} \operatorname{fil}_{r-1}^{\log } W_{e_{1}}(L) \subset \operatorname{fil}_{r-1} W_{n}(L)$. Hence the statement.

Proposition 7.19. Let $L \in \Phi$ have residue field $\kappa_{L}$ and local parameter $t \in \mathcal{O}_{L}$. Let $z_{1}, \ldots, z_{m} \subset \mathcal{O}_{L}$ be a lift of some $p$-basis of $\kappa / k$. Let $\sigma_{0}: K_{0} \hookrightarrow \mathcal{O}_{L}$ be the unique coefficient field with $z_{i} \in K_{0}, i=1, \ldots, m$. Let $x$ be an indeterminate and set $L_{x}:=\operatorname{Frac}\left(\mathcal{O}_{L}[x]_{(t)}^{h}\right)$. Denote also by $\sigma_{0}: K_{0}(x) \hookrightarrow L_{x}$ the canonical extension of $\sigma_{0}$. Let $r \geq 1$ and set $a \in$ $\mathrm{fil}_{r}^{F} W_{n}(L)$. Assume one of the following:

(1) $(r, p)=1$ or $r=p=2$ or $m=0$, and $\left(a, 1-x t^{r-1}\right)_{L_{x}, \sigma_{0}}=0$.

(2) $r>2, p \mid r, m \geq 1$ and $\left(a, 1-x t^{r-1}\right)_{L_{x}, \sigma_{j}}=0$, for $j=0,1$, where $\sigma_{1}: K_{1} \hookrightarrow \mathcal{O}_{L}$ is the unique coefficient field with $z_{i} /\left(1+z_{i}^{p^{e}} t\right) \in K_{1}$ for all $i$, with $e=\operatorname{ord}_{p}(r)$, and we denote also by $\sigma_{1}: K_{1}(x) \hookrightarrow \mathcal{O}_{L_{x}}$ the canonical extension.

Then $a \in \operatorname{fil}_{r-1}^{F} W_{n}(L)$. 
Proof. Since $k$ is perfect, a $p$-basis over $k$ is the same as a separating transcendence basis over $k$ (see, for example, [18, Théorème 0.21 .4 .5$]$ ), hence there are unique coefficient fields $K_{0}$ and $K_{1}$ as in the statement [4, IX, §3, No. 2]. By Proposition 7.5 and Corollary 4.24, we know fil ${ }_{r-1}^{F} W_{n}(L) \subset W_{n}\left(\mathcal{O}_{L}, \mathfrak{m}_{L}^{-r+1}\right)$; furthermore, for all $b \in W_{n}\left(\mathcal{O}_{L}, \mathfrak{m}_{L}^{-r+1}\right)$ we have $\left(b, 1-x t^{r-1}\right)_{L_{x}, \sigma}=0$ for all coefficient fields $\sigma$ (by Corollary 4.40). Thus in the following we may replace $a$ by $a+b$ with $b \in$ fil $_{r-1}^{F} W_{n}(L)$. We will use $\sigma_{0}$ to identify $\hat{L}=K_{0}((t))$.

Write $r=p^{e} r_{0}$ with $e \geq 0$ and $\left(r_{0}, p\right)=1$. We distinguish four cases.

Case 1: $e=0$. Write $r-1=p^{e_{1}} r_{1}$ with $\left(r_{1}, p\right)=1$ and $e_{1} \geq 0$. By Corollary 7.18(1) we have $\operatorname{gr}_{r} W_{n}(L)=0$ if $e_{1} \geq n$, and there is nothing to show; if $e_{1} \in[0, n-1]$, we have

$$
a \equiv \sum_{h \geq 0} F^{h} \mathrm{~V}^{n-1-e_{1}}\left(\left[b_{h}\right][t]^{-r_{1}}\right) \bmod \operatorname{fil}_{r-1}^{F} W_{n}(L),
$$

with $b_{h} \in K_{0}$. We compute in $W_{n}\left(K_{0}(x)\right)$

$$
\begin{array}{rlrl}
0 & =\left(\sum_{h} F^{h}\left(\mathrm{~V}^{n-1-e_{1}}\left(\left[b_{h}\right][t]^{-r_{1}}\right)\right), 1-x t^{r-1}\right)_{L_{x}, \sigma_{0}}, & & \text { by }(1), \\
& =\sum_{h} F^{h} \mathrm{~V}^{n-1-e_{1}}\left(\left[b_{h}\right] \cdot\left([t]^{-r_{1}}, 1-x t^{r-1}\right)_{L_{x}, \sigma_{0}}\right), & & \text { by Lemma } 7.11, \\
& =-r_{1} \sum_{h} F^{h} \mathrm{~V}^{n-1-e_{1}}\left(\left[b_{h}\right] \mathrm{V}^{e_{1}}([x])\right), & & \text { by Lemma } 7.15(1), \\
& =-r_{1} V^{n-1}\left(\sum_{h} b_{h}^{p^{e_{1}+h}} x^{p^{h}}\right) . &
\end{array}
$$

Hence $b_{h}=0$ for all $h \geq 0$, which completes the proof of the first case.

Case 2: $r=p=2$. By Corollary 7.18(2) and (3) we have

$$
a \equiv \sum_{h} F^{h} \mathrm{~V}^{n-1}\left(b_{h} t^{-1}+c_{h} t^{-2}\right) \bmod W_{n}\left(\mathcal{O}_{L}\right),
$$

with $b_{h}, c_{h} \in K_{0}$. Note that

$$
\operatorname{Res}_{t}\left(t^{-1} \operatorname{dlog}(1-x t)\right)=x, \quad \operatorname{Res}_{t}\left(t^{-2} \operatorname{dlog}(1-x t)\right)=x^{2} .
$$

Hence by (1),

$$
0=(a, 1-x t)_{L_{x}, \sigma_{0}}=\mathrm{V}^{n-1}\left(\sum_{h} b_{h}^{2^{h}} x^{2^{h}}+c_{h}^{2^{h}} x^{2^{h+1}}\right)
$$

We obtain

$$
b_{0}=0 \quad \text { and } \quad c_{h}=b_{h+1}^{2}, \quad \text { all } h \geq 0 .
$$

Thus reshuffling the sum defining $a$, we obtain

$$
a=\sum_{h} F^{h} \mathrm{~V}^{n-1}\left(b_{h} t^{-1}+F\left(b_{h+1} t^{-1}\right)\right)=2 \sum_{h} F^{h} \mathrm{~V}^{n-1}\left(b_{h} t^{-1}\right)=0 .
$$


Case 3: $1 \leq e \leq n-1$ and $r>2$. By Corollary 7.18(2) we have

$$
a \equiv \sum_{h \geq 0} F^{h}\left(\mathrm{~V}^{n-1}\left(b_{h}[t]^{-(r-1)}\right)+\mathrm{V}^{n-e}\left(c_{h}[t]^{-r_{0} p}\right)\right) \bmod \operatorname{fil}_{r-1}^{F} W_{n}(L),
$$

where $b_{h} \in K_{0}$ and $c_{h} \in W_{e}\left(K_{0}\right)$. By a similar computation as in the first case, the vanishing $\left(a, 1-x t^{r-1}\right)_{L_{x}, \sigma_{0}}=0$ together with $r-1>r_{0}$ and Lemma 7.15(1) and (2) imply $b_{h}=0$, for all $h \geq 0$. Thus

$$
a \equiv \sum_{h \geq 0} F^{h}\left(\mathrm{~V}^{n-e}\left(c_{h}[t]^{-r_{0} p}\right)\right) \bmod \operatorname{fil}_{r-1}^{F} W_{n}(L) .
$$

It suffices to show

$$
c_{h} \in F W_{e}\left(K_{0}\right), \quad \text { all } h \geq 0 .
$$

Indeed, then $V^{n-e}\left(c_{h}[t]^{-r_{0} p}\right)=F V^{n-e}\left(c_{h}^{\prime}[t]^{-r_{0}}\right)$, for some $c_{h}^{\prime} \in W_{e}\left(K_{0}\right)$, which lies in $F$ fil $_{r / p}^{\log } W_{n}(L) \subset F$ fil $_{r-2}^{\log } W_{n}(L)$ (use $r \geq 3$ for the last inclusion).

If $m=\operatorname{trdeg}(\kappa / k)=0$, then $K_{0}$ is perfect and formula (7.19.1) holds. This completes the proof of the implication: $(1) \Rightarrow a \in \mathrm{fil}_{r-1} W_{n} \mathcal{O}_{L}$.

Now assume $m \geq 1$. We prove formula (7.19.1) by contradiction using $\left(a, 1-x t^{r-1}\right)_{L_{x}, \sigma_{1}}$ $=0$ with $\sigma_{1}: K_{1}(x) \hookrightarrow \mathcal{O}_{L_{x}}$ as in (2). Thus, assume that not all $c_{h}$ are in $F W_{e}\left(K_{0}\right)$. Let $h_{0}$ be the minimal $h$ with $c_{h} \notin F W_{e}\left(K_{0}\right)$. Hence, modulo fil ${ }_{r-1}^{F} W_{n}(L)$, we can write $a$ as $F^{h_{0}}\left(V^{n-e}\left(a^{\prime}\right)\right)$, with $a^{\prime}=\sum_{h \geq h_{0}} F^{h-h_{0}}\left(c_{h}\left[t^{-r_{0} p}\right]\right)$. Since $F: W_{n}\left(K_{j}(x)\right) \rightarrow W_{n}\left(K_{j}(x)\right)$ and $V^{n-e}: W_{e}\left(K_{j}(x)\right) \rightarrow W_{n}\left(K_{j}(x)\right), j=0,1$, are injective, the element $a^{\prime}$ also satisfies $\left(a^{\prime}, 1-x t^{r-1}\right)_{L_{x}, \sigma_{j}}=0, j=0,1$. Thus we can assume $n=e$ and $h_{0}=0-$ that is, $c_{0} \notin$ $F W_{e}\left(K_{0}\right)$ - and we want to find a contradiction. Since the elements $z_{1}, \ldots, z_{m} \in K_{0}$ from the statement form a $p$-basis, we can write $c_{0}$ as

$$
c_{0}=\sum_{j=0}^{e-1} V^{j}\left(\sum_{I \subset[0, p-1]^{m}}\left[a_{I, j}\right]^{p}[z]^{I}\right)
$$

where $a_{I, j} \in K_{0}$ and $[z]^{I}=\left[z_{1}\right]^{i_{1}} \cdots\left[z_{m}\right]^{i_{m}}$, for $I=\left(i_{1}, \ldots, i_{m}\right)$. Therefore, $c_{0} \notin F W_{e}\left(K_{0}\right)$ translates into

$$
\exists j \in[0, e-1], I \in[0, p-1]^{m} \backslash\{(0, \ldots, 0)\} \text { such that } \quad a_{I, j} \neq 0 .
$$

Since we want to compute the local symbol with respect to the coefficient field $\sigma_{1}$ : $K_{1}(x) \hookrightarrow \mathcal{O}_{L_{x}}$, we have to rewrite $c_{0}$ as an element in $W_{n}\left(K_{1}[[t]]\right)$. Define

$$
y_{i}:=\frac{z_{i}}{1+z_{i}^{p^{e}} t} \in K_{1}, \quad i=1, \ldots, m .
$$

Then

$$
c_{0}=\sum_{j=0}^{e-1} V^{j}\left(\sum_{I \subset[0, p-1]^{m}}\left[a_{I, j}\right]^{p}\left[y\left(1+z^{p^{e}} t\right)\right]^{I}\right)
$$


where $\left[y\left(1+z^{p^{e}} t\right)\right]^{I}:=\prod_{h=1}^{m}\left[y_{h}\left(1+z_{h}^{p^{e}} t\right)\right]^{i_{h}}$. Note that $a_{I, j}, z_{h} \in K_{0} \subset K_{1}[[t]]$ are not constant. The composition $\mathrm{F}^{e^{-1}} d: W_{e}(-) \rightarrow \Omega^{1}$ is a morphism of reciprocity sheaves (see Lemma 7.7). Hence $\mathrm{F}^{e-1} d$ commutes with the local symbol, which on $\Omega^{1}$ is given by $(\alpha, f)_{L_{x}, \sigma_{1}}=\operatorname{Res}_{K_{1}((t))}(\alpha \wedge \operatorname{dlog} f)$ (see Lemma 7.11). Using $\mathrm{F}^{e-1} d F=0$ on $W_{e}$, we obtain the following equalities in $\Omega_{K_{1}(x)}^{1}$ :

$$
\begin{aligned}
0 & =\mathrm{F}^{e-1} d\left(a, 1-x t^{r-1}\right)_{L_{x}, \sigma_{1}} \\
& =\left(\mathrm{F}^{e-1} d\left(c_{0}[t]^{-r_{0} p}\right), 1-x t^{r-1}\right)_{L_{x}, \sigma_{1}} \\
& =\sum_{j=0}^{e-1} \sum_{I}\left(\mathrm{~F}^{e-1-j} d\left(\left[a_{I, j}\right]^{p}\left[y\left(1+z^{p^{e}} t\right)\right]^{I}[t]^{-r_{0} p^{j+1}}\right), 1-x t^{r-1}\right)_{L_{x}, \sigma_{1}} \\
& =\sum_{j=0}^{e-1} \sum_{I} \operatorname{Res}_{t}\left(a_{I, j}^{p^{e-j}} t^{-r} \mathrm{~F}^{e-1-j} d\left(\left[y\left(1+z^{p^{e}} t\right)\right]^{I}\right) \operatorname{dlog}\left(1-x t^{r-1}\right)\right) .
\end{aligned}
$$

Write

$$
a_{I, j}=\bar{a}_{I, j}+t b_{I, j}, \quad \bar{a}_{I, j} \in K_{1}, b_{I, j} \in K_{1}[[t]]
$$

Denote by

$$
\bar{\sigma}_{j}: K_{j} \stackrel{\simeq}{\rightarrow} \kappa_{L}, \quad j=0,1,
$$

the isomorphisms induced by $\sigma_{j}: K_{j} \hookrightarrow \mathcal{O}_{L}$. Then $\bar{\sigma}_{1}\left(\bar{a}_{I, j}\right)=\bar{\sigma}_{0}\left(a_{I, j}\right)$; in particular,

$$
a_{I, j}=0 \Longleftrightarrow \bar{a}_{I, j}=0 \text {. }
$$

For $j \in[0, e-1]$, we have $a_{I, j}^{p^{e-j}} \equiv \bar{a}_{I, j}^{p^{e-j}} \bmod t^{2}$, and thus we obtain from the previous computation

$$
0=-\sum_{j=0}^{e-1} \sum_{I \subset[0, p-1]^{m}} \operatorname{Res}_{t}\left(\bar{a}_{I, j}^{p^{e-j}} t^{-r} \mathrm{~F}^{e-1-j} d\left(\left[y\left(1+z^{p^{e}} t\right)\right]^{I}\right) d\left(x t^{r-1}\right)\right) .
$$

We have

$$
\mathrm{F}^{e-1-j} d\left[y\left(1+z^{p^{e}} t\right)\right]^{I}=\sum_{h=1}^{m} i_{h}\left(y\left(1+z^{p^{e}} t\right)\right)^{I p^{e-1-j}} \mathrm{~d} \log \left(y_{h}\left(1+z_{h}^{p^{e}} t\right)\right) .
$$

Note that

$$
z_{h}=y_{h}+t \zeta_{h}, \quad \zeta_{h} \in K_{1}[[t]], h=1, \ldots, m .
$$

Thus, $z_{h}^{p^{e}} \equiv y_{h}^{p^{e}} \bmod t^{p^{e}}$. Hence the coefficient of $\mathrm{F}^{e-1-j} d\left[y\left(1+z^{p^{e}} t\right)\right]^{I}$ in $K_{1}$ in front of $d t$ is equal to

$$
f_{I, j}:=q_{I}^{p^{e}} y^{I p^{e-1-j}}, \text { with } q_{I}=\sum_{h=1}^{m} i_{h} y_{h}
$$


the coefficient of $\mathrm{F}^{e-1-j} d\left[y\left(1+z^{p^{e}} t\right)\right]^{I}$ in $\Omega_{K_{1}}^{1}$ in front of $t$ is equal to $d f_{I, j}$. (This is 0 if $j \in[0, e-2]$.) Thus by equation (7.19.4) we have

$$
0=\sum_{j=0}^{e-1} \sum_{I}\left(\bar{a}_{I, j}^{p^{e-j}}\left(f_{I, j} d x+x d f_{I, j}\right)\right)=d\left(\sum_{j=0}^{e-1} \sum_{I} \bar{a}_{I, j}^{p^{e-j}} f_{I, j} \cdot x\right) .
$$

Hence the element in the brackets has to be a $p$ th power - that is, by definition (7.19.6),

$$
K_{1}^{p} \ni \sum_{j=0}^{e-2}\left(\sum_{I \subset[0, p-1]^{m}}\left(\bar{a}_{I, j} q_{I}^{p^{j}}\right)^{p} y^{I}\right)^{p^{e-1-j}} \cdot x+\sum_{\substack{I \subset[0, p-1]^{m} \\ I \neq 0}}\left(\bar{a}_{I, e-1} q_{I}^{p^{e-1}}\right)^{p} y^{I} x .
$$

Note that

$$
q_{I}=0 \Longleftrightarrow I=0
$$

Since $y_{1}, \ldots, y_{m}$ and $x$ form a $p$-basis of $K_{1}(x)$ over $k$, we obtain

$$
\bar{a}_{I, e-1}=0, \quad \text { for all } I \subset[0, p-1]^{m} \backslash\{(0, \ldots, 0)\},
$$

and

$$
\sum_{j=0}^{e-2} \sum_{I \subset[0, p-1]^{m}}\left(\bar{a}_{I, j} q_{I}^{p^{j}}\right)^{p^{e-1-j}} y^{I p^{e-2-j}}=0 .
$$

Since $y_{1}, \ldots, y_{m} \in K_{1}$ form a $p$-basis over $k$, we obtain, similarly as before, $\bar{a}_{I, e-2}=0$ for all $I \neq 0$. We may proceed in this way and obtain

$$
\bar{a}_{I, j}=0, \quad \text { for all } I \neq 0, j \geq 0 .
$$

By formula (7.19.3), this contradicts formula (7.19.2) and proves the statement in this case.

Case 4: $e \geq n$ and $r>2$. By Corollary 7.18(3), we have

$$
a \equiv \sum_{h \geq 0} F^{h}\left(c_{h}[t]^{-p^{e-n+1} r_{0}}+V^{n-1}\left(b_{h}[t]^{-(r-1)}\right)\right) \bmod \operatorname{fil}_{r-1}^{F} W_{n}(L),
$$

where $c_{h} \in W_{n}\left(K_{0}\right)$ and $b_{h} \in K_{0}$. As before, it follows from $\left(a, 1-x t^{r-1}\right)_{L_{x}, \sigma_{0}}=0$ and Lemma 7.15 that $b_{h}=0$ for all $h \geq 0$. Thus

$$
a \equiv \sum_{h \geq 0} F^{h}\left(c_{h}[t]^{-p^{e-n+1} r_{0}}\right) \bmod \operatorname{fil}_{r-1}^{F} W_{n}(L) .
$$

Applying $\mathrm{V}^{e-n+1}$, we obtain

$$
\mathrm{V}^{e-n+1}(a) \equiv \sum_{h \geq 0} F^{h} \mathrm{~V}\left(c_{h}^{\prime}[t]^{-r_{0} p}\right) \bmod \operatorname{fil}_{r-1}^{F} W_{e+1}(L),
$$

where $c_{h}^{\prime}=V^{e-n}\left(c_{h}\right) \in W_{e}\left(K_{0}\right)$. Since $V^{e-n+1}(a) \in \mathrm{fil}_{r} W_{e+1}(L)$, we can apply the third case, in particular formula (7.19.1), to conclude $c_{h} \in F W_{n}\left(K_{0}\right)$, and then also $a \in \operatorname{fil}_{r-1}^{F} W_{n}(L)$. This completes the proof of the proposition. 
Theorem 7.20. Set $L \in \Phi$ and $r \geq 0$. Then

$$
\operatorname{fil}_{r}^{F} W_{n}(L)=\widetilde{W}_{n}\left(\mathcal{O}_{L}, \mathfrak{m}^{-r}\right)
$$

that is, the Brylinski-Kato-Matsuda-Russell conductor is motivic.

Proof. We have $\operatorname{fil}_{r}^{F} W_{n}(L) \subset \widetilde{W}_{n}\left(\mathcal{O}_{L}, \mathfrak{m}^{-r}\right)$, by Proposition 7.5 and Theorem $4.15(4)$, and we know this is an equality for $r=0$. Let $t \in \mathcal{O}_{L}$ be a local parameter. By Corollary 4.40, we have

$$
a \in \widetilde{W}_{n}\left(\mathcal{O}_{L}, \mathfrak{m}^{-r}\right) \Rightarrow\left(a, 1-x t^{m}\right)_{L_{x}, \sigma}=0, \quad \text { for all } m \geq r \text { and all } \sigma
$$

where $L_{x}=\operatorname{Frac}\left(\mathcal{O}_{L}[x]_{(t)}^{h}\right)$ and $\sigma$ runs through all coefficient fields $\sigma: K \hookrightarrow \mathcal{O}_{L}$. Furthermore, we know that for any $a \in \widetilde{W}_{n}\left(\mathcal{O}_{L}, \mathfrak{m}^{-r}\right)$ there exists some $m \geq r$ such that $a \in \mathrm{fil}_{m}^{F} W_{n}(L)$. Hence the statement follows from Proposition 7.19.

\section{Lisse sheaves of rank 1 and the Artin conductor}

In this section $k$ is a perfect field of characteristic $p>0$.

\subsection{The case of finite monodromy}

8.1. Consider the constant presheaf with transfers $\mathbb{Q} / \mathbb{Z}$ - that is, an elementary correspondence $V \in \operatorname{Cor}(X, Y)$, with $X, Y$ smooth and connected, that acts by multiplication with $[V: X]$. By [39, Lemma 6.23],

$$
X \mapsto H^{1}(X):=H_{\text {ét }}^{1}(X, \mathbb{Q} / \mathbb{Z})=\operatorname{Hom}_{\text {cont }}\left(\pi_{1}(X)^{\mathrm{ab}}, \mathbb{Q} / \mathbb{Z}\right)
$$

is a presheaf with transfers, which we denote by $H^{1}$ in the following.

Note that $H^{1} \in \mathbf{N S T}$, as follows from the following lemma:

Lemma 8.2. Let $A$ be an abelian group. It defines a constant étale sheaf on $\mathbf{S m}$. Then the presheaf $X \mapsto H_{\text {ét }}^{1}(X, A)$ is a Nisnevich sheaf on $\mathbf{S m}$.

Proof. Let $\mathcal{H}^{i}$ be the Nisnevich sheafification of $X \mapsto H_{\text {ét }}^{i}(X, A)$. Then for any $X \in \mathbf{S m}$, we have an exact sequence

$$
H_{\mathrm{Nis}}^{1}\left(X, \mathcal{H}^{0}\right) \rightarrow H_{\text {ét }}^{1}(X, A) \rightarrow H_{\mathrm{Nis}}^{0}\left(X, \mathcal{H}^{1}\right) \rightarrow H_{\mathrm{Nis}}^{2}\left(X, \mathcal{H}^{0}\right) .
$$

But $\mathcal{H}^{0}=A$ is constant, and hence by [49, Theorem 3.1.12] we have $H_{\mathrm{Nis}}^{i}\left(X, \mathcal{H}^{0}\right)=$ $H_{\text {Zar }}^{i}\left(X, \mathcal{H}^{0}\right)=0$ for all $i \geq 1$. Thus the presheaf from the statement is equal to $\mathcal{H}^{1}$.

Lemma 8.3. The Artin-Schreier-Witt sequence

$$
0 \rightarrow \mathbb{Z} / p^{n} \mathbb{Z} \rightarrow W_{n} \stackrel{F-1}{\longrightarrow} W_{n} \rightarrow 0
$$

is an exact sequence of étale sheaves with transfers on $\mathbf{S m}$, where $F: W_{n} \rightarrow W_{n}$ is the base change over Speck of the Frobenius on the $\mathbf{F}_{p}$-group scheme $W_{n}$. 
Proof. The exactness of sequence (8.3.1) on $X_{\text {ét }}$ is classical. The map $F-1: W_{n} \rightarrow W_{n}$ is a morphism of $k$-group schemes, and hence is compatible with transfers; for the inclusion $\mathbb{Z} / p^{n} \mathbb{Z} \hookrightarrow W_{n}$, this follows directly from Lemma 7.2.

8.4. We denote by $\delta_{n}$ the composition

$$
\delta_{n}: W_{n}(L) \rightarrow W_{n}(L) /(F-1) W_{n}(L) \cong H_{\text {ét }}^{1}\left(L, \mathbb{Z} / p^{n} \mathbb{Z}\right):=H_{p^{n}}^{1}(L),
$$

which is the connecting homomorphism stemming from the Artin-Schreier-Witt sequence (8.3.1). Then we set

$$
\mathrm{fil}_{j} H_{p^{n}}^{1}(L):=\delta_{n}\left(\operatorname{fil}_{j} W_{n}(L)\right)=\delta_{n}\left(\operatorname{fil}_{j}^{F} W_{n}(L)\right) .
$$

For $j \geq 0$, we set

$$
\mathrm{fil}_{j} H^{1}(L):= \begin{cases}\operatorname{Im}\left(H^{1}\left(\mathcal{O}_{L}\right) \rightarrow H^{1}(L)\right) & \text { if } j=0 \\ H^{1}(L)\left\{p^{\prime}\right\} \oplus \bigcup_{n \geq 1} \text { fil }_{j} H_{p^{n}}^{1}(L) & \text { if } j \geq 1\end{cases}
$$

with $H^{1}(L)\left\{p^{\prime}\right\}=\bigoplus_{\ell \neq p} H_{\text {ét }}^{1}\left(L, \mathbb{Q}_{\ell} / \mathbb{Z}_{\ell}\right)$ the prime-to- $p$ part of $H^{1}(L)$.

For $\chi \in H^{1}(L)$, we define

$$
\operatorname{Art}_{L}(\chi)=\min \left\{j \geq 0 \mid \chi \in \operatorname{fil}_{j} H^{1}(L)\right\} .
$$

Proposition 8.5. The collection

$$
\operatorname{Art}=\left\{\operatorname{Art}_{L}: H^{1}(L) \rightarrow \mathbb{N}_{0} \mid L \in \Phi\right\}
$$

is a semicontinuous conductor on $H^{1}$, as is its restriction $\mathrm{Art} \leq 1$.

Proof. By Proposition 7.5 and Lemma 4.28, Art satisfies (c1)-(c6) except possibly for (c4). (For (c5), note that $W_{n}(Y) \rightarrow H_{p^{n}}^{1}(Y)$ is surjective for any affine scheme over $k$.) It remains to show that $\mathrm{Art}^{\leq 1}$ satisfies (c4). Let $X$ be a smooth $k$-scheme and set $a \in H^{1}\left(\mathbf{A}_{X}^{1}\right)$ with

$$
\operatorname{Art}_{k(x)_{\infty}}^{1}\left(\rho_{x}^{*} a\right) \leq 1, \quad \text { for all closed points } x \in X_{(0)},
$$

where $\rho_{x}: \operatorname{Spec} k(x)(t)_{\infty}=\operatorname{SpecFrac}\left(\mathcal{O}_{\mathbf{P}_{x}^{1}, \infty}^{h}\right) \rightarrow \mathbf{A}_{X}^{1}$ is the natural map. We want to show : $a \in H^{1}(X)$. Since $H^{1}=H^{1}\left\{p^{\prime}\right\} \oplus \lim _{\rightarrow} H_{p^{n}}^{1}$, with $H^{1}\left\{p^{\prime}\right\}$ the $\mathbf{A}^{1}$-invariant subsheaf of prime-to- $p$ torsion, we can assume $a \in H_{p^{n}}^{1}\left(\mathbf{A}_{X}^{1}\right)$. Furthermore, the question is local on $X$, so we can assume $X=\operatorname{Spec} A$ affine. We consider first the case where $n=1$. Condition (8.5.1) implies

$$
\rho_{x}^{*} a \in \operatorname{Im}\left(H_{p}^{1}\left(\mathcal{O}_{\mathbf{P}_{x}^{1}, \infty}^{h}\right) \rightarrow H_{p}^{1}\left(k(x)(t)_{\infty}\right)\right) .
$$

Denote by $a(x)$ the restriction of $a$ to $\mathbf{A}_{x}^{1}$. Since $H_{p}^{1}$ is a Nisnevich sheaf, we conclude

$$
a(x) \in H_{p}^{1}\left(\mathbf{P}_{x}^{1}\right)=H_{p}^{1}(x) .
$$


Thus we find a polynomial $\tilde{a}=a_{0}+a_{1} t+\cdots+a_{n} t^{n} \in A[t]$ mapping to $a$ such that for all closed points $x \in X$, there exist $b_{x} \in k(x)$ and $g_{x} \in k(x)[t]$ with

$$
\tilde{a}(x)=b_{x}+g_{x}^{p}-g_{x}, \quad \text { in } k(x)[t] .
$$

Assume $n \geq 1$. Then $n=p \cdot n_{1}$ for some $n_{1} \geq 1$. We claim

$$
a_{n}=c_{1}^{p}, \quad \text { some } c_{1} \in A^{p} .
$$

Indeed, write $n=p^{e} m$ with $e \geq 1$ and $(p, m)=1$, and for a fixed closed point $x \in X$ write $g_{x}=c_{0}+c_{1} t+\cdots+c_{p^{e-1} m} t^{p^{e-1} m}$; then equation (8.5.3) implies

$$
a_{n}(x)=c_{p^{e-1} m}^{p}, \quad a_{p^{i} m}(x)=c_{p^{i-1} m}^{p}-c_{p^{i} m}, i \in[1, e-1], \quad a_{m}(x)=-c_{m} .
$$

Hence for all maximal ideals $\mathfrak{m} \subset A$, we have

$$
a_{n} \equiv \sum_{j=0}^{e-1}\left(-a_{p^{j} m}\right)^{p^{e-j}} \bmod \mathfrak{m} .
$$

It follows that $a_{n}=\left(\sum_{j=0}^{e-1}\left(-a_{p^{j} m}\right)^{p^{e-j-1}}\right)^{p} \in A^{p}$, which yields equation (8.5.4).

Now $a^{(1)}=\tilde{a}-\left(c_{1} t^{n_{1}}\right)^{p}+c_{1} t^{n_{1}}$ also has property (8.5.3), and its degree is strictly smaller than $n$. We can replace $a$ by $a^{(1)}$ in the foregoing discussion and go on in this way until we reach a polynomial $a^{(r)} \in A[t]$ whose degree is strictly smaller than $p$, in which case equation (8.5.3) forces it to be constant $=c_{r} \in A$. We obtain

$$
\tilde{a}=c_{r}+\sum_{i=1}^{r-1}\left(c_{i} t^{n_{i}}\right)^{p}-c_{i} t^{n_{i}},
$$

whence $a \in H_{p}^{1}(X)$.

Let $n \geq 1$. If $a \in H_{p^{n}}^{1}\left(\mathbf{A}_{X}^{1}\right)$ satisfies formula (8.5.1), then so does $p^{n-1} a \in H_{p}^{1}\left(\mathbf{A}_{X}^{1}\right)$. In the case where $n=1$, by the exact sequence

$$
0 \rightarrow H_{p^{n-1}}^{1}(X) \rightarrow H_{p^{n}}^{1}(X) \stackrel{p^{n-1}}{\longrightarrow} H_{p}^{1}(X) \rightarrow 0
$$

( $X$ is affine) we find an element $b \in H_{p^{n}}^{1}(X)$ such that $p^{n-1}(a-b)=0$. Since $a-b$ also satisfies formula (8.5.1), we obtain $a-b \in H_{p^{n-1}}^{1}(X)$ by induction. This completes the proof.

Lemma 8.6. Let $K$ be a field of positive characteristic, $x$ an indeterminate and $g \in$ $W_{n}(K(x))$. Assume $F(g)-g=\mathrm{V}^{n-1}(b x)$ for some $b \in K$. Then $g \in \mathbb{Z} / p^{n} \mathbb{Z}$ - that is, $F(g)-g=0$.

Proof. If $n=1$, then $g^{p}-g=b x$ forces $g$ to be constant and hence $g^{p}-g=0$ - that is, $g \in$ $\mathbf{F}_{p}$. If $n \geq 2$, then $F(g)-g$ is 0 when restricted to $W_{n-1}(K(x))$. Hence $g=m \cdot[1]+\mathrm{V}^{n-1}(f)$ with $f \in K(x), m \in \mathbb{Z}$. Thus $F(f)-f=b x$, and we conclude with the case where $n=1$. 
Proposition 8.7. Let $L, t \in \mathcal{O}_{L}$ and $\sigma_{j}: K_{j} \hookrightarrow \mathcal{O}_{L}, j=0,1$, be as in Proposition 7.19. We also denote by $\sigma_{j}: K_{j}(x) \hookrightarrow \mathcal{O}_{L_{x}}$ the canonical extension. Set $r \geq 1$ and $a \in \mathrm{fil}_{r} H_{p^{n}}^{1}(L)$. Assume one of the following:

(1) $(r, p)=1$ or $r=p=2$ or $m=0$, and $\left(a, 1-x t^{r-1}\right)_{L_{x}, \sigma_{0}}=0$.

(2) $r>2, p \mid r, m \geq 1$ and $\left(a, 1-x t^{r-1}\right)_{L_{x}, \sigma_{j}}=0$, for $j=0,1$.

Then $a \in$ fil $_{r-1} H_{p^{n}}^{1}(L)$.

Proof. Let $\tilde{a} \in \mathrm{fil}_{r} W_{n}(L)$ be a lift of $a$. If $\left(a, 1-x t^{r-1}\right)_{L_{x}, \sigma_{j}}=0$, for some $j \in\{0,1\}$, then we find $g_{j} \in W_{n}\left(K_{j}(x)\right)$ such that

$$
\left(\tilde{a}, 1-x t^{r-1}\right)_{L_{x}, \sigma_{j}}=F\left(g_{j}\right)-g_{j} .
$$

It suffices to show $\tilde{a} \in \mathrm{fil}_{r-1}^{F} W_{n}(L)$. Write $r=p^{e} r_{0}$ with $e \geq 0$ and $\left(r_{0}, p\right)=1$.

Case 1: $e=0$. Write $r-1=p^{e_{1}} r_{1}$ with $e_{1} \geq 0$ and $\left(p, r_{1}\right)=0$. If $e_{1} \geq n$, then by Corollary 7.18(1) we have $\mathrm{fil}_{r} H_{p^{n}}^{1}(L)=\mathrm{fil}_{r-1} H_{p^{n}}^{1}(L)$; otherwise we have

$$
\tilde{a} \equiv \mathrm{V}^{n-1-e_{1}}\left([b][t]^{-r_{1}}\right) \bmod \mathrm{fil}_{r-1} W_{n}(L)
$$

for some $b \in K_{0}$. Thus

$$
\begin{aligned}
F\left(g_{0}\right)-g_{0} & =\left(\tilde{a}, 1-x t^{r-1}\right)_{L_{x}, \sigma_{0}}, & & \text { by }(1), \\
& =\mathrm{V}^{n-1-e_{1}}\left([b]\left([t]^{-r_{1}}, 1-x t^{r-1}\right)_{L_{x}, \sigma_{0}}\right), & & \text { by Lemma } 7.11, \\
& =-r_{1} \mathrm{~V}^{n-1}\left(b^{p^{e_{1}}} x\right), & & \text { by Lemma } 7.15(1) .
\end{aligned}
$$

Lemma 8.6 implies $F\left(g_{0}\right)-g_{0}=0$. Hence $\tilde{a} \in \mathrm{fil}_{r-1}^{F} W_{n}(L)$, by Proposition 7.19(1).

Case 2: $r=p=2$. As in the proof of Proposition 7.19 (case 2), we have $\tilde{a} \equiv$ $\mathrm{V}^{n-1}\left(b t^{-1}+c t^{-2}\right) \bmod W_{n}\left(\mathcal{O}_{L}\right)$, with $b, c \in K_{0}$, and

$$
g_{0}^{2}-g_{0}=(\tilde{a}, 1-x t)_{L_{x}, \sigma_{0}}=V^{n-1}\left(b x+c x^{2}\right) .
$$

This implies $c=b^{2}$; hence $a \in H_{p^{n}}^{1}\left(\mathcal{O}_{L}\right)=\operatorname{fil}_{1} H_{p^{n}}^{1}(L)$.

Case 3: $1 \leq e \leq n-1$ and $r>2$. By Corollary 7.18(2) we have

$$
\tilde{a} \equiv \mathrm{V}^{n-1}\left(b_{j}[t]^{-(r-1)}\right)+\mathrm{V}^{n-e}\left(c_{j}[t]^{-r_{0} p}\right) \bmod \operatorname{fil}_{r-1}^{F} W_{n}(L),
$$

where $b_{j} \in K_{j}$ and $c_{j} \in W_{e}\left(K_{j}\right), j=0,1$. By Lemma 7.15(1) we have

$$
\left(\mathrm{V}^{n-1}\left(b_{j}[t]^{-(r-1)}\right), 1-x t^{r-1}\right)_{L_{x}, \sigma_{j}}=-(r-1) \mathrm{V}^{n-1}\left(b_{j} x\right) ;
$$

and by Lemma 7.15(2) we have

$$
\left(\mathrm{V}^{n-e}\left(c_{j}[t]^{-r_{0} p}\right), 1-x t^{r-1}\right)_{L_{x}, \sigma_{j}}=0
$$

Thus by (2),

$$
F\left(g_{j}\right)-g_{j}=\left(\tilde{a}, 1-x t^{r-1}\right)_{L_{x}, \sigma_{j}}=-(r-1) \mathrm{V}^{n-1}\left(b_{j} x\right)
$$


By Lemma 8.6 we have $F\left(g_{j}\right)-g_{j}=0$, for $j=0,1$. Hence $\tilde{a} \in \operatorname{fil}_{r-1}^{F} W_{n}(L)$, by Proposition 7.19.

Case 4: $e \geq n$ and $r>2$. By Corollary 7.18(3) we have

$$
a \equiv c_{j}[t]^{-p^{e-n+1} r_{0}}+V^{n-1}\left(b_{j}[t]^{-(r-1)}\right) \bmod \operatorname{fil}_{r-1}^{F} W_{n}(L),
$$

where $c_{j} \in W_{n}\left(K_{j}\right)$ and $b_{j} \in K_{j}$, for $j=0,1$. As in the third case, the following equality follows from Lemma 7.15 for $j=0,1$ :

$$
F\left(g_{j}\right)-g_{j}=\left(\tilde{a}, 1-x t^{r-1}\right)_{L_{x}, \sigma_{j}}=-(r-1) \mathrm{V}^{n-1}\left(b_{j} x\right) .
$$

Hence $\tilde{a} \in \mathrm{fil}_{r-1}^{F} W_{n}(L)$ as before. This completes the proof.

Theorem 8.8. Set $L \in \Phi$ and $r \geq 0$. Then

$$
\mathrm{fil}_{r} H^{1}(L)=\widetilde{H^{1}}\left(\mathcal{O}_{L}, \mathfrak{m}^{-r}\right)
$$

that is, the Artin conductor is motivic, Art $=c^{H^{1}}$. Furthermore, $\left(c^{H^{1}}\right)^{\leq 1}$ is a conductor of level 1.

Proof. The last statement follows from the first and Proposition 8.5. By Corollary 4.29 it suffices to show the corresponding statement on the subsheaf of $p^{n}$-torsion, for all $n \geq 1$. Here the proof is the same as in Theorem 7.20, if we replace $W_{n}$ everywhere by $H_{p^{n}}^{1}$, fil ${ }^{F}$ by fil, the reference to Proposition 7.5 by a reference to Proposition 8.5 and the reference to Proposition 7.19 by a reference to Proposition 8.7.

\subsection{Lisse sheaves of rank 1}

In this subsection we fix a prime number $\ell \neq p$, an algebraic closure $\overline{\mathbb{Q}}_{\ell}$ of $\mathbb{Q}_{\ell}$ and a compatible system of primitive roots of unity $\left\{\zeta_{n}\right\} \subset \overline{\mathbb{Q}}_{\ell}^{\times}$.

8.9. We denote by $\operatorname{Lisse}^{1}(X)$ the group of isomorphism classes of lisse $\overline{\mathbb{Q}}_{\ell}$-sheaves on $X$ of rank 1 , with group structure given by $\otimes$. Note that

$$
\operatorname{Lisse}^{1}(X) \cong \underset{E / \mathbb{Q}_{\ell}}{\lim } H_{\text {ét }}^{1}\left(X, \mathcal{O}_{E}^{\times}\right):=\underset{E / \mathbb{Q}_{\ell}}{\lim _{n}}{\underset{\lim }{n}}_{\text {ét }^{1}}\left(X,\left(\mathcal{O}_{E} / \mathfrak{m}_{E}^{n}\right)^{\times}\right),
$$

where $E$ runs over subextensions of $\overline{\mathbb{Q}}_{\ell} / \mathbb{Q}_{\ell}$ which are finite over $\mathbb{Q}_{\ell}$, and $\mathcal{O}_{E}$ and $\mathfrak{m}_{E}$ denote the ring of integers and the maximal ideal, respectively. Indeed, a sheaf $M \in \operatorname{Lisse}^{1}(X)$ corresponds uniquely to a continuous morphism $\pi_{1}^{\mathrm{ab}}(X) \rightarrow \overline{\mathbb{Q}}_{\ell}^{\times}$, which in particular implies that it factors as a continuous morphism $\pi_{1}^{\text {ab }}(X) \rightarrow E^{\times}$, with some $E$ as before (see, for example, $[11,1.1]$ ). Since any representation of a profinite group in a finite-dimensional $E$-vector space has an $\mathcal{O}_{E}$-lattice, we see that such a morphism factors via a continuous map

$$
\pi_{1}^{\mathrm{ab}}(X) \rightarrow \operatorname{Aut}_{\mathcal{O}_{E}}\left(\mathfrak{m}_{E}^{-j} \mathcal{O}_{E}\right)=\mathcal{O}_{E}^{\times}
$$


The isomorphism classes of such maps correspond uniquely to elements in $\lim _{n} H_{\text {ét }}^{1}$ $\left(X,\left(\mathcal{O}_{E} / \mathfrak{m}_{E}^{n}\right)^{\times}\right)$. By Section 8.1 and Lemma 8.2, the isomorphism (8.9.1) induces the structure of a Nisnevich sheaf with transfers on $X \mapsto \operatorname{Lisse}^{1}(X)$ - that is,

\section{Lisse $^{1} \in \mathbf{N S T}$.}

Write

$$
\left|\mathcal{O}_{E} / \mathfrak{m}_{E}\right|=\ell^{r_{E}}, \quad \ell^{r_{E}}-1=p^{s_{E}} \cdot h_{E}, \quad \text { with }\left(h_{E}, p\right)=1, s_{E} \geq 0 .
$$

Then $\mu_{\ell^{r} E-1}\left(\overline{\mathbb{Q}}_{\ell}\right) \subset \mathcal{O}_{E}^{\times}$and the roots of unity fixed at the beginning of this subsection induce a canonical isomorphism

$$
\mathcal{O}_{E}^{\times} \cong \mathbb{Z} / p^{s_{E}} \times \mathbb{Z} / h_{E} \times U_{E}^{(1)} .
$$

Since $U_{E}^{(1)}$ is a pro- $\ell$ group, this yields the decomposition

$$
\text { Lisse }^{1}=\text { Lisse }^{1, p^{\prime}} \oplus H_{p^{\infty}}^{1} \quad \text { in NST, }
$$

where

$$
\begin{gathered}
X \mapsto \operatorname{Lisse}^{1, p^{\prime}}(X):=\underset{E / \mathbb{Q}_{\ell}}{\lim _{n}} \underbrace{}_{n} H_{\text {ét }}^{1}\left(X, \mathbb{Z} / h_{E} \times U_{E}^{(1)} / U_{E}^{(n)}\right), \\
X \mapsto H_{p^{\infty}}^{1}(X):=\underset{E / \mathbb{Q}_{\ell}}{\lim } H_{\text {ét }}^{1}\left(X, \mathbb{Z} / p^{s_{E}}\right)=H_{p^{\infty}}^{1}(X) .
\end{gathered}
$$

Set $L \in \Phi$. For $j \geq 0$, we define

$$
\mathrm{fil}_{j} \operatorname{Lisse}^{1}(L):= \begin{cases}\operatorname{Im}\left(\operatorname{Lisse}^{1}\left(\mathcal{O}_{L}\right) \rightarrow \operatorname{Lisse}^{1}(L)\right) & \text { if } j=0 \\ \operatorname{Lisse}^{1, p^{\prime}}(L) \oplus \operatorname{fil}_{j} H_{p^{\infty}}^{1}(L) & \text { if } j \geq 1\end{cases}
$$

where $\operatorname{fil}_{j} H_{p^{\infty}}^{1}(L)=\cup_{n} \mathrm{fil}_{j} H_{p^{n}}^{1}(L)$ is defined in Section 8.4.

Corollary 8.10. Let the notation be as in Section 8.9. Then the following are true:

(1) Lisse $^{1} \in \mathbf{R S C}_{\mathrm{Nis}}$;

(2) the motivic conductor is given by

$$
c_{L}^{\mathrm{Lisse}^{1}}(M)=\min \left\{j \geq 0 \mid M \in \mathrm{fil}_{j} \operatorname{Lisse}^{1}(L)\right\},
$$

which furthermore restricts to a level 1 conductor;

(3) if $X \in \mathbf{S m}$ is proper over $k$ and $U \subset X$ is dense open, then

$$
h_{\mathbf{A}^{1}}^{0}\left(\operatorname{Lisse}^{1}\right)(U)=\operatorname{Lisse}^{1, p^{\prime}}(U) \oplus H_{p^{\infty}}^{1}(X)
$$

(see Section 4.30 for notation).

Proof. Note that Lisse ${ }^{1, p^{\prime}} \in H I_{\mathrm{Nis}}$. Hence (1) and (2) follow directly from Theorem 8.8 together with Corollary 4.29 and Lemma 4.20. For (3), observe that by Theorem 8.8 and the definition of the Artin conductor, we have $H_{p^{\infty}}^{1}\left(\mathcal{O}_{L}, \mathfrak{m}_{L}^{-1}\right)=H_{p^{\infty}}^{1}\left(\mathcal{O}_{L}\right)$; hence the statement follows from Corollary 4.33 . 
Remark 8.11. Set $U \in \mathbf{S m}$ and denote by $\pi_{1}^{\mathrm{ab}, \mathrm{t}}(U / k)$ the abelian tame fundamental group in the sense of $[31,7]$; it is a quotient of $\pi_{1}^{\mathrm{ab}}(U)$. Denote by $\operatorname{Tame}^{1}(U)$ the subgroup of Lisse ${ }^{1}(U)$ consisting of those lisse sheaves of rank 1 whose corresponding representation factors via $\pi_{1}^{\mathrm{ab}, \mathrm{t}}(U / k)$. Then

$$
h_{\mathbf{A}^{1}}^{0}\left(\operatorname{Lisse}^{1}\right)(U)=\operatorname{Tame}^{1}(U) .
$$

Indeed, we classically have $\operatorname{Tame}^{1}(C)=\operatorname{Lisse}^{1, p^{\prime}}(C) \oplus H_{p^{\infty}}^{1}(\bar{C})$, in the case where $C \in \mathbf{S m}$ is a curve over $k$ with smooth compactification $\bar{C}$. Hence this $\subset$ inclusion follows from Corollary 8.10(3) and the description of $\pi_{1}^{\mathrm{ab}, \mathrm{t}}(U / k)$ via curve-tameness [26]. The other inclusion follows from the $\mathbf{A}^{1}$-invariance of Tame ${ }^{1}$.

\section{Torsors under finite group schemes over a perfect field}

In this section, $k$ is a perfect field of positive characteristic $p$. We fix an algebraic closure $\bar{k}$ of $k$. The term $k$-group is short for commutative group scheme of finite type over $k$.

Lemma 9.1. Let $G$ be a finite $k$-group. Then there exists an exact sequence of sheaves on $(\mathrm{Sch} / k)_{\mathrm{fppf}}$, the fppf site on $k$-schemes,

$$
0 \rightarrow G \rightarrow H_{1} \rightarrow H_{2} \rightarrow 0
$$

with $H_{i}, i=1,2$, smooth $k$-groups. Furthermore, if we denote by $u:(\mathrm{Sch} / k)_{\mathrm{fppf}} \rightarrow$ $(\mathrm{Sch} / k)_{\text {ét }}$ the morphism from the fppf site to the étale site, then the sequence induces a canonical isomorphism

$$
R u_{*} G \cong\left[H_{1} \rightarrow H_{2}\right]
$$

in the derived category of abelian sheaves on $(\mathrm{Sch} / k)$ ét. In particular, for all $n \geq 0$ the presheaf on $\mathbf{S m}$

$$
\mathbf{S m} \ni X \mapsto H^{n}\left(X_{\mathrm{fppf}}, G\right) \cong H^{n}\left(X_{\text {ét }}, H_{1} \rightarrow H_{2}\right)
$$

admits the structure of a presheaf with transfers. This transfer structure does not depend on the choice of sequence (9.1.1) (up to isomorphism).

Proof. By a result of Raynaud (see [3, 3.1.1]), there exists a closed immersion $G \hookrightarrow A$, with $A$ an abelian variety. By [13, $\operatorname{Exp} V I_{A}$, Théorème 3.2], the fppf-quotient sheaf $(A / G)_{\text {fppf }}$ is representable by a $k$-group $A / G$ and the quotient map $A \rightarrow A / G$ is finite and faithfully flat. Hence $A / G$ is reduced and hence a smooth $k$-group. This shows the existence of a sequence (9.1.1). By [19, Théorème (11.7)], a smooth $k$-group is acyclic for the direct image functor

$$
u_{*}: \operatorname{Shv}\left((\operatorname{Sch} / k)_{\text {fppf }}\right) \rightarrow \operatorname{Shv}\left((\operatorname{Sch} / k)_{\text {ét }}\right) .
$$

Hence sequence (9.1.1) is a $u_{*}$-acyclic resolution of the fppf sheaf $G$, which yields the canonical isomorphism (9.1.2). Since $H_{1} \rightarrow H_{2}$ is a complex of étale sheaves with transfers, the presheaf (9.1.3) has transfers, by [39, Lemma 6.23]. Finally, we have to show that this transfer structure does not depend on the resolution (9.1.1). Assume $0 \rightarrow G \rightarrow L_{1} \rightarrow$ $L_{2} \rightarrow 0$ is a second such exact sequence. We obtain a commutative diagram with exact rows in $(\mathrm{Sch} / k)_{\mathrm{fppf}}$ : 


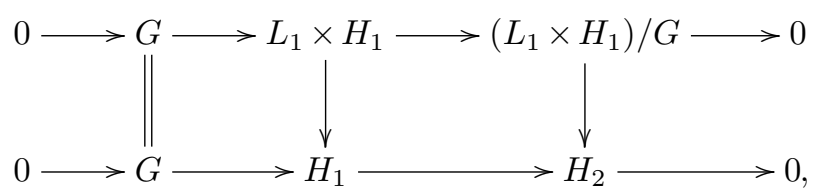

where the vertical arrows are induced by projection and the top horizontal arrow on the left is the diagonal embedding of $G$; we also have such a sequence with $H$ replaced by $L$ in the lower line. This yields the isomorphism $\left[H_{1} \rightarrow H_{2}\right] \cong\left[L_{1} \rightarrow L_{2}\right]$ in the derived category of étale sheaves with transfers, proving the final statement.

Notation 9.2. Let $G$ be a finite $k$-group. Then we denote by $H^{1}(G) \in \mathbf{P S T}$ the presheaf with transfers from Lemma 9.1,

$$
X \mapsto H^{1}(G)(X):=H^{1}\left(X_{\mathrm{fppf}}, G\right) .
$$

Lemma 9.3. Let $\operatorname{Gal}(\bar{k} / k)$ be the absolute Galois group of $k$ and $G$ an étale $k$-group. Then the functor

$$
\operatorname{Sm} \ni X \mapsto H^{n}\left(\operatorname{Gal}(\bar{k} / k), G\left(X_{\bar{k}}\right)\right)
$$

defined by the Galois cohomology groups is a proper sheaf in $\mathbf{R S C}_{\mathrm{Nis}}$ in the sense of Definition 4.26.

Proof. The composition

$$
\operatorname{Cor}_{k}(X, Y) \rightarrow \operatorname{Cor}_{\bar{k}}\left(X_{\bar{k}}, Y_{\bar{k}}\right) \rightarrow \operatorname{Hom}_{\mathbf{A b}}\left(G\left(Y_{\bar{k}}\right), G\left(X_{\bar{k}}\right)\right)
$$

factors through the homomorphism of Galois modules; hence formula (9.3.1) $\in$ PST. Since $G$ is étale, we have $G\left(X_{\bar{k}}\right)=G(\bar{k})^{\pi_{0}\left(X_{\bar{k}}\right)}$. It follows that formula (9.3.1) is $\mathbf{A}^{1}$-invariant and that restrictions to dense open subsets are isomorphisms. Hence it is a Nisnevich sheaf and proper.

Lemma 9.4. Let $G$ be an étale $k$-group. Then the exact sequence

$$
E(X): 0 \rightarrow H^{1}\left(\operatorname{Gal}(\bar{k} / k), G\left(X_{\bar{k}}\right)\right) \rightarrow H^{1}(G)(X) \rightarrow K^{1}(X) \rightarrow 0,
$$

with

$$
K^{1}(X):=\operatorname{Ker}\left(H^{1}\left(X_{\bar{k}, \text { ét }}, G_{\bar{k}}\right)^{\operatorname{Gal}(\bar{k} / k)} \rightarrow H^{2}\left(\operatorname{Gal}(\bar{k} / k), G\left(X_{\bar{k}}\right)\right)\right),
$$

coming from the $E_{2}$-page of the Hochschild-Serre spectral sequence defines an exact sequence $X \mapsto E(X)$ in PST.

Proof. First note that by Grothendieck's theorem (see Lemma 9.1) we have $H^{1}(G)(X)=$ $H^{1}\left(X_{\text {ét }}, G\right)$, so that the sequence $E(X)$ is indeed induced by the Hochschild-Serre spectral sequence. We show that transfers act on the whole spectral sequence. By a limit argument, it suffices to consider finite Galois extensions $L / k$ and the corresponding 
spectral sequence. Let $G \rightarrow I^{\bullet}$ be an injective resolution in $\mathrm{Sh}_{\text {ét }}\left(\mathbf{C o r}_{k}\right)$, the category of étale sheaves with transfers. Then

$$
H^{i}\left(X_{\text {ét }}, G\right)=H^{i}\left(I^{\bullet}(X)\right)=H^{i}\left(I^{\bullet}\left(X_{L}\right)^{\operatorname{Gal}(L / k)}\right), \quad i \geq 0,
$$

for all $X \in \mathbf{S m}$ (see [39, Lemma 6.23]). Moreover, $H^{i}\left(X_{L \text {, ét }}, I^{n}\right)=0=H^{i}\left(X_{\text {ét }}, I^{n}\right)$ for $i \geq 1$ and $n \geq 0$ (see again [40]). Hence

$$
H^{i}\left(\operatorname{Gal}(L / k), I^{n}\left(X_{L}\right)\right)=0 .
$$

Let $C^{\bullet}(\operatorname{Gal}(L / k), M)$ be the complex of cochains computing the cohomology of the $\operatorname{Gal}(L / k)$-module $M$. By equations (9.4.1) and (9.4.2), the cohomology groups $H_{\text {ét }}^{i}(X, G)$ are the cohomology groups of the total complex associated to the double complex $C^{\bullet}\left(\operatorname{Gal}(L / k), I^{\bullet}\left(X_{L}\right)\right)$. The Hochschild-Serre spectral sequence arises from a filtration of this complex. Furthermore, the canonical map $\operatorname{Cor}_{k}(X, Y) \times \operatorname{Gal}(L / k) \rightarrow \operatorname{Cor}_{k}\left(X_{L}, Y_{L}\right)$, $(\alpha, \sigma) \mapsto\left(\alpha \otimes_{k} L\right) \circ\left(\operatorname{id}_{X \times_{k} Y} \times \sigma\right)$ induces the structure of a complex of presheaves with $\operatorname{Gal}(L / k)$-equivariant transfers on $X \mapsto I^{\bullet}\left(X_{L}\right)$. Hence $X \mapsto C^{\bullet}\left(\operatorname{Gal}(L / k), I^{\bullet}\left(X_{L}\right)\right)$ is a double complex in PST. This proves the lemma.

Lemma 9.5. Let $G$ be an étale $k$-group of order prime to $p$. Then $H^{1}(G) \in \mathbf{H I}_{\mathrm{Nis}}$ (see Notation 9.2 for notation).

Proof. In this case $G_{\bar{k}}$ is a constant finite $k$-group of order prime to $p$. By [48, Cor 5.29], the presheaf $X \mapsto K^{1}(X)$ from Lemma 9.4 is $\mathbf{A}^{1}$-invariant, and by Lemmas 8.2 and 9.3 it is a Nisnevich sheaf. Thus the claim follows from Lemmas 9.4 and 9.3.

Lemma 9.6. Let $G$ be an étale $k$-group of p-primary order. Then $H^{1}(G) \in \mathbf{R S C}_{\mathrm{Nis}}$ and the motivic conductor $c^{H^{1}(G)}$ is given by

$$
c_{L}^{H^{1}(G)}: H^{1}(G)(L) \rightarrow \bigoplus_{i} H_{\text {ét }}^{1}\left(\operatorname{Spec} L_{i}, G_{\bar{k}}\right) \stackrel{\max _{i}\left\{c_{L_{i}}^{H^{1}\left(G_{\bar{k}}\right)}\right\}}{\longrightarrow} \mathbb{N}_{0},
$$

where $L \otimes_{k} \bar{k}=\prod_{i} L_{i}$ and $c^{H^{1}\left(G_{\bar{k}}\right)}$ is computed in Theorem 8.8 (note that $G_{\bar{k}}=\oplus_{j} \mathbb{Z} / p^{n_{j}}$ ). In particular, $\left(c^{H^{1}(G)}\right)^{\leq 1}$ is a conductor. Moreover, if $X$ is smooth proper and $U \subset X$ is dense open, then $h_{\mathbf{A}^{1}}^{0}\left(H^{1}(G)\right)(U)=H^{1}(G)(X)$ (see Section 4.30 for notation).

Proof. Note in this case that $H^{2}\left(\operatorname{Gal}(\bar{k} / k), G\left(X_{\bar{k}}\right)\right)=0$ (see, for example, [2, Exp X, Théorème 5.1]). Thus the first statement follows from Lemmas 9.4, 9.3, 8.2 and 4.27, Propositions 4.19 and 4.21 and Theorem 8.8. For the final statement, observe that

$$
\widetilde{H^{1}(G)}\left(\mathcal{O}_{L}, \mathfrak{m}_{L}^{-1}\right)=H^{1}(G)\left(\mathcal{O}_{L}\right)
$$

This follows directly from the explicit description of the motivic conductor on $H^{1}\left(G_{\bar{k}}\right)$ in Theorem 8.8. Hence the final statement follows from Corollary 4.33. 
Lemma 9.7. Let $G$ be an infinitesimal finite $k$-group. Then

$$
H^{1}\left(X_{\mathrm{fppf}}, G\right) \cong H^{1}\left(X_{\bar{k}, \mathrm{fpp}}, G_{\bar{k}}\right)^{\operatorname{Gal}(\bar{k} / k)}, \quad \text { for all } X \in \mathbf{S m} .
$$

Furthermore, this isomorphism induces an isomorphism in NST (compare Proposition 4.21 for notation):

$$
H^{1}(G) \cong\left(R_{\bar{k} / k} H^{1}\left(G_{\bar{k}}\right)\right)^{\operatorname{Gal}(\bar{k} / k)} .
$$

Proof. There is also a Hochschild-Serre spectral sequence for the fppf cohomology (see, for example, [40, III, Remark 2.21]). Since $G$ is infinitesimal, we have $G(Y)=0$ for all reduced schemes $\mathrm{Y}$ over $k$. Thus the fppf version of the exact sequence $E(X)$ from Lemma 9.4 yields the first isomorphism. By Lemma 9.1, this isomorphism is compatible with the transfer structure. It remains to show that $H^{1}(G)$ is a Nisnevich sheaf. By the remark from the beginning of this proof, any sequence (9.1.1) yields an injection $H_{1} \hookrightarrow H_{2}$ when restricted to Sm. Thus isomorphism (9.1.2) implies

$$
R u_{*} G \cong\left(H_{2} / H_{1}\right)_{\text {ét }}[-1]
$$

in the derived category of étale sheaves on $\mathbf{S m}$, where $\left(H_{2} / H_{1}\right)$ ét denotes the étale sheafification of the presheaf $X \mapsto H_{2}(X) / H_{1}(X)$. Hence

$$
H^{1}(G)(X)=H^{0}\left(X,\left(H_{2} / H_{1}\right)_{\text {ét }}\right) .
$$

It follows that $H^{1}(G)$ is even an étale sheaf.

Lemma 9.8. Assume $G$ is an infinitesimal finite $k$-group of multiplicative type. Then $H^{1}(G) \in \mathbf{H I}_{\mathrm{Nis}}$.

Proof. By Lemma 9.7 we may assume $k=\bar{k}$. In this case $G$ is diagonalisable and we find an exact sequence (9.1.1) with $H_{i}=\mathbf{G}_{m}^{n_{i}}$, some $n_{i} \geq 1$ (see [12, IV, $\S 1,1.5$ Corollary]). The statement follows from the $\mathbf{A}^{1}$-invariance of $X \mapsto H^{i}\left(X_{\mathrm{Zar}}, \mathbf{G}_{m}\right), i=0,1$, and Hilbert 90 .

9.9. We denote

$$
\alpha_{p}:=\operatorname{Ker}\left(F: \mathbf{G}_{a} \rightarrow \mathbf{G}_{a}\right),
$$

where $F$ is the absolute Frobenius on the additive group. Then $\alpha_{p}$ is a unipotent infinitesimal finite $k$-group. Set $L \in \Phi$ and let $t \in \mathcal{O}_{L}$ be a local parameter. Recall from Section 7.3 that $\operatorname{fil}_{j} \mathbf{G}_{a}(L):=\operatorname{fil}_{j} W_{1}(L)$ is given by

$$
\operatorname{fil}_{j} \mathbf{G}_{a}(L)= \begin{cases}\mathcal{O}_{L} & \text { if } j=0 \\ \frac{1}{t^{j-1}} \cdot \mathcal{O}_{L} & \text { if }(j, p)=1 \\ \frac{1}{t^{j}} \cdot \mathcal{O}_{L} & \text { if } p \mid j .\end{cases}
$$

We denote by

$$
\operatorname{fil}_{j} H^{1}\left(\alpha_{p}\right)(L)
$$


the image of $\operatorname{fil}_{j} \mathbf{G}_{a}(L)$ under the connecting homomorphism

$$
\delta: \mathbf{G}_{a}(L) \rightarrow H^{1}\left(\alpha_{p}\right)(L)=H^{1}\left(\operatorname{Spec} L_{\mathrm{fppf}}, \alpha_{p}\right) .
$$

Note that fil $_{j} H^{1}\left(\alpha_{p}\right)(L)$ is also equal to the image of the Frobenius saturated filtration $\mathrm{fil}_{j}^{F} W_{1}(L)$.

Proposition 9.10. We have $H^{1}\left(\alpha_{p}\right) \in \mathbf{R S C}_{\mathrm{Nis}}$, and the motivic conductor $c^{H^{1}\left(\alpha_{p}\right)}$ on $H^{1}\left(\alpha_{p}\right)$ is given by

$$
c_{L}^{H^{1}\left(\alpha_{p}\right)}(b)=\min \left\{j \geq 0 \mid b \in \operatorname{fil}_{j} H^{1}\left(\alpha_{p}\right)(L)\right\} .
$$

In particular, either $b \in H^{1}\left(\alpha_{p}\right)\left(\mathcal{O}_{L}\right)$ or $c_{L}^{H^{1}\left(\alpha_{p}\right)}(b) \geq 2$. Furthermore, it restricts to a level 2 conductor.

Proof. Denote the collection of maps $H^{1}\left(\alpha_{p}\right)(L) \rightarrow \mathbb{N}_{0}$ defined by the right-hand side of equation (9.10.1) by $c$. By Proposition 7.5 and Lemma 4.28, $c$ satisfies (c1)-(c6) except possibly for $(\mathrm{c} 4)$. (For (c5), note that $\mathbf{G}_{a}(Y) \rightarrow H^{1}\left(\alpha_{p}\right)(Y)$ is surjective for any affine scheme $Y$ over $k$.) We claim that $c^{\leq 2}$ satisfies (c4). Let $X$ be a smooth $k$-scheme and set $b \in H^{1}\left(\alpha_{p}\right)\left(\mathbf{A}_{X}^{1}\right)$ with

$$
c_{k(x)_{\infty}}\left(\rho_{x}^{*} b\right) \leq 1, \quad \text { for all } x \in X \text { with } \operatorname{trdeg}(k(x) / k) \leq 1,
$$

where $\rho_{x}: \operatorname{Spec} k(x)(t)_{\infty}=\operatorname{SpecFrac}\left(\mathcal{O}_{\mathbf{P}_{x}^{1}, \infty}^{h}\right) \rightarrow \mathbf{A}_{X}^{1}$ is the natural map. We want to show $b \in H^{1}\left(\alpha_{p}\right)(X)$. This is equivalent to $b=\pi^{*} i^{*} b$ in $H^{1}\left(\alpha_{p}\right)\left(\mathbf{A}_{X}^{1}\right)$; by the definition of $c$ and Lemma 9.7, we can therefore assume $k$ is algebraically closed. Furthermore, the question is local on $X$, hence we can assume $X=\operatorname{Spec} A$ affine. Note that for a general $\beta \in H^{1}\left(\alpha_{p}\right)(L) \backslash H^{1}\left(\alpha_{p}\right)\left(\mathcal{O}_{L}\right)$, we have $c_{L}(\beta) \geq 2$, as follows directly from equation (9.9.1). Hence condition (9.10.2) implies

$$
\rho_{x}^{*} b \in \operatorname{Im}\left(H^{1}\left(\alpha_{p}\right)\left(\mathcal{O}_{\mathbf{P}_{x}^{1}, \infty}^{h}\right) \rightarrow H^{1}\left(\alpha_{p}\right)\left(k(x)(t)_{\infty}\right)\right) .
$$

Denote by $b(x)$ the restriction of $b$ to $\mathbf{A}_{x}^{1}$. Since $H^{1}\left(\alpha_{p}\right)$ is a Nisnevich sheaf, we conclude

$$
b(x) \in H^{1}\left(\alpha_{p}\right)\left(\mathbf{P}_{x}^{1}\right)=H^{1}\left(\alpha_{p}\right)(x) .
$$

Thus we find a polynomial $\tilde{b}=b_{0}+b_{1} t+\cdots+b_{n} t^{n} \in A[t]$ mapping to $b$ such that for all points $x \in X$ with $\operatorname{trdeg}(k(x) / k) \leq 1$, there exist $c_{x} \in k(x)$ and $g_{x} \in k(x)[t]$ with

$$
\tilde{b}(x)=c_{x}+g_{x}^{p}, \quad \text { in } k(x)[t] .
$$

It follows immediately that $\tilde{b} \in A\left[t^{p}\right]$, and it remains to show $b_{i} \in A^{p}$ for all $i \geq 1$, since then $b=b_{0}$ in $H^{1}\left(\alpha_{p}\right)\left(\mathbf{A}_{X}^{1}\right)$. Thus we are reduced to showing the following: let $X=$ $\operatorname{Spec} A \rightarrow \mathbf{A}^{d}=\operatorname{Spec} k\left[x_{1}, \ldots, x_{d}\right]$ be an étale map and set $a \in A \backslash A^{p}$. Then there exists a smooth connected curve $i: C \hookrightarrow X$ such that $i^{*} a \in \mathcal{O}(C) \backslash \mathcal{O}(C)^{p}$. If $a \notin A^{p}$, we find a variable, say $x_{1}$, such that $a=a_{0}+a_{1} x_{1}+\cdots+a_{n} x_{1}^{n}$, where $a_{i} \in A^{p}\left[x_{2}, \ldots, x_{d}\right]:=B$ and $a \notin B\left[x_{1}^{p}\right]$. A tuple $\lambda=\left(\lambda_{2}, \ldots, \lambda_{d}\right) \in k^{d-1}$ induces a closed immersion $i_{\lambda}: \mathbf{A}^{1} \rightarrow \mathbf{A}^{d}$ given by $x_{1} \mapsto x_{1}, x_{i} \mapsto \lambda_{i}, i=2, \ldots, d$. Denote by $C_{\lambda}$ the pullback of $X$ along $i_{\lambda}$. Since $k$ is 
algebraically closed, we find a tuple $\lambda$ such that $a_{\mid C_{\lambda}} \notin \mathcal{O}\left(C_{\lambda}\right)^{p}$. This proves the claim; hence $c^{\leq 2}$ satisfies (c4).

Corollary 4.24 yields $c^{H^{1}\left(\alpha_{p}\right)} \leq c$. To show the other inequality it suffices by Corollary 4.40 to show the following: set $L \in \Phi$ and $t \in \mathcal{O}_{L}$ a local parameter, and let $\sigma: K \hookrightarrow \mathcal{O}_{L}$ be some coefficient field; extend it in the canonical way to $\sigma: K(x) \hookrightarrow \mathcal{O}_{L_{x}}$, where $L_{x}=\operatorname{Frac}\left(\mathcal{O}_{L}[x]_{(t)}^{h}\right)$. Assume $b \in \mathrm{fil}_{r} H^{1}\left(\alpha_{p}\right)(L), r \geq 1$. Then the following implication holds:

$$
\left(b, 1-x t^{r-1}\right)_{L_{x}, \sigma}=0 \quad \text { for all } \sigma \Rightarrow b \in \text { fil }_{r-1} H^{1}\left(\alpha_{p}\right)(L),
$$

where the local symbol on the left-hand side is the one from Section 4.37 for $H^{1}\left(\alpha_{p}\right)$ and $\sigma$ runs through all coefficient fields of $\mathcal{O}_{L}$. By (LS6), the local symbol on $H^{1}\left(\alpha_{p}\right)$ is given by

$$
\left(b, 1-x t^{r-1}\right)_{L_{x}, \sigma}=\delta\left(\operatorname{Res}_{t, \sigma}\left(\tilde{b} \operatorname{dlog}\left(1-x t^{r-1}\right)\right)\right),
$$

where $\tilde{b} \in \mathrm{fil}_{r} \mathbf{G}_{a}(L)$ is a lift of $b, \delta: \mathbf{G}_{a}(K(x)) \rightarrow H^{1}\left(\alpha_{p}\right)(K(x))$ is the connecting homomorphism and we use the isomorphism $L_{x}=K(x)((t))$ defined by $\sigma$ and $t$ to compute the residue symbol on the right. To prove implication (9.10.4), it suffices to consider $b$ modulo fil $r$. Fix $\sigma: K \hookrightarrow \mathcal{O}_{L}$.

Case 1: $(r, p)=1=(r-1, p)$. In this case, $\tilde{b} \equiv c / t^{r-1} \bmod \operatorname{fil}_{r-1} \mathbf{G}_{a}(L)$ for some $c \in K$. Hence

$$
\operatorname{Res}_{t, \sigma}\left(\tilde{b} \operatorname{dlog}\left(1-x t^{r-1}\right)\right)=-(r-1) c x .
$$

Since $\delta(-(r-1) c x)=0$ if and only if $c x \in K(x)^{p}$, this is only possible if $c=0$.

Case 2: $p \mid r-1$. In this case, $\mathrm{fil}_{r} H^{1}\left(\alpha_{p}\right)(L)=\mathrm{fil}_{r-1} H^{1}\left(\alpha_{p}\right)(L)$, and there is nothing to show.

Case 3: $p \mid r$. In this case, $\tilde{b} \equiv c / t^{r-1}+e / t^{r} \bmod f i l_{r-1} \mathbf{G}_{a}(L)$ for some $c, e \in K$. By the same argument as in the first case, we obtain the implication

$$
\left(b, 1-x t^{r-1}\right)_{L_{x}, \sigma}=0 \Rightarrow\left(\tilde{b}, 1-x t^{r-1}\right)_{L_{x}, \sigma}=0 \quad \text { in } \mathbf{G}_{a}(K(x)) .
$$

Since this hold for all $\sigma$, Proposition 7.19 (in the case where $n=1$ ) yields $\tilde{b} \in \operatorname{fil}_{r-1}^{F} \mathbf{G}_{a}(L)$, hence $b \in$ fil $_{r-1} H^{1}\left(\alpha_{p}\right)(L)$. This completes the proof.

Proposition 9.11. Let $G$ be a finite unipotent infinitesimal $k$-group. Then the following are true:

(1) $H^{1}(G) \in \mathbf{R S C}_{\mathrm{Nis}}$.

(2) The motivic conductor $c^{H^{1}(G)}$ restricts to a level 2 conductor.

(3) If $X$ is a proper smooth $k$-scheme and $U \subset X$ is open dense, then $h_{\mathbf{A}^{1}}^{0}\left(H^{1}(G)\right)(U)=$ $H^{1}(G)(X)$ (see Section 4.30 for notation).

Proof. (1). We find an exact sequence in the category of $k$-groups

$$
0 \rightarrow G \rightarrow H_{1} \rightarrow H_{2} \rightarrow 0
$$


with $H_{i}$ smooth unipotent $k$-groups. Indeed, by [12, V, $\left.\S 1,4.2,4.7\right]$, we find a closed immersion $G \hookrightarrow W_{n}^{N}:=H_{1}$ for some $n, N$, and by [12, IV $\left., \S 2,2.3\right]$ the quotient $H_{2}:=H_{1} / G$ is again unipotent, and it is automatically reduced, hence smooth. As in the proof of Lemma 9.7 , we find $H^{1}(G) \cong\left(H_{2} / H_{1}\right)$ ét, where $\left(H_{2} / H_{1}\right)$ ét is the étale sheaf associated to the presheaf $\mathbf{S m} \ni X \mapsto H_{2}(X) / H_{1}(X)$. Let $v: \mathbf{S m}_{\text {ét }} \rightarrow \mathbf{S m}_{\mathrm{Nis}}$ be the natural morphism of sites. Since $H_{1}$ is smooth unipotent, it is a successive extension of $\mathbf{G}_{a}$ s, hence $R^{1} v_{*} H_{1}=0$. We obtain an isomorphism in NST:

$$
H^{1}(G) \cong\left(H_{2} / H_{1}\right)_{\mathrm{Nis}}
$$

where $\left(H_{2} / H_{1}\right)_{\text {Nis }}$ is the Nisnevich sheaf associated to the presheaf $X \mapsto H_{2}(X) / H_{1}(X)$. Thus $H^{1}(G) \in \mathbf{R S C}_{\mathrm{Nis}}$ follows from $H_{i} \in \mathbf{R S C}_{\mathrm{Nis}}$ and [45, Theorem 0.1], which states that Nisnevich sheafification preserves SC-reciprocity.

(2). By [12, IV , 5.8], $G$ admits a descending sequence

$$
0=G_{n} \subset G_{n-1} \subset \cdots \subset G_{0}=G
$$

with successive quotients $G_{r-1} / G_{r} \cong \alpha_{p}$. In particular, $H^{2}\left(X_{\mathrm{fppf}}, G\right)=0$, for all affine smooth $k$-schemes $X$. Note that this induces for all $r \in[1, n]$ an exact sequence in NST

$$
0 \rightarrow H^{1}\left(G_{r}\right) \rightarrow H^{1}\left(G_{r-1}\right) \rightarrow H^{1}\left(\alpha_{p}\right) \rightarrow 0
$$

Indeed, by Lemma 9.7 this sequence is in NST; hence it suffices to check its exactness on any smooth affine $k$-scheme $X$, in which case it follows from $H^{0}\left(X_{\mathrm{fppf}}, \alpha_{p}\right)=0=$ $H^{2}\left(X_{\mathrm{fppf}}, G_{r}\right)$. By Proposition 9.10, the motivic conductor of $H^{1}\left(\alpha_{p}\right)$ restricts to a level 2 conductor, and by induction we may assume that so does the motivic conductor of $H^{1}\left(G_{r-1}\right)$. We deduce that the motivic conductor of $H^{1}\left(G_{r}\right)$ restricts to a level 2 conductor from sequence (9.11.2) and a similar argument as at the end of the proof of Proposition 8.5.

(3). We claim

$$
\widetilde{H^{1}(G)}\left(\mathcal{O}_{L}, \mathfrak{m}_{L}^{-1}\right)=H^{1}(G)\left(\mathcal{O}_{L}\right)
$$

The claim is true for $G=\alpha_{p}$, by the explicit formula of the motivic conductor in Proposition 9.10. Consider sequence (9.11.1) and assume the claim is proven for $G_{r}$. Set $b \in H^{1}\left(G_{r-1}\right)\left(\mathcal{O}_{L}, \mathfrak{m}^{-1}\right)$. By the exact sequence $(9.11 .2)$ and the claim for $\alpha_{p}$, we find a $c \in H^{1}\left(G_{r-1}\right)\left(\mathcal{O}_{L}\right)$ such that $b-c$ is in the image of $H^{1}\left(G_{r}\right)(L)$. By Proposition 4.19 we find

$$
b-c \in \widetilde{H^{1}\left(G_{r}\right)}\left(\mathcal{O}_{L}, \mathfrak{m}^{-1}\right)=H^{1}\left(G_{r}\right)\left(\mathcal{O}_{L}\right),
$$

which proves equation (9.11.3). Hence (3) follows from Corollary 4.33.

In summary:

Theorem 9.12. Let $G$ be a finite $k$-group. Then the following are true:

(1) $H^{1}(G) \in \mathbf{R S C}_{\mathrm{Nis}}$.

(2) The motivic conductor of $H^{1}(G)$ restricts to conductor of level 2, and if $G$ has no infinitesimal unipotent factor, to a conductor of level 1. 
(3) Write $G=G^{\prime} \times G_{\text {unip }}$ with $G_{\text {unip }}$ unipotent and $G^{\prime}$ without any unipotent subgroup, and let $X$ be smooth proper over $k$ and $U \subset X$ be dense open. Then

$$
h_{\mathbf{A}^{1}}^{0}\left(H^{1}(G)\right)(U)=H^{1}\left(G^{\prime}\right)(U) \oplus H^{1}\left(G_{\text {unip }}\right)(X) .
$$

Proof. By $[12, I V, \S 3,5.9]$ we can decompose $G$ uniquely into a product

$$
G=G_{e m} \times G_{e u} \times G_{i m} \times G_{i u},
$$

where $G_{e m}$ is étale multiplicative - that is, it is an étale $k$-group without $p$-torsion; $G_{e u}$ is étale unipotent - that is, it is an étale $k$-group with $p$-primary torsion; $G_{i m}$ is infinitesimal and of multiplicative type; and $G_{i u}$ is an infinitesimal unipotent $k$-group. Hence the statement follows from Lemmas 9.5, 9.6 and 9.8 and Proposition 9.11.

Remark 9.13. Let $G$ be a finite unipotent $k$-group. Note that by Theorem $9.12(3)$, the functor $X \mapsto H^{1}\left(X_{\mathrm{fppf}}, G\right)$ is a birational invariant for smooth proper $k$-schemes. This gives a new proof of this (probably) well-known result (it also follows, for example, from $[6])$.

Acknowledgments We thank the referee for helpful remarks. The first author is supported by DFG Heisenberg Grant RU 1412/2-2. Part of the work was done while he was a visiting professor at the TU München. He thanks Eva Viehmann for the invitation and the support. The second author is supported by JSPS KAKENHI Grant 15H03606 and the DFG SFB/CRC 1085 "Higher Invariants". The authors declare no conflicts of interest.

\section{References}

[1] M. Artin, A. Grothendieck And J. L. Verdier, Séminaire de géométrie algébrique du Bois-Marie 1963-1964. Théorie des topos et cohomologie étale des schémas. (SGA 4). Un séminaire dirigé par M. Artin, A. Grothendieck, J. L. Verdier. Avec la collaboration de N. Bourbaki, P. Deligne, B. Saint-Donat. Tome 1: Théorie des topos. Exposés I à IV, 2nd ed., 269 (Springer, Cham, Switzerland, 1972).

[2] M. Artin, A. Grothendieck And J. L. Verdier, Séminaire de géométrie algébrique du Bois-Marie 1963-1964. Théorie des topos et cohomologie étale des schémas (SGA 4). Un séminaire dirigé par M. Artin, A. Grothendieck, J. L. Verdier. Avec la collaboration de P. Deligne, B. Saint-Donat. Tome 3. Exposés IX à XIX, 305 (Springer, Cham, Switzerland, 1973).

[3] P. Berthelot, L. Breen and W. Messing, Théorie de Dieudonné cristalline. II, Lecture Notes in Mathematics, 930 (Springer-Verlag, Berlin, 1982).

[4] N. Bourbaki, Éléments de mathématique: Algèbre commutative. Chapitres 8 et 9 (Springer, Berlin, 2006). Reprint of the 1983 original.

[5] J.-L. BRYLinski, Théorie du corps de classes de Kato et revêtements abéliens de surfaces, Ann. Inst. Fourier (Grenoble) 33(3) (1983), 23-38.

[6] A. Chatzistamatiou and K. Rülling, Higher direct images of the structure sheaf in positive characteristic, Algebra Number Theory 5(6) (2011), 693-775.

[7] A. Chatzistamatiou and K. Rülling, Hodge-Witt cohomology and Witt-rational singularities, Doc. Math. 17 (2012), 663-781. 
[8] J.-L. Colliot-ThÉlène, R. T. Hoobler and B. Kahn, The Bloch-Ogus-Gabber theorem, in Algebraic K-theory (Toronto, ON, 1996), Fields Institute Communications, 16, pp. 31-94 (American Mathematical Society, Providence, RI, 1997).

[9] V. Costeanu, On the 2-typical de Rham-Witt complex, Doc. Math. 13 (2008), 413-452.

[10] P. Deligne, Équations différentielles à points singuliers réguliers, Lecture Notes in Mathematics, 163 (Springer-Verlag, Berlin, 1970).

[11] P. Deligne, La conjecture de Weil. II, Publ. Math. Inst. Hautes Études Sci. 52 (1980), $137-252$.

[12] M. Demazure and P. Gabriel, Groupes algébriques. Tome I: Géométrie algébrique, généralités, groupes commutatifs (Masson \& Cie, Paris, 1970). With an appendix by M. Hazewinkel).

[13] M. Demazure and A. Grothendieck (eds.), Schémas en groupes. I: Propriétés générales des schémas en groupes. Exposés I à VIIb. Séminaire de Géométrie Algébrique 1962/64, dirigé par Michel Demazure et Alexander Grothendieck, revised reprint,151 (Springer, Cham, Switzerland, 1970).

[14] T. Geisser and L. Hesselholt, On the $K$-theory of complete regular local $F_{p}$-algebras, Topology 45(3) (2006), 475-493.

[15] M. Gros, Classes de Chern et classes de cycles en cohomologie de Hodge-Witt logarithmique, Mém. Soc. Math. Fr. (N.S.) 21 (1985), 1-87.

[16] A. Grothendieck, Éléments de géométrie algébrique. IV. Étude locale des schémas et des morphismes de schémas. I, Publ. Math. Inst. Hautes Études Sci. 20 (1964), 5-259.

[17] A. Grothendieck, Éléments de géométrie algébrique. IV. Étude locale des schémas et des morphismes de schémas. II, Publ. Math. Inst. Hautes Études Sci. 24 (1965), 5-231.

[18] A. Grothendieck, Éléments de géométrie algébrique. IV. Étude locale des schémas et des morphismes de schémas. III, Publ. Math. Inst. Hautes Études Sci. 28 (1966), 5-255.

[19] A. Grothendieck, Le groupe de Brauer. III. Exemples et compléments, in Dix exposés sur la cohomologie des schémas, Advanced Studies in Pure Mathematics, 3, pp. 88-188 (North-Holland, Amsterdam, 1968).

[20] R. Hartshorne, On the De Rham cohomology of algebraic varieties, Publ. Math. Inst. Hautes Études Sci. 45 (1975), 5-99.

[21] L. Hesselholt and I . Madsen, On the De Rham-Witt complex in mixed characteristic, Ann. Sci. Éc. Norm. Supér. (4) 37(1) (2004), 1-43.

[22] L. Illusie, Complexe de de Rham-Witt et cohomologie cristalline, Ann. Sci. Éc. Norm. Supér. (4) 12(4) (1979), 501-661.

[23] F. Ivorra AND K. RüLling, $K$-groups of reciprocity functors, J. Algebraic Geom. 26(2) (2017), 199-278.

[24] B. Kahn, H. Miyazaki, S. Saito and T. Yamazaki, Motives with modulus, I: Modulus sheaves with transfers for non-proper modulus pairs, Épijournal Géom. Algébrique 5 (2021), https://epiga.episciences.org/7114.

[25] B. Kahn, H. Miyazaki, S. Saito and T. Yamazaki, Motives with modulus, II: Modulus sheaves with transfers for proper modulus pairs, Épijournal Géom. Algébrique 5 (2021) https://epiga.episciences.org/7115.

[26] B. Kahn, S. Saito and T. Yamazaki, Reciprocity sheaves, Compos. Math. 152(9) (2016), 1851-1898. With two appendices by K. Rülling.

[27] B. Kahn, S. Saito And T. Yamazaki, Reciprocity sheaves, II, to appear in Homology, Homotopy and Applications https://arxiv.org/abs/1707.07398.

[28] B. Kahn and T. Yamazaki, Voevodsky's motives and Weil reciprocity, Duke Math. J. 162(14) (2013), 2751-2796.

[29] K. Kato, A generalization of local class field theory by using K-groups. II, J. Fac. Sci. Univ. Tokyo Sect. IA Math. 27(3) (1980), 603-683. 
[30] K. Kato, Swan conductors for characters of degree one in the imperfect residue field case, in Algebraic K-theory and algebraic number theory (Honolulu, HI, 1987), Contemporary Mathematics, 83, pp. 101-131 (American Mathematical Society, Providence, RI, 1989).

[31] K. Kato, Class field theory, $\mathcal{D}$-modules, and ramification on higher-dimensional schemes. I, Amer. J. Math. 116(4) (1994), 757-784.

[32] K. Kato And H. Russell, Modulus of a rational map into a commutative algebraic group, Kyoto J. Math. 50(3) (2010), 607-622.

[33] K. Kato and H. Russell, Albanese varieties with modulus and Hodge theory, Ann. Inst. Fourier (Grenoble) 62(2) (2012), 783-806.

[34] M. Kerz And A. Schmidt, On different notions of tameness in arithmetic geometry, Math. Ann. 346(3) (2010), 641-668.

[35] M. Kerz And S. SAito, Chow group of 0-cycles with modulus and higher-dimensional class field theory, Duke Math. J. 165(15) (2016), 2811-2897.

[36] A. Langer And T. Zink, De Rham-Witt cohomology for a proper and smooth morphism, J. Inst. Math. Jussieu 3(2) (2004), 231-314.

[37] S. Matsuda, On the Swan conductor in positive characteristic, Amer. J. Math. 119(4) (1997), 705-739.

[38] H. Matsumura, Commutative Ring Theory, Cambridge Studies in Advanced Mathematics, 8, 2nd ed. (Cambridge University Press, Cambridge, UK, 1989).

[39] C. Mazza, V. Voevodsky and C. Weibel, Lecture Notes on Motivic Cohomology, Clay Mathematics Monographs, 2 (American Mathematical Society, Providence, RI, 2006).

[40] J. S. Milne, Étale Cohomology, Princeton Mathematical Series, 33 (Princeton University Press, Princeton, 1980).

[41] D. Popescu, General Néron desingularization and approximation, Nagoya Math. J. 104 (1986), 85-115.

[42] M. Raynaud, Anneaux locaux henséliens, Lecture Notes in Mathematics, 169 (SpringerVerlag, Berlin, 1970).

[43] K. RÜlling, The generalized de Rham-Witt complex over a field is a complex of zerocycles, J. Algebraic Geom. 16(1) (2007), 109-169.

[44] K. RüLLING, Erratum to: "The generalized de Rham-Witt complex over a field is a complex of zero-cycles" [J. Algebraic Geom. 16 (2007), no. 1, 109-169; mr2257322], J. Algebraic Geom. 16(4) (2007), 793-795.

[45] S. Saito, Purity of reciprocity sheaves, Adv. Math. 366 (2020), 107067.

[46] J.-P. Serre, Algèbre locale. Multiplicités, Cours au Collège de France, 1957-1958, rédigé par Pierre Gabriel, Lecture Notes in Mathematics, 11, 2nd ed. (Springer-Verlag, Berlin, 1965).

[47] J.-P. Serre, Groupes algébriques et corps de classes, Publications de l'Institut Mathématique de l'Université de Nancago, 7, 2nd ed. (Hermann, Paris, 1984).

[48] V. Voevodsky, Cohomological theory of presheaves with transfers, in Cycles, transfers, and motivic homology theories, Annals of Mathematics Studies, 143, pp. 87-137 (Princeton University Press, Princeton, 2000).

[49] V. Voevodsky, Triangulated categories of motives over a field, in Cycles, transfers, and motivic homology theories, Annals of Mathematics Studies, 143, pp. 188-238 (Princeton University Press, Princeton, 2000). 